\title{
HYPERPLANE ARRANGEMENTS AND MILNOR FIBRATIONS
}

\author{
by
}

Alexander I. Suciu

\begin{abstract}
There are several topological spaces associated to a complex hyperplane arrangement: the complement and its boundary manifold, as well as the Milnor fiber and its own boundary. All these spaces are related in various ways, primarily by a set of interlocking fibrations. We use cohomology with coefficients in rank 1 local systems on the complement of the arrangement to gain information on the homology of the other three spaces, and on the monodromy operators of the various fibrations.

Résumé (Arrangements d'hyperplans et fibrés de Milnor). — Étant donné un arrangement d'hyperplans, il y a plusieurs espaces topologiques qu'on puisse lui associer: le complémentaire et sa variété de bord, ainsi que la fibre de Milnor et son bord. Tous ces espaces sont reliés, en premier lieu par des fibrations. On utilise la cohomologie avec coefficients dans les systèmes locaux de rang 1 sur le complémentaire d'un arrangement d'hyperplans pour étudier l'homologie des trois autres espaces, et les opérateurs de monodromie des fibrations associées.
\end{abstract}

\section{Contents}

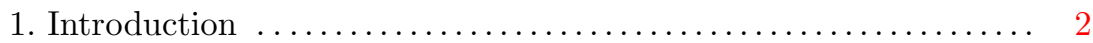

2. The complement of an arrangement. I. ................ 5

3. The complement of an arrangement. II. ................. 14

4. The Milnor fibration of an arrangement. I. . . . . . . . . . . . 20

5. The Milnor fibration of an arrangement. II. .............. 25

6. The boundary manifold of an arrangement $\ldots \ldots \ldots \ldots \ldots . \ldots 31$

7. The boundary of the Milnor fiber of an arrangement $\ldots \ldots \ldots 36$

A. Cohomology jumping loci $\ldots \ldots \ldots \ldots \ldots \ldots \ldots \ldots \ldots \ldots \ldots \ldots$

B. Finite, regular abelian covers $\ldots \ldots \ldots \ldots \ldots \ldots \ldots \ldots \ldots \ldots \ldots \ldots$

2000 Mathematics Subject Classification. - Primary 32S55, 57M10 Secondary 05B35, 14F35, $32 \mathrm{~S} 22,55 \mathrm{~N} 25$.

Key words and phrases. - Hyperplane arrangement, Milnor fibration, boundary manifold, graph manifold, characteristic variety, resonance variety, multinet, Alexander polynomial, formality.

Partially supported by NSF grant DMS-1010298. 


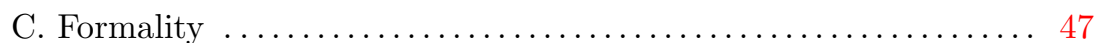

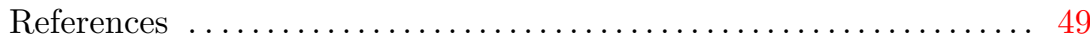

\section{Introduction}

1.1. Arrangements of hyperplanes. - This paper is mostly an expository survey, centered on the topology of complements of hyperplane arrangements, their Milnor fibrations, and their boundary structures. The presentation is loosely based on a set of notes for a mini-course given at the conference "Arrangements in Pyrénées", held in Pau, France in June 2012. Although we expanded the scope of those notes, and provided many more details and explanations, we made every effort to maintain the original spirit of the lectures, which was to give a brisk, self-contained introduction to the subject, and provide motivation for further study.

An arrangement is a finite collection of hyperplanes in a finite-dimensional, complex vector space. There are various ways to understand the topology of such an object. In this paper, we describe several topological spaces associated to a hyperplane arrangement, all connected to each other by means of inclusions, bundle maps, or covering projections. Associated to these spaces, there is a plethora of topological invariants of an algebraic nature: fundamental group and lower central series, Betti numbers and torsion coefficients, cohomology ring and Massey products, characteristic and resonance varieties, and so on. One of the main goals of the subject is to decide whether a given invariant is combinatorially determined, and, if so, to express it explicitly in terms of the intersection lattice of the arrangement.

As the title indicates, the focus of the paper is on the Milnor fibration of the complement of a hyperplane arrangement. We use cohomology with coefficients in rank 1 local systems on the complement to compute the homology groups of the Milnor fiber, with coefficients in a field not dividing the number of hyperplanes, and determine the characteristic polynomial of the monodromy operator acting on these homology groups. In the process, we also show how to compute various homological invariants of the boundary of the complement and the boundary of the Milnor fiber.

1.2. The complement. - Given an arrangement $\mathcal{A}$ in $\mathbb{C}^{d+1}$, the most direct approach is to study the complement, $M=\mathbb{C}^{d+1} \backslash \bigcup_{H \in \mathcal{A}} H$. The fundamental group of this space can be computed algorithmically, using the braid monodromy associated to a generic plane section. The cohomology ring of the complement was computed by Brieskorn in [3]. His result shows that $M$ is a formal space; in particular, all rational Massey products vanish. In [48], Orlik and Solomon gave a simple combinatorial description of the algebra $H^{*}(M, \mathbb{Z})$ : it is the quotient of the exterior algebra on classes dual to the meridians, modulo a certain ideal determined by the intersection lattice, $L(\mathcal{A})$. A comprehensive treatment of this topic can be found in [49].

Starting in the mid-to-late 1990s, the subject underwent a considerable shift of emphasis towards the study of the cohomology jump loci of the complement. These loci come in two basic flavors: the characteristic varieties, which are the jump loci for cohomology with coefficients in complex, rank one local systems, and the resonance 
varieties, which are the jump loci for the homology of the cochain complexes arising from multiplication classes in $H^{1}(M, \mathbb{C})$.

Since the complement of the arrangement is a smooth, quasi-projective variety, its characteristic varieties are finite unions of torsion-translates of algebraic subtori of the character group $\operatorname{Hom}\left(\pi_{1}(M), \mathbb{C}^{*}\right)$. Since the complement is a formal space, the resonance varieties of $M$ coincide with the tangent cone at the origin to the corresponding characteristic varieties, and thus are finite unions of rationally defined linear subspaces of $H^{1}(M, \mathbb{C})$.

For the degree- 1 resonance varieties, these subspaces were described combinatorially by Libgober and Yuzvinsky [40], and later by Falk and Yuzvinsky [25], solely in terms of multinets on sub-arrangements of $\mathcal{A}$. In general, though, the degree-1 characteristic varieties of an arrangement may contain components which do not pass through the origin, and it is still an open problem whether such components are combinatorially determined.

The characteristic and resonance varieties of an arrangement complement may also be defined for (algebraically closed) fields of positive characteristic. The nature of these varieties is less predictable, though. For instance, as noted in [43, 58, 24], both the tangent cone formula and the linearity of the resonance components fail in this setting. Furthermore, as shown in [42], non-vanishing Massey triple products may appear in positive characteristic.

1.3. The Milnor fibration. - A more refined topological invariant of an arrangement $\mathcal{A}$ is the Milnor fibration of its complement. For each hyperplane $H \in \mathcal{A}$, choose a linear form $f_{H}$ whose kernel is $H$. The polynomial $Q=\prod_{H \in \mathcal{A}} f_{H}$, then, is homogeneous of degree $n=|\mathcal{A}|$. As shown by Milnor [45] in a more general context, the restriction $Q: M \rightarrow \mathbb{C}^{*}$ is a smooth fibration. The typical fiber, $F=Q^{-1}(1)$, is called the Milnor fiber of the arrangement. We give a topological description of this fibration, building on previous joint work with Cohen and Denham $[8,6,14]$.

It turns out that $F$ is a regular, cyclic $n$-fold cover of the projectivized complement, $U=\mathbb{P}(M)$. The classifying homomorphism for this cover, $\delta: \pi_{1}(U) \rightarrow \mathbb{Z}_{n}$, takes each meridian generator to 1 . Embedding $\mathbb{Z}_{n}$ into $\mathbb{C}^{*}$ by sending 1 to a primitive $n$-th root of unity, we may view $\delta$ as a character on $\pi_{1}(U)$. The relative position of this character with respect to the characteristic varieties of $U$ determines the Betti numbers of the Milnor fiber $F$, as well as the characteristic polynomial of the algebraic monodromy.

More generally, given multiplicities $m_{H} \geq 1$ for each hyperplane $H \in A$, we consider the Milnor fibration of the multi-arrangement $(\mathcal{A}, m)$, defined by the homogeneous polynomial $Q(\mathcal{A}, m)=\prod_{H \in \mathcal{A}} f_{H}^{m_{H}}$. The Milnor fiber of this polynomial is now a regular, $N$-fold cyclic cover of the complement, where $N$ is the sum of the multiplicities. Not too surprisingly, the Betti numbers and other topological invariants of the Milnor fiber $F(\mathcal{A}, m)$ vary with the choice of $m$.

Although the complement of an arrangement and its Milnor fiber share some common features (for instance, they are both smooth, quasi-projective varieties), there are some striking differences between the two. For one, the homology groups of the Milnor fiber need not be torsion-free, as recent examples from [14] show. For another, the Milnor fiber may have non-vanishing Massey products, and thus be non-formal, 
as examples from [68] show. Finally, as noted in [19], the cohomology jump loci of the Milnor fiber may differ from those of the complement. All these novel qualitative features are related to the nature of multinets supported by the arrangement in question, or one of its sub-arrangements.

1.4. Boundary structures. - Both the projectivized complement and the Milnor fiber of an arrangement are non-compact manifolds (without boundary). Replacing these spaces by their compact versions allows us to study the behavior of the complement and the Milnor fibration as we approach the boundary, thereby revealing otherwise hidden phenomena.

Cutting off a regular neighborhood of the arrangement $\mathcal{A}$ yields a compact manifold with boundary, $\bar{U}$, onto which $U$ deform-retracts. The boundary manifold of the arrangement, then, is the smooth, compact, orientable $(2 d-1)$-dimensional manifold $\partial \bar{U}$. The homology groups are torsion-free, and the $\operatorname{ring} H^{*}(\partial \bar{U}, \mathbb{Z})$ is functorially determined by $H^{*}(U, \mathbb{Z})$, via a "doubling" construction.

Especially interesting is the case $d=2$, when the manifold $\partial \bar{U}$ is a graph-manifold, in the sense of Waldhausen. Following the approach from [11, 12], we describe the fundamental group, the cohomology ring, and the cohomology jump loci of $\partial \bar{U}$ in terms of the underlying graph structure, which in turn can be read off the intersection lattice of $\mathcal{A}$. Yet significant differences with the complement exist. For one, the boundary manifold is never formal, unless $\mathcal{A}$ is a pencil or a near-pencil. For another, the resonance varieties of $\partial \bar{U}$ may have non-linear components.

Intersecting now the Milnor fiber with a ball in $\mathbb{C}^{d+1}$ centered at the origin yields a compact manifold with boundary, $\bar{F}$, onto which $F$ deform-retracts. The boundary of the Milnor fiber, then, is the smooth, compact, orientable $(2 d-1)$-dimensional manifold $\partial \bar{F}$. We observe here that $\partial \bar{F}$ is a regular, cyclic $n$-fold cover of $\partial \bar{U}$, where $n=|\mathcal{A}|$, and identify a classifying homomorphism for this cover.

When $d=2$, the boundary of $\bar{F}$ is a 3 -dimensional graph-manifold. Using work of Némethi and Szilárd [46], we give a formula for the characteristic polynomial of the monodromy operator acting on $H_{1}(\partial \bar{F}, \mathbb{C})$, purely in terms of the Möbius function of $L_{\leq 2}(\mathcal{A})$. We also show that the manifold $\partial \bar{F}$ is never formal, unless $\mathcal{A}$ is a pencil or a near-pencil, and point out that $H_{1}(\partial \bar{F}, \mathbb{Z})$ typically has non-trivial $n$-torsion.

1.5. Organization of the paper. - The paper is divided roughly into three parts, following the approach outlined so far in this introduction.

The first part deals with the complement of a hyperplane arrangement $\mathcal{A}$. In $\S 2$, we discuss the combinatorics of $\mathcal{A}$, as it relates to the cohomology ring and resonance varieties of the complement, with special emphasis on multinets. In $\S 3$, we study the topology of $M(\mathcal{A})$, as reflected in the fundamental group and characteristic varieties, with special emphasis on orbifold fibrations and translated subtori in those varieties.

The second part covers the Milnor fibration defined by a multi-arrangement. In $\S 4$ we set up in all detail the regular covers into which the corresponding Milnor fiber fits. In $\S 5$, we study the homology of the Milnor fiber and the monodromy action, and discuss the formality properties and the cohomology jump loci of the Milnor fiber. 
The third part deals with the boundary structures associated to an arrangement. In $\S 6$ we discuss the boundary manifold of an arrangement, while in $\S 7$ we discuss the boundary of the Milnor fiber.

Sprinkled throughout the text there are about two dozen open problems. Most of these problems have been raised before; some are well-known, but some appear here for the first time.

We collect the necessary background material in three appendices at the end. Appendix A serves as a quick introduction to the cohomology jump loci of a space, and some of their properties. Appendix B deals with the homology groups and jump loci of finite, regular abelian covers of a space. Finally, Appendix C discusses formality properties of spaces, especially as they pertain to finite covers and jump loci.

1.6. Acknowledgements. - I wish to thank the organizers of the Arrangements in Pyrénées conference for giving me the opportunity to lecture in such a wonderful setting, and for their support and hospitality. I also wish to thank the University of Sydney for its support and hospitality while most of this paper was written up.

\section{The complement of an arrangement. I.}

In this section we describe the cohomology ring and the resonance varieties of the complement of a complex hyperplane arrangement.

2.1. Hyperplane arrangements. - An arrangement of hyperplanes is a finite set $\mathcal{A}$ of codimension- 1 linear subspaces in a finite-dimensional, complex vector space $\mathbb{C}^{d+1}$. Throughout, we will assume that the arrangement is central, that is, all the hyperplanes pass through the origin. The combinatorics of the arrangement is encoded in its intersection lattice, $L(\mathcal{A})$; this is the poset of all intersections of $\mathcal{A}$, ordered by reverse inclusion, and ranked by codimension. The arrangement is said to be essential if the intersection of all flats in $L(\mathcal{A})$ is $\{0\}$.

For each hyperplane $H \in \mathcal{A}$, let $f_{H}: \mathbb{C}^{d+1} \rightarrow \mathbb{C}$ be a linear form with kernel $H$. The product

$$
Q(\mathcal{A})=\prod_{H \in \mathcal{A}} f_{H}
$$

then, is a defining polynomial for the arrangement, unique up to a (non-zero) constant factor. Notice that $Q(\mathcal{A})$ is a homogeneous polynomial of degree equal to $|\mathcal{A}|$, the cardinality of the set $\mathcal{A}$.

On occasion, we will allow multiplicities on the hyperplanes. A multi-arrangement is a pair $(\mathcal{A}, m)$, where $\mathcal{A}$ is a hyperplane arrangement, and $m: \mathcal{A} \rightarrow \mathbb{Z}$ is a function with $m_{H} \geq 1$ for each $H \in \mathcal{A}$. We may also assign multiplicities to subspaces $X \in L(\mathcal{A})$, by letting $m_{X}=\sum_{H \leq X} m_{H}$. A defining polynomial for the multiarrangement $(\mathcal{A}, m)$ is the homogeneous polynomial

$$
Q_{m}(\mathcal{A})=\prod_{H \in \mathcal{A}} f_{H}^{m_{H}}
$$


2.2. The complement. - The main topological invariant associated to an arrangement $\mathcal{A}$ is its complement, $M(\mathcal{A})=\mathbb{C}^{d+1} \backslash \bigcup_{H \in \mathcal{A}} H$. This is a smooth, quasiprojective variety, with the homotopy type of a connected, finite $\mathrm{CW}$-complex of dimension $d+1$.

Example 2.1. - The Boolean arrangement $\mathcal{B}_{n}$ consists of the coordinate hyperplanes $H_{i}=\left\{z_{i}=0\right\}$ in $\mathbb{C}^{n}$. The intersection lattice is the Boolean lattice of subsets of $\{0,1\}^{n}$, ordered by reverse inclusion, while the complement is the complex algebraic torus $\left(\mathbb{C}^{*}\right)^{n}$.

Example 2.2. - The reflection arrangement of type $\mathrm{A}_{n-1}$, also known as the braid arrangement, consists of the diagonal hyperplanes $H_{i j}=\left\{z_{i}-z_{j}=0\right\}$ in $\mathbb{C}^{n}$. The intersection lattice is the lattice of partitions of $[n]=\{1, \ldots, n\}$, ordered by refinement. The complement is the configuration space $F(\mathbb{C}, n)$ of $n$ ordered points in $\mathbb{C}$. In the early 1960s, Fox and Neuwirth showed that $\pi_{1}(F(\mathbb{C}, n))=P_{n}$, the pure braid group on $n$ strings, while Neuwirth and Fadell showed that $F(\mathbb{C}, n)$ is aspherical.

The group $\mathbb{C}^{*}$ acts freely on $\mathbb{C}^{d+1} \backslash\{0\}$ via $\zeta \cdot\left(z_{0}, \ldots, z_{d}\right)=\left(\zeta z_{0}, \ldots, \zeta z_{d}\right)$. The orbit space is the complex projective space of dimension $d$, while the orbit map, $\pi: \mathbb{C}^{d+1} \backslash\{0\} \rightarrow \mathbb{C P}^{d}, z \mapsto[z]$, is the Hopf fibration. The set $\mathbb{P}(\mathcal{A})=\{\pi(H): H \in \mathcal{A}\}$ is an arrangement of codimension 1 projective subspaces in $\mathbb{C P}^{d}$; its complement, $U(\mathcal{A})$, coincides with the quotient $\mathbb{P}(M(\mathcal{A}))=M(\mathcal{A}) / \mathbb{C}^{*}$.

The Hopf map restricts to a bundle map, $\pi(\mathcal{A}): M(\mathcal{A}) \rightarrow U(\mathcal{A})$, with fiber $\mathbb{C}^{*}$. Fixing a hyperplane $H \in \mathcal{A}$, we see that $\pi(\mathcal{A})$ is also the restriction of the bundle map $\pi: \mathbb{C}^{d+1} \backslash H \rightarrow \mathbb{C P}^{d} \backslash \pi(H) \cong \mathbb{C}^{d}$. Clearly, this latter bundle is trivial; hence, we have a diffeomorphism

$$
M(\mathcal{A}) \cong U(\mathcal{A}) \times \mathbb{C}^{*} .
$$

Example 2.3. - Let $\mathcal{P}_{n}$ be the arrangement of $n+1$ lines in $\mathbb{C}^{2}$ defined by the polynomial $Q=x^{n+1}-y^{n+1}$. Then $\mathbb{P}\left(\mathcal{P}_{n}\right)$ consists of $n+1$ points in $\mathbb{C P}^{1}$. Thus, $U\left(\mathcal{P}_{n}\right) \cong \mathbb{C} \backslash\{n$ points $\}$, and so $M\left(\mathcal{P}_{n}\right)$ is homotopy equivalent to $S^{1} \times \bigvee^{n} S^{1}$.

2.3. Cohomology ring. - The (integral) cohomology ring of a hyperplane arrangement complement was computed by Brieskorn in [3], building on pioneering work of Arnol'd on the cohomology ring of the pure braid group. In [48], Orlik and Solomon gave a simple description of this ring, solely in terms of the intersection lattice of the arrangement.

Let $\mathcal{A}$ be an arrangement, with complement $M=M(\mathcal{A})$. Fix a linear order on $\mathcal{A}$, and let $E$ be the exterior algebra over $\mathbb{Z}$ with generators $\left\{e_{H} \mid H \in \mathcal{A}\right\}$ in degree 1. Next, define a differential $\partial: E \rightarrow E$ of degree -1 , starting from $\partial(1)=0$ and $\partial\left(e_{H}\right)=1$, and extending $\partial$ to a linear operator on $E$, using the graded Leibniz rule $\partial(a b)=\partial(a) b+(-1)^{\operatorname{deg} a} a \partial(b)$ for homogeneous elements $a$ and $b$. Finally, let $I$ be the ideal of $E$ generated by $\partial e_{\mathcal{B}}$, where $\mathcal{B}$ runs through all sub-arrangements of $\mathcal{A}$ which are not in general position, and $e_{\mathcal{B}}=\prod_{H \in \mathcal{B}} e_{H}$. Then $H^{*}(M, \mathbb{Z})$ is isomorphic, as a graded $\mathbb{Z}$-algebra, to the quotient ring $A=E / I$. 
Under this isomorphism, the basis $\left\{e_{H}\right\}$ of $A^{1}$ is dual to the basis of $H_{1}(M, \mathbb{Z})$ given by the meridians $\left\{x_{H}\right\}$ around the hyperplanes, oriented compatibly with the complex orientations on $\mathbb{C}^{d+1}$ and the hyperplanes. Furthermore, all the homology groups of $M$ are torsion-free; the generating function for their ranks is given by

$$
\operatorname{Poin}(M(\mathcal{A}), t)=\sum_{X \in L(\mathcal{A})} \mu(X)(-t)^{\operatorname{rank}(X)},
$$

where $\mu: L(\mathcal{A}) \rightarrow \mathbb{Z}$ is the Möbius function of the intersection lattice, given inductively by $\mu\left(\mathbb{C}^{d+1}\right)=1$ and $\mu(X)=-\sum_{Y \supsetneq X} \mu(Y)$. For details on all this, we refer to [49].

Recall that a finite $\mathrm{CW}$-complex is formal if its rational cohomology ring is quasiisomorphic to its Sullivan's algebra of polynomial differential forms (more details can be found in Appendix C). It follows from Brieskorn's work that the complement of a hyperplane arrangement $\mathcal{A}$ is formal, in a very strong sense. Indeed, for each hyperplane $H \in \mathcal{A}$, the 1 -form $\omega_{H}=\frac{1}{2 \pi \mathrm{i}} d \log f_{H}$ on $\mathbb{C}^{d+1}$ restricts to a 1 -form on $M(\mathcal{A})$. Let $\mathcal{D}$ be the subalgebra of the de Rham algebra $\Omega_{\mathrm{dR}}^{*}(M(\mathcal{A}))$ generated over $\mathbb{R}$ by these 1 -forms. Then, as shown in [3], the correspondence $\omega_{H} \mapsto\left[\omega_{H}\right]$ induces an isomorphism $\mathcal{D} \rightarrow H^{*}(M(\mathcal{A}), \mathbb{R})$, and this readily implies the formality of $M(\mathcal{A})$.

Similar considerations apply to the homology and cohomology of the projectivized complement, $U(\mathcal{A})$. In particular, if we let $n=|\mathcal{A}|$ be the number of hyperplanes, then $H_{1}(M(\mathcal{A}), \mathbb{Z})=\mathbb{Z}^{n}$, with canonical basis the set $\left\{x_{H}: H \in \mathcal{A}\right\}$, and

$$
H_{1}(U(\mathcal{A}), \mathbb{Z})=\mathbb{Z}^{n} /\left(\sum_{H \in \mathcal{A}} x_{H}\right) \cong \mathbb{Z}^{n-1} .
$$

We will denote by $\bar{x}_{H}$ the image of $x_{H}$ in $H_{1}(U(\mathcal{A}), \mathbb{Z})$.

2.4. Resonance varieties. - Let $\mathbb{k}$ be an algebraically closed field. The abovementioned isomorphisms allow us to identify $H^{1}(M(\mathcal{A}), \mathbb{k})$ with the affine space $\mathbb{k}^{n}$, and $H^{1}(U(\mathcal{A}), \mathbb{k})$ with the affine space

$$
\mathbb{A}_{\mathbb{k}}(\mathcal{A})=\left\{x \in \mathbb{k}^{n} \mid x_{1}+\cdots+x_{n}=0\right\} \cong \mathbb{k}^{n-1} .
$$

Let $\mathcal{R}_{s}^{q}(M(\mathcal{A}), \mathbb{k})$ be the resonance varieties of the arrangement complement. From the general theory reviewed in $\S \mathrm{A} .2$, we know that each of these sets is a homogeneous subvariety of $\mathbb{k}^{n}$, and that $\mathcal{R}_{s}^{q}(M(\mathcal{A}), \mathbb{k}) \subseteq \mathcal{R}_{1}^{q}(M(\mathcal{A}), \mathbb{k})$, for all $s \geq 1$. The diffeomorphism (3), together with the product formula (68) yields an identification

$$
\mathcal{R}_{1}^{q}(M(\mathcal{A}), \mathbb{k}) \cong \mathcal{R}_{1}^{q}(U(\mathcal{A}), \mathbb{k}) \cup \mathcal{R}_{1}^{q-1}(U(\mathcal{A}), \mathbb{k}) .
$$

Thus, we may view the resonance varieties of $\mathcal{A}$ as lying in the affine space $\mathbb{A}_{\mathrm{k}}(\mathcal{A})$.

If $\mathcal{B} \subset \mathcal{A}$ is a proper sub-arrangement, the inclusion $M(\mathcal{A}) \hookrightarrow M(\mathcal{B})$ induces an epimorphism on fundamental groups. By Proposition A.1, the induced homomorphism in cohomology restricts to an embedding $\mathcal{R}_{s}^{1}(\mathcal{B}, \mathbb{k}) \hookrightarrow \mathcal{R}_{s}^{1}(\mathcal{A}, \mathbb{k})$. The irreducible components of $\mathcal{R}_{s}^{1}(\mathcal{A}, \mathbb{k})$ that lie in the image of such an embedding are called non-essential; the remaining components are called essential.

The description of the Orlik-Solomon algebra given in $\S 2.3$ makes it clear that the resonance varieties $\mathcal{R}_{s}^{q}(M(\mathcal{A}), \mathbb{k})$ depend only on the intersection lattice, $L(\mathcal{A})$, and on the characteristic of the field $\mathbb{k}$. A basic problem in the subject is to find concrete formulas making this dependence explicit. We will briefly discuss the positive 
characteristic case in $\S 2.8$, but for now we will concentrate on the case when $\operatorname{char}(\mathbb{k})=$ 0 and $q=1$.

The complex resonance varieties $\mathcal{R}_{1}^{q}(M(\mathcal{A}), \mathbb{C})$ were first defined and studied by Falk in [23]. Soon after, Cohen-Suciu [10], Libgober [35], and Libgober-Yuzvinsky [40] showed that the varieties $\mathcal{R}_{s}(\mathcal{A})=\mathcal{R}_{s}^{1}(M(\mathcal{A}), \mathbb{C})$ consist of linear subspaces of the vector space $\mathbb{A}(\mathcal{A})=\mathbb{A}_{\mathbb{C}}(\mathcal{A})$, and analyzed the nature of these subspaces. Let us summarize those results.

Theorem 2.4. - For a hyperplane arrangement $\mathcal{A}$, the following hold.

1. Each irreducible component of $\mathcal{R}_{1}(\mathcal{A})$ is either $\{0\}$, or a linear subspace of $\mathbb{A}(\mathcal{A})$ of dimension at least 2.

2. Two distinct components of $\mathcal{R}_{1}(\mathcal{A})$ meet only at 0 .

3. $\mathcal{R}_{s}(\mathcal{A})$ is either $\{0\}$, or the union of all components of $\mathcal{R}_{1}(\mathcal{A})$ of dimension greater than $s$.

2.5. Multinets. - An elegant method, due to Falk and Yuzvinsky [25], describes explicitly the linear subspaces comprising $\mathcal{R}_{1}(\mathcal{A})$. Before proceeding, we need to introduce the relevant combinatorial notion, following the treatment from [25].

Definition 2.5. - A multinet $\mathcal{M}$ on an arrangement $\mathcal{A}$ consists of the following data:

i. An integer $k \geq 3$, and a partition of $\mathcal{A}$ into $k$ subsets, say, $\mathcal{A}_{1}, \ldots, \mathcal{A}_{k}$.

ii. An assignment of multiplicities on the hyperplanes, $m: \mathcal{A} \rightarrow \mathbb{N}$.

iii. A subset $\mathcal{X} \subseteq L_{2}(\mathcal{A})$, called the base locus.

Moreover, the following conditions must be satisfied:

1. There is an integer $\ell$ such that $\sum_{H \in \mathcal{A}_{i}} m_{H}=\ell$, for all $i \in[k]$.

2. For any two hyperplanes $H$ and $H^{\prime}$ in different classes, the flat $H \cap H^{\prime}$ belongs to $\mathcal{X}$.

3. For each $X \in \mathcal{X}$, the sum $n_{X}:=\sum_{H \in \mathcal{A}_{i}: H \supset X} m_{H}$ is independent of $i$.

4. For each $i \in[k]$, the space $\left(\bigcup_{H \in \mathcal{A}_{i}} H\right) \backslash \mathcal{X}$ is connected.

We say that a multinet as above has $k$ classes and weight $\ell$, and refer to it as a $(k, \ell)$-multinet. Without essential loss of generality, we may assume that $\operatorname{gcd}\left\{m_{H} \mid\right.$ $H \in \mathcal{A}\}=1$. If all the multiplicities are equal to 1 , the multinet is said to be reduced. If, furthermore, every flat in $\mathcal{X}$ is contained in precisely one hyperplane from each class, the multinet is called a $(k, \ell)$-net.

For any multinet on $\mathcal{A}$, the base locus $\mathcal{X}$ is determined by the partition $\left(\mathcal{A}_{1}, \ldots, \mathcal{A}_{k}\right)$. Indeed, for each $i \neq j$, we have that

$$
\mathcal{X}=\left\{H \cap H^{\prime} \mid H \in \mathcal{A}_{i}, H^{\prime} \in \mathcal{A}_{j}\right\} .
$$

The next lemma (also from [25]) is an easy consequence of this observation, and Definition 2.5. For completeness, we include a proof.

Lemma 2.6 ([25]). - For any $(k, \ell)$-multinet, the following identities hold:

1. $\sum_{H \in \mathcal{A}} m_{H}=k \ell$.

2. $\sum_{X \in \mathcal{X}: H \supset X} n_{X}=\ell$, for any $H \in \mathcal{A}$. 


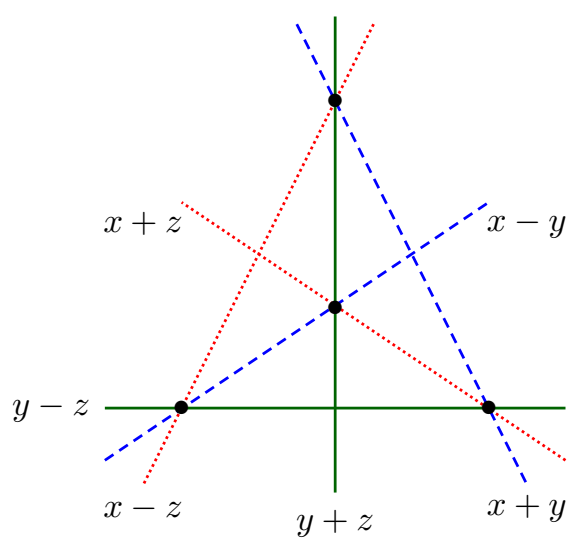

Figure 1. A $(3,2)$-net on the $\mathrm{A}_{3}$ arrangement

3. $\sum_{X \in \mathcal{X}} n_{X}^{2}=\ell^{2}$.

Proof. - Clearly,

$$
\sum_{H \in \mathcal{A}} m_{H}=\sum_{i=1}^{k} \sum_{H \in \mathcal{A}_{i}} m_{H}=k \ell
$$

To verify the second identity, let $i \in[k]$ be such that $H \in \mathcal{A}_{i}$; then, for any $j \neq i$, we have

$$
\ell=\sum_{H^{\prime} \in \mathcal{A}_{j}} m_{H^{\prime}}=\sum_{X \in \mathcal{X}: H \supset X} \sum_{H^{\prime} \in \mathcal{A}_{j}: H^{\prime} \supset X} m_{H^{\prime}}=\sum_{X \in \mathcal{X}: H \supset X} n_{X} .
$$

Finally, for any $i \neq j$,

$$
\text { (11) } \begin{aligned}
\ell^{2} & =\sum_{H \in \mathcal{A}_{i}} m_{H} \sum_{H^{\prime} \in \mathcal{A}_{j}} m_{H^{\prime}}=\sum_{H \in \mathcal{A}_{i}, H^{\prime} \in \mathcal{A}_{j}} m_{H} m_{H^{\prime}} \\
& =\sum_{H \in \mathcal{A}_{i}, H^{\prime} \in \mathcal{A}_{j}} \sum_{X \in \mathcal{X}: H \supset X, H^{\prime} \supset X} m_{H} m_{H^{\prime}}=\sum_{H \in \mathcal{A}_{i}} \sum_{X \in \mathcal{X}: H \supset X} m_{H} n_{X}=\sum_{X \in \mathcal{X}} n_{X}^{2},
\end{aligned}
$$

and this completes the proof.

Example 2.7. - Let $X \in L_{2}(\mathcal{A})$, and assume that the sub-arrangement $\mathcal{A}_{X}=$ $\{H \in \mathcal{A} \mid H \supset X\}$ has size at least 3. We may then form a net on $\mathcal{A}_{X}$ by assigning to each hyperplane the multiplicity 1 , putting one hyperplane in each class, and setting $\mathcal{X}=\{X\}$.

Example 2.8. - Let $\mathcal{A}$ be a generic 3-section of the reflection arrangement of type $\mathrm{A}_{3}$, defined by the polynomial $Q(\mathcal{A})=(x+y)(x-y)(x+z)(x-z)(y+z)(y-z)$. Figure 1 shows a plane section of $\mathcal{A}$, with the hyperplanes labeled by their defining linear forms. Ordering the hyperplanes as the factors of $Q(\mathcal{A})$, the flats in $L_{2}(\mathcal{A})$ may be labeled as $136,145,235$, and 246 . The $(3,2)$-net on $\mathcal{A}$ depicted in the picture 


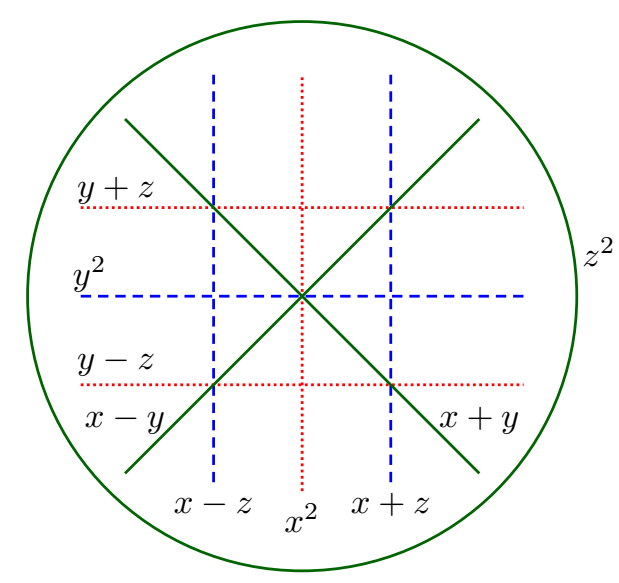

Figure 2. A $(3,4)$-multinet on the $\mathrm{B}_{3}$ arrangement

corresponds to the partition $(12|34| 56)$; the base locus $\mathcal{X}$ consists of the triple points indicated by dark circles.

Example 2.9. - Let $\mathcal{A}$ be the reflection arrangement of type $\mathrm{B}_{3}$, defined by the polynomial $Q(\mathcal{A})=x y z\left(x^{2}-y^{2}\right)\left(x^{2}-z^{2}\right)\left(y^{2}-z^{2}\right)$. Figure 2 shows a plane section of $\mathcal{A}$, together with a $(3,4)$-multinet on it.

The next theorem, which combines results of Pereira-Yuzvinsky [55] and Yuzvinsky [66], summarizes what is known about the existence of non-trivial multinets on arrangements.

Theorem 2.10. - Let $\left(\mathcal{A}_{1}, \ldots, \mathcal{A}_{k}\right)$ be a multinet on $\mathcal{A}$, with multiplicity vector $m$ and base locus $\mathcal{X}$.

1. If $|\mathcal{X}|>1$, then $k=3$ or 4 .

2. If there is a hyperplane $H \in \mathcal{A}$ such that $m_{H}>1$, then $k=3$.

Although infinite families of multinets with $k=3$ are known, only one multinet with $k=4$ is known to exist: the $(4,3)$-net on the Hessian arrangement, which we will discuss in Example 2.15. In fact, it is a conjecture of Yuzvinsky [67] that the only $(4, \ell)$-multinet is the Hessian $(4,3)$-net.

2.6. Resonance and multinets. - We now return to the (degree 1$)$ complex resonance varieties of a hyperplane arrangement $\mathcal{A}$. Recall that $\mathcal{R}_{1}(\mathcal{A})=\mathcal{R}_{1}^{1}(M(\mathcal{A}), \mathbb{C})$ is a subvariety of the affine space $\mathbb{A}(\mathcal{A})=\left\{x \in \mathbb{C}^{|\mathcal{A}|} \mid \sum_{H \in \mathcal{A}} x_{H}=0\right\}$, consisting of linear subspaces meeting only at the origin. Let us describe these subspaces in a concrete way.

Given a multinet $\mathcal{M}$ on $\mathcal{A}$, with parts $\left(\mathcal{A}_{1}, \ldots, \mathcal{A}_{k}\right)$ and multiplicity vector $m$, set $u_{i}=\sum_{H \in \mathcal{A}_{i}} m_{H} e_{H}$ for each $1 \leq i \leq k$, and put

$$
P_{\mathcal{M}}=\operatorname{span}\left\{u_{2}-u_{1}, \ldots, u_{k}-u_{1}\right\} .
$$


By construction, $P_{\mathcal{M}}$ is a linear subspace of $\mathbb{A}(\mathcal{A})$. As shown in [25, Theorem 2.4], this subspace lies inside $\mathcal{R}_{1}(\mathcal{A})$ and has dimension $k-1$.

Now suppose there is a sub-arrangement $\mathcal{B} \subset \mathcal{A}$ which supports a multinet $\mathcal{M}$ with $k$ parts. By the above, the linear space $P_{\mathcal{M}}$ lies inside $\mathcal{R}_{1}(\mathcal{B})$. By the discussion from $\S 2.4$, the inclusion $M(\mathcal{A}) \hookrightarrow M(\mathcal{B})$ induces an embedding $\mathcal{R}_{1}(\mathcal{B}) \hookrightarrow \mathcal{R}_{1}(\mathcal{A})$. Thus, $P_{\mathcal{M}}$ is a linear subspace of $\mathcal{R}_{1}(\mathcal{A})$, of dimension $k-1$. Conversely, it is shown in [25, Theorem 2.5] that all (non-zero) irreducible components of $\mathcal{R}_{1}(\mathcal{A})$ arise in this fashion.

Summarizing the above discussion, we have the following description of the first resonance variety of an arrangement.

Theorem 2.11 ([25]). - The positive-dimensional, irreducible components of $\mathcal{R}_{1}(\mathcal{A})$ are in one-to-one correspondence with the multinets on sub-arrangements of $\mathcal{A}$, and so

$$
\mathcal{R}_{1}(\mathcal{A})=\{0\} \cup \bigcup_{\mathcal{B} \subset \mathcal{A} \mathcal{M} \text { a multinet on } \mathcal{B}} P_{\mathcal{M}}
$$

Using now Theorem 2.4, part 3, we find that

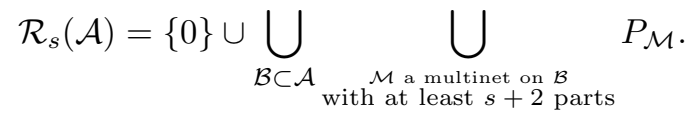

2.7. Local and non-local components. - The simplest components of $\mathcal{R}_{1}(\mathcal{A})$ arise in the following fashion. Let $X$ be a rank 2 flat lying at the intersection of at least 3 hyperplanes. Recall from Example 2.7 that $X$ determines a net on the sub-arrangement $\mathcal{A}_{X}$ consisting of those hyperplanes in $\mathcal{A}$ that contain $X$. The corresponding component of $\mathcal{R}_{1}(\mathcal{A})$,

$$
P_{X}=\left\{x \in \mathbb{A}(\mathcal{A}) \mid \sum_{H \supset X} x_{H}=0 \text { and } x_{H}=0 \text { if } H \not \supset X\right\},
$$

has dimension $\left|\mathcal{A}_{X}\right|-1$, and is called a local component.

If $|\mathcal{A}| \leq 5$, then all components of $\mathcal{R}_{1}(\mathcal{A})$ are local. For $|\mathcal{A}| \geq 6$, though, the resonance variety $\mathcal{R}_{1}(\mathcal{A})$ may have non-local components. It follows from Theorems 2.10 and 2.11 that any such component must have dimension either 2 or 3 .

Example 2.12. - Let $\mathcal{A}$ be the braid arrangement from Example 2.8. The variety $\mathcal{R}_{1}(\mathcal{A}) \subset \mathbb{C}^{6}$ has 4 local components, corresponding to the flats $136,145,235,246$, and one non-local component, corresponding to the net $\mathcal{M}$ indicated in Figure 1:

$$
\begin{aligned}
& P_{136}=\left\{x_{1}+x_{3}+x_{6}=x_{2}=x_{4}=x_{5}=0\right\}, P_{145}=\left\{x_{1}+x_{4}+x_{5}=x_{2}=x_{3}=x_{6}=0\right\}, \\
& P_{235}=\left\{x_{2}+x_{3}+x_{5}=x_{1}=x_{4}=x_{6}=0\right\}, P_{246}=\left\{x_{2}+x_{4}+x_{6}=x_{1}=x_{3}=x_{5}=0\right\}, \\
& P_{\mathcal{M}}=\left\{x_{1}+x_{3}+x_{6}=x_{1}-x_{2}=x_{3}-x_{4}=x_{5}-x_{6}=0\right\} .
\end{aligned}
$$

Since all these components are 2-dimensional, $\mathcal{R}_{2}(\mathcal{A})=\{0\}$.

Example 2.13. - Let $\mathcal{A}$ be the $\mathrm{B}_{3}$-arrangement from Example 2.9. Ordering the hyperplanes as the factors of the defining polynomial, the multinet $\mathcal{M}$ indicated in Figure 2 has associated partition (167|289|345). 


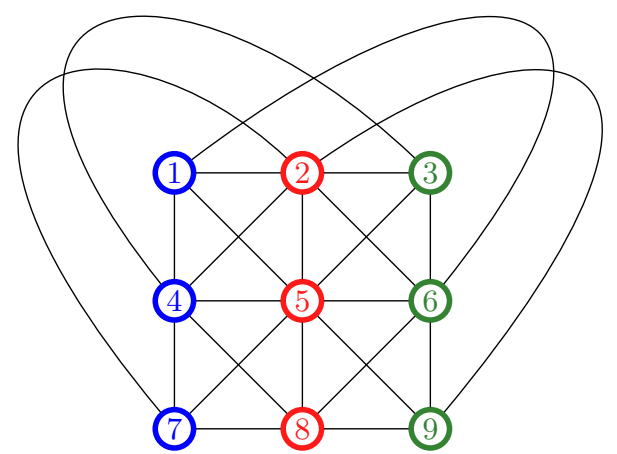

Figure 3. The Ceva(3) matroid, together with a $(3,3)$-net

The variety $\mathcal{R}_{1}(\mathcal{A}) \subset \mathbb{C}^{9}$ has 7 local components, corresponding to 4 triple points and 3 quadruple points, 11 components corresponding to braid sub-arrangements, and one essential, 2-dimensional component, corresponding to the above multinet,

$$
P_{\mathcal{M}}=\left\{x_{1}=x_{6}=x_{7}, x_{2}=x_{8}=x_{9}, x_{3}=x_{4}=x_{5}, x_{1}+x_{2}+x_{3}=0\right\} .
$$

Example 2.14. - Let $\mathcal{A}$ be the Ceva(3) arrangement, also known as the monomial arrangement of type $\mathcal{A}(3,3,3)$, associated to the complex reflection group $G(3,3,3)$, and defined by the polynomial $Q(\mathcal{A})=\left(x^{3}-y^{3}\right)\left(y^{3}-z^{3}\right)\left(x^{3}-z^{3}\right)$. Figure 3 shows the corresponding matroid, denoted $\mathrm{AG}(2,3)$ in [50].

The resonance variety $\mathcal{R}_{1}(\mathcal{A}) \subset \mathbb{C}^{9}$ has 12 local components, corresponding to the triple points, and 4 essential components corresponding to the $(3,3)$-nets defined by the partitions $(123|456| 789)$, (147|258|369), (159|267|348), and (168|249|357). The first of these nets is depicted in Figure 3.

The next example describes the only arrangement for which non-local components of dimension 3 are known to occur.

Example 2.15. - Let $\mathcal{A}$ be the Hessian arrangement in $\mathbb{C}^{3}$, also known as the monomial arrangement of type $\mathcal{A}(3,1,3)$ associated to the complex reflexion group of type $G(3,1,3)$, and defined by the polynomial $Q(\mathcal{A})=x y x\left(x^{3}-y^{3}\right)\left(x^{3}-z^{3}\right)\left(y^{3}-z^{3}\right)$. The projective configuration consists of 12 projective lines meeting at 9 quadruple points, and 12 double points; each line contains 3 quadruple points and 2 double points. Figure 4 shows the corresponding matroid, which is obtained from the projective plane over $\mathbb{Z}_{3}$ (also known as the $\mathrm{PG}(2,3)$ matroid, cf. [50]) by deleting a point. The resonance variety $\mathcal{R}_{1}(\mathcal{A})$ has 9 local components, and a single, 3-dimensional, essential component, corresponding to the $(4,3)$-net depicted in Figure 4.

2.8. Resonance in positive characteristic. - The varieties $\mathcal{R}_{s}^{1}(M(\mathcal{A}), \mathbb{k})$ over fields $\mathbb{k}$ of characteristic $p>0$ were first defined and studied by Matei and Suciu in [43], and further investigated by Falk in [24]. As illustrated in the next few examples, all three properties from Theorem 2.4 , which hold over $\mathbb{k}=\mathbb{C}$, fail in this setting. 


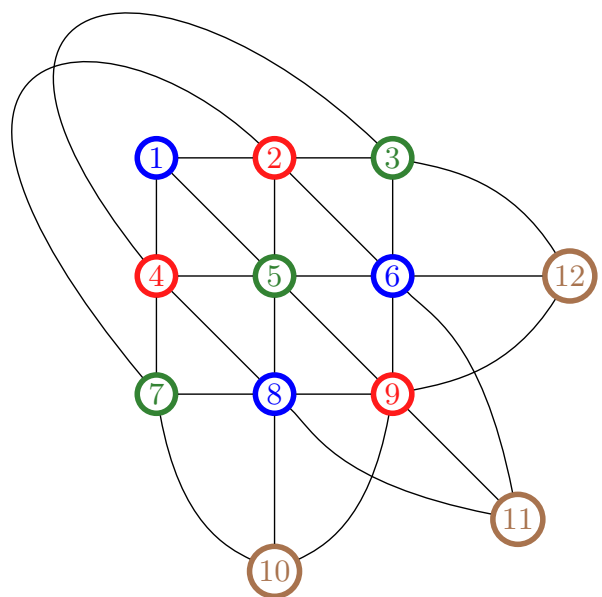

Figure 4. The Hessian matroid, together with a (4,3)-net

Example 2.16. - Let $\mathcal{A}$ be the realization of the non-Fano plane, obtained from the $\mathrm{B}_{3}$ arrangement from Figure 2 by deleting the planes $x=0$ and $y=0$, and order the hyperplanes as the factors of $Q(\mathcal{A})=z(x+y)(x-y)(x+z)(x-z)(y+z)(y-z)$. The variety $\mathcal{R}_{1}(\mathcal{A})$ has 6 local components corresponding to triple points, and 3 components arising from braid sub-arrangements, but no essential components.

Now let $\mathbb{k}=\overline{\mathbb{F}_{2}}$. Then, as noted in [43], the variety $\mathcal{R}_{1}^{1}(M(\mathcal{A}), \mathbb{k}) \subset \mathbb{A}_{\mathbb{k}}(\mathcal{A})$ has a single non-local component, defined by the equations $x_{1}+x_{4}+x_{5}=x_{1}+x_{6}+x_{7}=$ $x_{2}+x_{5}+x_{6}=x_{3}+x_{5}+x_{7}=0$, while $\mathcal{R}_{2}^{1}(M(\mathcal{A}), \mathbb{k})$ has a single, 1-dimensional component, defined by the equations $x_{4}=x_{5}=x_{6}=x_{7}$ and $x_{1}=x_{2}=x_{3}=0$. Thus, property 3 fails.

It should be noted that the analogue of the tangent cone formula (81) also fails in this case. Indeed, a computation from [58] shows that $\mathcal{V}_{2}^{1}(M(\mathcal{A}), \mathbb{k})=\{1\}$; thus, the tangent cone to this variety is $\{0\}$, which is is strictly included in $\mathcal{R}_{2}^{1}(M(\mathcal{A}), \mathbb{k})$.

Example 2.17. - Let $\mathcal{A}$ be the arrangement obtained by deleting the plane $z=0$ from the $\mathrm{B}_{3}$ arrangement, and let $\mathbb{k}=\overline{\mathbb{F}_{2}}$. Then, as shown in [24], the variety $\mathcal{R}_{1}^{1}(M(\mathcal{A}), \mathbb{k})$ has two distinct components which intersect outside 0 . Thus, property 2 fails.

Example 2.18. - Let $\mathcal{A}$ be the Hessian arrangement from Example 2.15, and let $\mathbb{k}=\overline{\mathbb{F}_{3}}$. Then, as shown in [24], the variety $\mathcal{R}_{1}^{1}(M(\mathcal{A}), \mathbb{k})$ has a component which is isomorphic to an irreducible cubic hypersurface in $\mathbb{k}^{5}$. Thus, property 1 fails.

Remark 2.19. - In [42], Matei uses the resonance varieties $\mathcal{R}_{1}^{1}(M(\mathcal{A}), \mathbb{k})$ over a field $\mathbb{k}$ of characteristic an odd prime $p$ to detect non-vanishing Massey products in the cohomology ring $H^{*}(M(\mathcal{A}), \mathbb{k})$ of certain arrangements $\mathcal{A}$. The simplest such example is the Hessian arrangement from Example 2.15, for which $p=3$.

These examples and the above remark raise a natural question. 
Problem 2.20. - Given an arrangement $\mathcal{A}$, find the primes $p$ (if any) for which $M(\mathcal{A})$ is not $\mathbb{k}$-formal, over a field $\mathbb{k}$ of characteristic $p$.

\section{The complement of an arrangement. II.}

In this section we describe the fundamental group and the characteristic varieties of the complement of a complex hyperplane arrangement.

3.1. Fundamental group. - As usual, let $\mathcal{A}$ be a hyperplane arrangement in $\mathbb{C}^{d+1}$. The complement $M(\mathcal{A})$ is a path-connected space. Thus, we may fix a basepoint $x_{0} \in M(\mathcal{A})$, and consider the fundamental group $\pi_{1}\left(M(\mathcal{A}), x_{0}\right)$.

For each hyperplane $H \in \mathcal{A}$, pick a meridian curve about $H$, oriented compatibly with the complex orientations on $\mathbb{C}^{d+1}$ and $H$, and let $x_{H}$ denote the based homotopy class of this curve, joined to the basepoint by a path in $M(\mathcal{A})$. By the van Kampen theorem, then, the group $\pi_{1}\left(M(\mathcal{A}), x_{0}\right)$ is generated by the set $\left\{x_{H}: H \in \mathcal{A}\right\}$. An explicit presentation for this group can be obtained via the braid monodromy algorithm from [9]. Let us briefly describe this algorithm.

Let $\mathcal{A}^{\prime}$ be a generic two-dimensional section of $\mathcal{A}$. By the Lefschetz-type theorem of Hamm and Lê, the inclusion $M\left(\mathcal{A}^{\prime}\right) \rightarrow M(\mathcal{A})$ between the respective complements induces an isomorphism on fundamental groups. Thus, for the purpose of computing fundamental groups, we may as well replace $\mathcal{A}$ by $\mathcal{A}^{\prime}$.

So let $\mathcal{A}=\left\{\ell_{1}, \ldots, \ell_{n}\right\}$ be an arrangement of affine lines in $\mathbb{C}^{2}$. Let $v_{1}, \ldots, v_{s}$ be the intersection points of the lines. If $v=\ell_{i_{1}} \cap \cdots \cap \ell_{i_{r}}$ is one such intersection point, write $I=\left(i_{1}, \ldots, i_{r}\right)$ for the corresponding increasingly ordered tuple, and let $A_{I}$ be the braid in the pure braid group $P_{n}$ which performs a full twist on the strands corresponding to $I$, leaving the other strands fixed. There are then braids $\delta_{1}, \ldots, \delta_{s}$ in the full braid group $B_{n}$ such that the arrangement group has presentation

(15) $\pi_{1}(M(\mathcal{A}))=\left\langle x_{1}, \ldots, x_{n}\right| \delta_{q}^{-1} A_{I_{s}} \delta_{q}\left(x_{i}\right)=x_{i}$ for $i \in I_{q} \backslash \max \left(I_{q}\right)$ and $\left.q \in[s]\right\rangle$.

Clearly, this is a commutator-relators presentation. Furthermore, the presentation is minimal, in that the number of generators equals $b_{1}(M(\mathcal{A}))$, and the number of relators equals $b_{2}(M(\mathcal{A}))$.

If $\mathcal{A}$ is the complexification of a real arrangement, the conjugating braids $\delta_{q}$ may be obtained by a procedure that goes back to [28]. Each vertex set $I_{q}$ gives rise to a partition $[n]=I_{q}^{\prime} \cup I_{q} \cup I_{q}^{\prime \prime}$ into lower, middle, and upper indices. Let $J_{q}=\left\{i \in I_{q}^{\prime \prime} \mid\right.$ $\left.\min I_{q}<i<\max I_{q}\right\}$. Then $\delta_{q}$ is the subword of the full twist $A_{[n]}$ given by

$$
\delta_{q}=\prod_{i \in I_{q}} \prod_{j \in J_{q}} A_{j i} .
$$

In the general case, the braids $\delta_{q}$ can be read off a "braided wiring diagram" associated to the arrangement, see [9] for further details and references.

Remark 3.1. - In work from the early 1990s that appeared in [56], Rybnikov constructed a pair of arrangements, $\mathcal{A}^{+}$and $\mathcal{A}^{-}$, both realizations of the same matroid, for which $\pi_{1}\left(M\left(\mathcal{A}^{+}\right)\right) \neq \pi_{1}\left(M\left(\mathcal{A}^{-}\right)\right)$. Thus, the fundamental group of an arrangement complement is not determined by the intersection lattice, in general. 
Remark 3.2. - Nevertheless, if $G=\pi_{1}(M(\mathcal{A}))$ is an arrangement group, the ranks of the associated graded Lie algebra, $\phi_{k}=\operatorname{rank}\left(\operatorname{gr}_{k}(G)\right)$, are combinatorially determined, due to the formality of $M(\mathcal{A})$. Moreover, as shown in [51], the ranks of the Chen Lie algebra, $\theta_{k}=\operatorname{rank}\left(\operatorname{gr}_{k}\left(G / G^{\prime \prime}\right)\right)$, are also combinatorially determined. As observed in [58], there are interesting connections between the Chen ranks of the group $G$ and the dimensions of the components of the resonance variety $\mathcal{R}_{1}(\mathcal{A})$. For a recent survey of these topics, we refer to [13].

3.2. The universal abelian cover. - Returning to the general situation, let $\mathcal{A}$ be an essential arrangement in $\mathbb{C}^{d+1}$. Fix an ordering of the hyperplanes, say, $\mathcal{A}=$ $\left\{H_{1}, \ldots, H_{n}\right\}$, and choose linear forms $f_{i}: \mathbb{C}^{d+1} \rightarrow \mathbb{C}$ with $\operatorname{ker}\left(f_{i}\right)=H_{i}$. Assembling these forms together, we obtain an injective linear map

$$
\iota(\mathcal{A}): \mathbb{C}^{d+1} \rightarrow \mathbb{C}^{n}, \quad z \mapsto\left(f_{1}(z), \ldots, f_{n}(z)\right) .
$$

Now let $\mathcal{B}_{n}$ be the Boolean arrangement in $\mathbb{C}^{n}$, and identify $M\left(\mathcal{B}_{n}\right)$ with $\left(\mathbb{C}^{*}\right)^{n}$. Recall that $z \in H_{i}$ if and only if $f_{i}(z)=0$; thus, the map $\iota(\mathcal{A})$ restricts to an inclusion $\iota(\mathcal{A}): M(\mathcal{A}) \hookrightarrow M\left(\mathcal{B}_{n}\right)$. Consequently, we may view the complement $M(\mathcal{A})$ as a linear slice of the complex $n$-torus:

$$
M(\mathcal{A})=\iota(\mathcal{A})\left(\mathbb{C}^{d+1}\right) \cap\left(\mathbb{C}^{*}\right)^{n} .
$$

Clearly, the map $\iota(\mathcal{A}): M(\mathcal{A}) \rightarrow\left(\mathbb{C}^{*}\right)^{n}$ is equivariant with respect to the diagonal action of $\mathbb{C}^{*}$ on both source and target. Thus, $\iota(\mathcal{A})$ descends to a map $\bar{\iota}(\mathcal{A}): M(\mathcal{A}) / \mathbb{C}^{*} \hookrightarrow\left(\mathbb{C}^{*}\right)^{n} / \mathbb{C}^{*}$, which defines an embedding $\bar{\iota}(\mathcal{A}): U(\mathcal{A}) \hookrightarrow\left(\mathbb{C}^{*}\right)^{n-1}$.

Lemma $3.3([14])$ - Let $\mathcal{A}=\left\{H_{1}, \ldots, H_{n}\right\}$ be a hyperplane arrangement. Then,

1. The inclusion $\iota(\mathcal{A}): M(\mathcal{A}) \hookrightarrow\left(\mathbb{C}^{*}\right)^{n}$ is a classifying map for the universal abelian cover $M(\mathcal{A})^{\mathrm{ab}} \rightarrow M(\mathcal{A})$.

2. The inclusion $\bar{\iota}(\mathcal{A}): U(\mathcal{A}) \hookrightarrow\left(\mathbb{C}^{*}\right)^{n-1}$ is a classifying map for the universal abelian cover $U(\mathcal{A})^{\mathrm{ab}} \rightarrow U(\mathcal{A})$.

Proof. - We start with the first statement. Set $\iota=\iota(\mathcal{A})$. Since $\left(\mathbb{C}^{*}\right)^{n}$ is a classifying space for the group $\pi_{1}(M(\mathcal{A}))_{\mathrm{ab}}=\mathbb{Z}^{n}$, it is enough to to check that the induced homomorphism, $\iota_{\sharp}: \pi_{1}(M(\mathcal{A})) \rightarrow \mathbb{Z}^{n}$, coincides with the abelianization map ab: $\pi_{1}(M(\mathcal{A})) \rightarrow \pi_{1}(M(\mathcal{A}))_{\mathrm{ab}}$. By naturality of the Hurewicz homomorphism, we have a commuting diagram

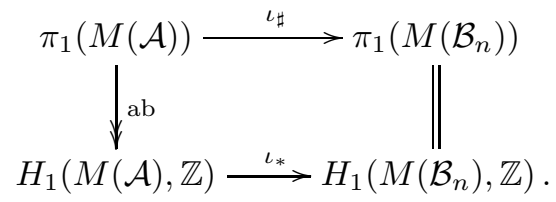

Plainly, the homomorphism $\iota_{*}$ takes each meridian $x_{H}$ in $H_{1}(M(\mathcal{A}), \mathbb{Z})=\mathbb{Z}^{n}$ to the corresponding meridian in $H_{1}\left(M\left(\mathcal{B}_{n}\right), \mathbb{Z}\right)=\mathbb{Z}^{n}$. Hence, in these meridian bases, the map $\iota_{*}: \mathbb{Z}^{n} \rightarrow \mathbb{Z}^{n}$ is the identity map, and we are done.

For the second statement, recall from $(5)$ that $H_{1}(U(\mathcal{A}), \mathbb{Z})=\mathbb{Z}^{n} /\left(\sum_{H \in \mathcal{A}} x_{H}\right)$. An argument as above shows that $\bar{\iota}_{\sharp}(\mathcal{A}): \pi_{1}(U(\mathcal{A})) \rightarrow \mathbb{Z}^{n} /(1, \ldots, 1)$ is the abelianization map, and this ends the proof. 
3.3. Characteristic varieties. - Let $G=\pi_{1}(M(\mathcal{A}))$ and $\bar{G}=\pi_{1}(U(\mathcal{A}))$ be the fundamental groups of the complement and the projectivized complement, respectively. Let $\mathbb{k}$ be an algebraically closed field. The isomorphism $H_{1}(M(\mathcal{A}), \mathbb{Z}) \cong \mathbb{Z}^{n}$ allows us to identify the character group $\operatorname{Hom}\left(G, \mathbb{k}^{*}\right)$ with the algebraic torus $\left(\mathbb{k}^{*}\right)^{n}$. Similarly, the isomorphism $H_{1}(U(\mathcal{A}), \mathbb{Z}) \cong \mathbb{Z}^{n} /(1, \ldots, 1)$ allows us to identify the character group $\operatorname{Hom}\left(\bar{G}, \mathbb{k}^{*}\right)$ with the algebraic torus

$$
\mathbb{T}_{\mathbb{k}}(\mathcal{A})=\left\{t \in\left(\mathbb{k}^{*}\right)^{n} \mid t_{1} \cdots t_{n}=1\right\} \cong\left(\mathbb{k}^{*}\right)^{n-1} .
$$

Let $\mathcal{V}_{s}^{q}(M(\mathcal{A}), \mathbb{k})$ be the characteristic varieties of the arrangement complement. From the general theory reviewed in $\S$ A.1, we know that each set $\mathcal{V}_{s}^{q}(M(\mathcal{A}), \mathbb{k})$ is a Zariski closed subset of the algebraic torus $\left(\mathbb{k}^{*}\right)^{n}$. The diffeomorphism $(3)$, together with the product formula (65) yields an identification $\mathcal{V}_{1}^{q}(M(\mathcal{A}), \mathbb{k}) \cong \mathcal{V}_{1}^{q}(U(\mathcal{A}), \mathbb{k}) \cup$ $\mathcal{V}_{1}^{q-1}(U(\mathcal{A}), \mathbb{k})$. In particular, we may view the characteristic varieties of $\mathcal{A}$ as lying in the torus (20).

Example 3.4. - Let $\mathcal{A}$ be the arrangement in $\mathbb{C}$ consisting of the single point 0. Then $M(\mathcal{A})=\mathbb{C}^{*}$ is homotopy equivalent to a circle $S^{1}$. Under the identifications $\pi_{1}\left(S^{1}, 1\right)=\mathbb{Z}$ and $\mathbb{Z} \mathbb{Z}=\mathbb{Z}\left[t^{ \pm 1}\right]$, the equivariant chain complex of the universal cover $\widetilde{S^{1}}=\mathbb{R}$ takes the form $0 \rightarrow C_{1} \stackrel{\partial_{1}}{\longrightarrow} C_{0} \rightarrow 0$, where $C_{1}=C_{0}=\mathbb{Z} \mathbb{Z}$ and $\partial_{1}(1)=t-1$.

Given a character $\rho \in \operatorname{Hom}\left(\mathbb{Z}, \mathbb{k}^{*}\right)=\mathbb{k}^{*}$, we tensor this chain complex with the local system $\mathbb{k}_{\rho}$, and obtain a new chain complex, $0 \rightarrow \mathbb{k} \stackrel{\rho-1}{\longrightarrow} \mathbb{k} \rightarrow 0$. This chain complex is exact, except for $\rho=1$, when $H_{0}=H_{1}=\mathbb{k}$. The upshot is that $\mathcal{V}_{1}^{0}\left(S^{1}, \mathbb{k}\right)=$ $\mathcal{V}_{1}^{1}\left(S^{1}, \mathbb{k}\right)=\{1\}$ and $\mathcal{V}_{s}^{i}\left(S^{1}, \mathbb{k}\right)=\emptyset$, otherwise.

Example 3.5. - More generally, let $\mathcal{B}_{n}$ be the Boolean arrangement in $\mathbb{C}^{n}$, with complement $M\left(\mathcal{B}_{n}\right)=\left(\mathbb{C}^{*}\right)^{n}$. Proceeding as in the previous example, we see that

$$
\mathcal{V}_{s}^{q}\left(M\left(\mathcal{B}_{n}\right), \mathbb{k}\right)= \begin{cases}\{1\} & \text { if } s \leq\left(\begin{array}{l}
n \\
q
\end{array}\right) \\
\emptyset & \text { otherwise }\end{cases}
$$

Example 3.6. - Let $\mathcal{P}_{n}$ be a pencil of $n+1 \geq 2$ lines in $\mathbb{C}^{2}$ as in Example 2.3. Recall that $U\left(\mathcal{P}_{n}\right) \cong \mathbb{C} \backslash\{n$ points $\}$, and thus $\pi_{1}\left(U\left(\mathcal{P}_{n}\right)\right) \cong F_{n}$. Identifying $\mathbb{T}_{\mathbb{k}}\left(\mathcal{P}_{n}\right)$ with $\left(\mathbb{k}^{*}\right)^{n}$, we find that

$$
\mathcal{V}_{s}^{q}\left(M\left(\mathcal{P}_{n}\right), \mathbb{k}\right)= \begin{cases}\left(\mathbb{k}^{*}\right)^{n} & \text { if } q=1 \text { and } s<n \\ \{1\} & \text { if } q=1 \text { and } s=n \text { or } q=0 \text { and } s=1 \\ \emptyset & \text { otherwise. }\end{cases}
$$

Although an explicit formula for the characteristic varieties $\mathcal{V}_{s}^{q}(M(\mathcal{A}), \mathbb{k})$ is far from known in general, a structural result is known in the case when $\mathbb{k}=\mathbb{C}$. A theorem of Arapura [1], as strengthened by Dimca [17], Libgober [38], Artal-Bartolo, Cogolludo and Matei [2], Budur and Wang [5], and others, states the following.

Theorem 3.7. - The characteristic varieties $\mathcal{V}_{s}^{q}(M(\mathcal{A}), \mathbb{C})$ of an arrangement complement are finite unions of torsion-translates of algebraic subtori in $\mathbb{T}_{\mathbb{C}}(\mathcal{A})$. 
3.4. Orbifold fibrations. - We now describe in more detail the characteristic varieties of $M(\mathcal{A})$ in degree $q=1$. Since these varieties depend only on $\pi_{1}(M(\mathcal{A})$ ), we may as well assume $\mathcal{A}$ is a (central) arrangement in $\mathbb{C}^{3}$.

A key point here is the naturality property enjoyed by these varieties. Suppose $f: U(\mathcal{A}) \rightarrow U(\mathcal{B})$ is a map between (projectivized) arrangement complements, and that the induced homomorphism, $f_{\sharp}: \pi_{1}(U(\mathcal{A})) \rightarrow \pi_{1}(U(\mathcal{B}))$, is surjective. Then, by Proposition A.1, the corresponding monomorphism between character groups restricts to an embedding $\mathcal{V}_{s}^{1}(\mathcal{B}, \mathbb{k}) \hookrightarrow \mathcal{V}_{s}^{1}(\mathcal{A}, \mathbb{k})$. In particular, if $\mathcal{B} \subset \mathcal{A}$ is a sub-arrangement, the inclusion $U(\mathcal{A}) \hookrightarrow U(\mathcal{B})$ induces an epimorphism on fundamental groups, and thus defines an embedding $\mathcal{V}_{s}^{1}(\mathcal{B}, \mathbb{k}) \hookrightarrow \mathcal{V}_{s}^{1}(\mathcal{A}, \mathbb{k})$.

Building on Arapura's work, Dimca [17] and Artal Bartolo, Cogolludo and Matei [2] showed that the varieties $\mathcal{V}_{s}(\mathcal{A})=\mathcal{V}_{s}^{1}(M(\mathcal{A}), \mathbb{C})$ are unions of torsion-translated subtori inside the algebraic torus $\mathbb{T}(\mathcal{A})=\mathbb{T}_{\mathbb{C}}(\mathcal{A})$. The isolated (torsion) points in these characteristic varieties are still poorly understood, but the positive-dimensional components in $\mathcal{V}_{1}(\mathcal{A})$ can be described in very concrete terms.

A (genus 0 ) orbifold fibration is a surjective holomorphic map $f: U(\mathcal{A}) \rightarrow U\left(\mathcal{P}_{r}\right)$, for some $r \geq 1$, that has connected generic fiber, and extends to a map between the respective compactifications, $\bar{f}: \mathbb{C P}^{2} \rightarrow \mathbb{C P}^{1}$, which is also a surjective, holomorphic map with connected generic fibers.

The map $f$ is a locally trivial bundle map, away from a finite (possibly empty) set of points $\left\{q_{1}, \ldots, q_{s}\right\}$ inside $U\left(\mathcal{P}_{r}\right)$; let $\mu_{1}, \ldots, \mu_{s}\left(\mu_{i} \geq 2\right)$ denote the multiplicities of the respective fibers. Let $f_{\sharp}: \pi_{1}(U(\mathcal{A})) \rightarrow \pi_{1}\left(U\left(\mathcal{P}_{r}\right)\right)$ be the induced homomorphism on fundamental groups. Since the generic fiber of $f$ is connected, this homomorphism is surjective. Moreover, $f_{\sharp}$ lifts to a (surjective) homomorphism $f_{\sharp}: \pi_{1}(U(\mathcal{A})) \rightarrow \pi$, where

$$
\pi:=\pi_{1}^{\text {orb }}\left(U\left(\mathcal{P}_{r}\right), \mu\right)=F_{r} * \mathbb{Z}_{\mu_{1}} * \cdots * \mathbb{Z}_{\mu_{s}}
$$

is the orbifold fundamental group of the base. Thus, $f_{\sharp}$ induces an injective morphism, $\left.\widehat{f}_{\sharp}: \widehat{\pi} \hookrightarrow \pi_{1} \widehat{(U(\mathcal{A})}\right)$, between character groups. Note that $\widehat{\pi}=\widehat{\pi}^{\circ} \times \widehat{A}$ where $\widehat{\pi}^{\circ} \cong\left(\mathbb{C}^{*}\right)^{r}$ is the identity component and $\widehat{A} \cong A:=\mathbb{Z}_{\mu_{1}} \oplus \cdots \oplus \mathbb{Z}_{\mu_{s}}$.

By Proposition A.1, the above morphism restricts to an inclusion $\widehat{f}_{\sharp}: \mathcal{V}_{1}(\pi) \hookrightarrow$ $\mathcal{V}_{1}(\mathcal{A})$. A computation as in Example 3.4 shows that

$$
\mathcal{V}_{1}(\pi)= \begin{cases}\widehat{\pi} & \text { if } r>1, \\ \left(\widehat{\pi} \backslash \widehat{\pi}^{\circ}\right) \cup\{\mathbf{1}\} & \text { if } r=1 \text { and } s \geq 1 .\end{cases}
$$

Note that each irreducible component of $\mathcal{V}_{1}(\pi)$ has dimension $r$.

The next theorem says that all positive-dimensional components of $\mathcal{V}_{1}(\mathcal{A})$ arise by pullback along such orbifold fibrations, which we call large in the first case, and small in the second case.

Theorem $3.8([1,17,2])$. - The first characteristic variety of an arrangement $\mathcal{A}$ is given by

$$
\mathcal{V}_{1}(\mathcal{A})=\bigcup_{f \text { large }} \operatorname{im}\left(\widehat{f}_{\sharp}\right) \cup \bigcup_{f \text { small }}\left(\operatorname{im}\left(\widehat{f}_{\sharp}\right) \backslash \operatorname{im}\left(\widehat{f}_{\sharp}\right)^{\circ}\right) \cup Z,
$$


where the unions are over the equivalence classes of pencils $f: U(\mathcal{A}) \rightarrow U\left(\mathcal{P}_{r}\right)$ of the types indicated, and $Z$ is a finite set of torsion characters.

3.5. Multinets and pencils. - As shown by Falk and Yuzvinsky in [25], the irreducible components of $\mathcal{V}_{1}(\mathcal{A})$ passing through the origin can be described in terms of multinets on the intersection lattice of $\mathcal{A}$. Indeed, let $Q(\mathcal{A})=\prod_{H \in \mathcal{A}} f_{H}$ be a defining polynomial for $\mathcal{A}$. Given a multinet $\mathcal{M}$ on $\mathcal{A}$, with parts $\left(\mathcal{A}_{1}, \ldots, \mathcal{A}_{k}\right)$ and multiplicity vector $m$, write

$$
g_{i}=\prod_{H \in \mathcal{A}_{i}} f_{H}^{m_{H}},
$$

for $1 \leq i \leq k$, so that the defining polynomial for $(\mathcal{A}, m)$ factors as $Q_{m}(\mathcal{A})=g_{1} \cdots g_{k}$. Next, define a rational map $f_{m}: \mathbb{C}^{3} \rightarrow \mathbb{P}^{1}$ by

$$
f_{m}(x)=\left[g_{1}(x): g_{2}(x)\right] .
$$

By definition of multinets, the degrees of the polynomials $g_{i}$ are independent of $i$; hence, the map $f_{m}$ factors through a rational map $\mathbb{C P}^{2} \rightarrow \mathbb{C P}^{1}$. As shown in [25], there is a set $S=\left\{\left[a_{1}: b_{1}\right], \ldots,\left[a_{k}: b_{k}\right]\right\} \subset \mathbb{C P}^{1}$ such that each of the polynomials $(25)$ can be written as $g_{i}=a_{i} g_{2}-b_{i} g_{1}$, and, furthermore, the image of $f_{m}: U(\mathcal{A}) \rightarrow \mathbb{C P}^{1}$ misses $S$. Identify $\mathbb{C P}^{1} \backslash S=U\left(\mathcal{P}_{k-1}\right)$. The restriction

$$
f_{m}: U(\mathcal{A}) \rightarrow U\left(\mathcal{P}_{k-1}\right),
$$

then, is a large pencil which gives rise by pullback to a $(k-1)$-dimensional component of $\mathcal{V}_{1}(\mathcal{A})$ which passes through the origin of $\mathbb{T}(\mathcal{A})$.

Example 3.9. - Let $\mathcal{A}$ be the braid arrangement from Examples 2.8 and 2.12. The characteristic variety $\mathcal{V}_{1}(\mathcal{A})$ has 4 local components of dimension 2 , corresponding to the 4 triple points. Additionally, the $(3,2)$-net $(\mathcal{A}, m)$ depicted in Figure 1 defines a rational map, $f_{m}: \mathbb{C P}^{2} \rightarrow \mathbb{C P}^{1},(x, y, z) \mapsto\left(x^{2}-y^{2}, x^{2}-z^{2}\right)$. This map restricts to a pencil $f_{m}: U(\mathcal{A}) \rightarrow \mathbb{C P}^{1} \backslash\{(1,0),(0,1),(1,1)\}$, which yields another 2-dimensional component, $T=\left\{t \in\left(\mathbb{C}^{*}\right)^{6} \mid t_{1} t_{3} t_{6}=t_{1} t_{2}^{-1}=t_{3} t_{4}^{-1}=t_{5} t_{6}^{-1}=1\right\}$.

3.6. Translated tori. - In general, the characteristic variety $\mathcal{V}_{1}(\mathcal{A})$ also has irreducible components not passing through the origin. Following [14], we describe a combinatorial construction which, under certain assumptions, produces 1-dimensional translated subtori in $\mathcal{V}_{1}(\mathcal{A})$.

Fix a hyperplane $H \in \mathcal{A}$, and let $\mathcal{A}^{\prime}=\mathcal{A} \backslash\{H\}$ be the deletion of $\mathcal{A}$ with respect to $H$. A pointed multinet on $\mathcal{A}$ is a multinet $\mathcal{M}=\left(\left(\mathcal{A}_{1}, \ldots, \mathcal{A}_{k}\right), m, \mathcal{X}\right)$, together with a distinguished hyperplane $H \in \mathcal{A}$ for which $m_{H}>1$, and $m_{H} \mid n_{X}$ for each flat $X \in \mathcal{X}$ such that $X \geq H$.

Proposition 3.10 ([14]). - Suppose $\mathcal{A}$ admits a pointed multinet, and $\mathcal{A}^{\prime}$ is obtained from $\mathcal{A}$ by deleting the distinguished hyperplane $H$. Then $\mathcal{V}_{1}\left(\mathcal{A}^{\prime}\right)$ has a component which is a 1-dimensional subtorus of $\mathbb{T}\left(\mathcal{A}^{\prime}\right)$, translated by a character of order $m_{H}$ 
Proof. - Without loss of generality, we may assume that $H \in \mathcal{A}_{1}$. Consider the regular map given by $(26), f_{m}: M(\mathcal{A}) \rightarrow \mathbb{C P}^{1}$. Since $f_{H}$ does not divide $g_{2}$, we may extend $f_{m}$ to a regular map $\bar{f}_{m}: M\left(\mathcal{A}^{\prime}\right) \rightarrow \mathbb{C P}^{1}$. By construction, $f_{H} \mid g_{1}$, and so $\operatorname{im}\left(\bar{f}_{m}\right) \backslash \operatorname{im}\left(f_{m}\right)=\{[0: 1]\}$. Furthermore, $\operatorname{im}\left(\bar{f}_{m}\right)$ equals $U\left(\mathcal{P}_{1}\right)=\mathbb{C P}^{1} \backslash$ $\left\{[1: 0],\left[a_{3}: b_{3}\right]\right\}$. Passing to the projective complement, and taking the corestriction of $\bar{f}_{m}$ to its image yields an orbifold fibration, $\bar{f}_{m}: U\left(\mathcal{A}^{\prime}\right) \rightarrow U\left(\mathcal{P}_{1}\right)$.

By hypothesis, the degrees of the restrictions of $g_{1}$ and $g_{2}$ to the hyperplane $H$ are both divisible by $m_{H}$. Thus, the fiber of $\bar{f}_{m}$ over $[0: 1]$ has multiplicity $m_{H}$, and so $\bar{f}_{m}$ is a small pencil. The desired conclusion now follows from Theorem 3.8.

Example 3.11. - Let $\mathcal{A}$ be the $\mathrm{B}_{3}$ arrangement from Examples 2.9 and 2.13, and let $\mathcal{A}^{\prime}$ be the arrangement obtained by deleting the hyperplane $z=0$, as in Example 2.17. As noted in [59], the characteristic variety $\mathcal{V}_{1}\left(\mathcal{A}^{\prime}\right) \subset\left(\mathbb{C}^{*}\right)^{8}$ has 7 local components, corresponding to 6 triple points and one quadruple point, 5 components corresponding to braid sub-arrangements, and a component of the form $\rho T$, where $\rho=(1,1,-1,-1,-1,-1,1,1)$ and $T=\left\{\left(t^{2}, t^{-2}, 1,1, t^{-1}, t^{-1}, t, t\right) \mid t \in \mathbb{C}^{*}\right\}$.

To explain where this translated torus comes from, let $m$ be the multinet on $\mathcal{A}$ depicted in Figure 2, and let $Q_{m}(\mathcal{A})=g_{1} g_{2} g_{3}$ be the corresponding factorization of the defining polynomial of $(\mathcal{A}, m)$. We then obtain a (large) pencil $f_{m}: U(\mathcal{A}) \rightarrow$ $\mathbb{C P}^{1} \backslash\{[1: 0],[0: 1],[1: 1]\}$, given by $[x, y, z] \mapsto\left[z^{2}\left(x^{2}-y^{2}\right): y^{2}\left(x^{2}-z^{2}\right)\right]$.

Extending $f_{m}$ to the complement of $\mathcal{A}^{\prime}$ by allowing $z=0$ yields an orbifold fibration, $f_{m}: U\left(\mathcal{A}^{\prime}\right) \rightarrow \mathbb{P}^{1} \backslash\{[1: 0],[1: 1]\}$. Note that $f_{m}([x, y, 0])=\left[0: y^{2} x^{2}\right]$, and so the fiber over $[0: 1]$ has multiplicity 2 ; thus, $f_{m}$ is a small pencil. The orbifold fundamental group of the base of the pencil is $\mathbb{Z} * \mathbb{Z}_{2}$, and the pullback of $\mathcal{V}_{1}\left(\mathbb{Z} * \mathbb{Z}_{2}\right)=\mathbb{C}^{*} \times\{-1\}$ along $f_{m}$ yields the translated torus $\rho T \subset \mathcal{V}_{1}\left(\mathcal{A}^{\prime}\right)$.

Problem 3.12. - Do all positive-dimensional translated tori in the first characteristic variety of an arrangement arise in the manner described in Proposition 3.10?

Finally, there are also arrangements $\mathcal{A}$ with isolated torsion points in the characteristic variety $\mathcal{V}_{1}(\mathcal{A})$. Here is an example, also from [59].

Example 3.13. - Let $\mathcal{A}$ be the arrangement in $\mathbb{C}^{3}$ defined by the polynomial $Q(\mathcal{A})=x y z(y-x)(y+x)(2 y-z)(y-x-z)(y-x+z)(y+x+z)(y+x-z)$. Then $\mathcal{V}_{1}(\mathcal{A})$ has 10 local components, corresponding to 7 triple and 3 quadruple points, 17 components corresponding to braid sub-arrangements, 1 component corresponding to a $\mathrm{B}_{3}$ sub-arrangement, 3 translated components corresponding to deleted $\mathrm{B}_{3}$ sub-arrangements, and 2 isolated points of order 6 .

Problem 3.14. - Find a concrete description of the 0-dimensional components of the first characteristic variety $\mathcal{V}_{1}(\mathcal{A})$ of an arrangement $\mathcal{A}$. Are all such components determined by the intersection lattice $L(\mathcal{A})$ ?

Answering in the affirmative Problems 3.12 and 3.14 would lead to a solution (at least for $q=s=1$ ) of the following well-known problem, which is central to the theory of hyperplane arrangements. 
Problem 3.15. - Given a hyperplane arrangement $\mathcal{A}$, are the characteristic varieties $\mathcal{V}_{s}^{q}(M(\mathcal{A}), \mathbb{C})$ determined by the intersection lattice $L(\mathcal{A})$ ?

\section{The Milnor fibration of an arrangement. I.}

In this section, we introduce our main object of study: the Milnor fibration attached to a multi-arrangement. In the process, we describe several covering spaces related to the Milnor fiber.

4.1. The Milnor fibration. - Let $\mathcal{A}$ be an arrangement of hyperplanes in $\mathbb{C}^{d+1}$. Recall we associated to each multiplicity vector $m \in \mathbb{N}^{|\mathcal{A}|}$ a homogeneous polynomial

$$
Q_{m}(\mathcal{A})=\prod_{H \in \mathcal{A}} f_{H}^{m_{H}}
$$

of degree $N=\sum_{H \in \mathcal{A}} m_{H}$. Note that $Q_{m}(\mathcal{A})$ is a proper power if and only if $\operatorname{gcd}(m)>$ 1 , where $\operatorname{gcd}(m)=\operatorname{gcd}\left(m_{H}: H \in \mathcal{A}\right)$.

As before, let $M(\mathcal{A})$ be the complement of the arrangement. The polynomial map $Q_{m}(\mathcal{A}): \mathbb{C}^{d+1} \rightarrow \mathbb{C}$ restricts to a map $Q_{m}(\mathcal{A}): M(\mathcal{A}) \rightarrow \mathbb{C}^{*}$. As shown by J. Milnor [45] in a much more general context, $Q_{m}(\mathcal{A})$ is the projection map of a smooth, locally trivial bundle, known as the (global) Milnor fibration of the multi-arrangement $(\mathcal{A}, m)$. The typical fiber of this fibration,

$$
F_{m}(\mathcal{A})=Q_{m}(\mathcal{A})^{-1}(1)
$$

is called the Milnor fiber of the multi-arrangement, while the Milnor fibration itself is written as

$$
F_{m}(\mathcal{A}) \longrightarrow M(\mathcal{A}) \stackrel{Q_{m}(\mathcal{A})}{\longrightarrow} \mathbb{C}^{*} .
$$

Clearly, the Milnor fiber is a smooth manifold of dimension $2 d$. In fact, $F_{m}(\mathcal{A})$ is a Stein domain of complex dimension $d$, and thus has the homotopy type of a finite CW-complex of dimension $d$.

In the case when all the multiplicities $m_{H}$ are equal to 1 , the polynomial $Q(\mathcal{A})=$ $Q_{m}(\mathcal{A})$ is the usual defining polynomial for the arrangement, and has degree $n=|\mathcal{A}|$. Moreover, $F(\mathcal{A})=F_{m}(\mathcal{A})$ is the usual Milnor fiber of $\mathcal{A}$.

Remark 4.1. - Although the polynomials $Q_{m}(\mathcal{A})$ depend on the choice of multiplicities, they all have the same zero set; thus, they all share the same complement, namely, $M(\mathcal{A})$. On the other hand, the Milnor fibers $F_{m}(\mathcal{A})$ do depend on the various choices of $m$, and not just on $\mathcal{A}$. The next example illustrates this point.

Example 4.2. - Let $\mathcal{A}$ be the arrangement in $\mathbb{C}$ consisting of the single subspace $H=\{0\}$, and assign a multiplicity $m \in \mathbb{N}$ to that point. Then $Q_{m}(\mathcal{A})=z^{m}$, and so $F_{m}(\mathcal{A})$ is the set of $m$-roots of unity.

Of course, the Milnor fibers in the previous example are connected only when $m=1$. For an arbitrary arrangement $\mathcal{A}$, the number of connected components of $F_{m}(\mathcal{A})$ equals $\operatorname{gcd}(m)$. 

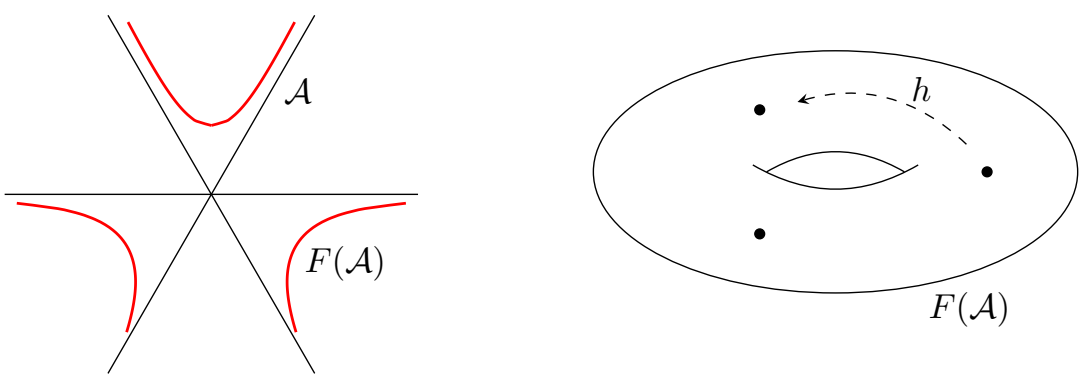

FiguRE 5. Milnor fiber and monodromy for $Q(\mathcal{A})=y\left(y^{2}-4 x^{2}\right)$

4.2. The geometric monodromy. - Consider the Milnor fibration $M(\mathcal{A}) \rightarrow \mathbb{C}^{*}$ defined by the polynomial $Q=Q_{m}(\mathcal{A})$. For each $\theta \in[0,1]$, let $F_{\theta}$ be the fiber over the point $e^{2 \pi \mathrm{i} \theta} \in \mathbb{C}^{*}$, so that $F_{0}=F_{1}=F_{m}(\mathcal{A})$.

For each $z \in M(\mathcal{A})$, the path $\gamma_{\theta, z}:[0,1] \rightarrow \mathbb{C}^{*}, t \mapsto e^{2 \pi \mathrm{i} t \theta}$ lifts to a path $\tilde{\gamma}_{\theta, z}:[0,1] \rightarrow M(\mathcal{A}), t \mapsto e^{2 \pi \mathrm{i} t \theta / N} z$, satisfying $\tilde{\gamma}_{\theta, z}(0)=z$. Notice that $Q\left(\tilde{\gamma}_{\theta, z}(1)\right)=$ $e^{2 \pi \mathrm{i} \theta} Q(z)$; thus, if $z \in F_{0}$, then $\tilde{\gamma}_{\theta, z}(1) \in F_{\theta}$.

By definition, the monodromy of the Milnor fibration is the diffeomorphism $h: F_{0} \rightarrow$ $F_{1}$ given by $h(z)=\tilde{\gamma}_{1, z}(1)$. In view of the above discussion, this diffeomorphism can be written as

$$
h: F_{m}(\mathcal{A}) \rightarrow F_{m}(\mathcal{A}), \quad z \mapsto e^{2 \pi \mathrm{i} / N} z .
$$

Clearly, the map $h$ has order $N$. Furthermore, note that the complement $M(\mathcal{A})$ is homotopy equivalent to the mapping torus of $h$ :

$$
M(\mathcal{A}) \simeq F_{m}(\mathcal{A}) \times[0,1] /(z, 0) \sim(h(z), 1) .
$$

Example 4.3. - Let $\mathcal{B}_{n}$ be the Boolean arrangement in $\mathbb{C}^{n}$, and identify the complement $M\left(\mathcal{B}_{n}\right)$ with the algebraic torus $\left(\mathbb{C}^{*}\right)^{n}$. Given a multiplicity vector $m$, the map $Q_{m}\left(\mathcal{B}_{n}\right):\left(\mathbb{C}^{*}\right)^{n} \rightarrow \mathbb{C}^{*}, z \mapsto z_{1}^{m_{1}} \cdots z_{n}^{m_{n}}$ is a morphism of algebraic groups. Hence, the Milnor fiber

$$
F_{m}\left(\mathcal{B}_{n}\right)=\operatorname{ker}\left(Q_{m}\left(\mathcal{B}_{n}\right)\right)
$$

is an algebraic subgroup, realized as the disjoint union of $\operatorname{gcd}(m)$ copies of $\left(\mathbb{C}^{*}\right)^{n-1}$. The monodromy automorphism, $h: F_{m}\left(\mathcal{B}_{n}\right) \rightarrow F_{m}\left(\mathcal{B}_{n}\right)$, permutes those copies in a circular fashion.

If $\operatorname{gcd}(m)=1$, the algebraic subgroup $F_{m}\left(\mathcal{B}_{n}\right)$ is, in fact, an algebraic torus isomorphic to $\left(\mathbb{C}^{*}\right)^{n-1}$. Moreover, the monodromy automorphism is isotopic to the identity, through the isotopy $h_{t}(z)=e^{2 \pi \mathrm{i} t / N} z$. Thus, the bundle $F_{m}\left(\mathcal{B}_{n}\right) \rightarrow M\left(\mathcal{B}_{n}\right) \rightarrow \mathbb{C}^{*}$ is trivial in this case.

4.3. Comparing two Milnor fibrations. - The previous example is, in some sense, a classifying object for Milnor fibrations of arrangements. 
To make this statement more precise, let $\mathcal{A}=\left\{H_{1}, \ldots, H_{n}\right\}$ be an (ordered) essential arrangement of hyperplanes in $\mathbb{C}^{d+1}$, with defining linear forms $f_{1}, \ldots, f_{n}$. Recall we defined in $\S 3.2$ a linear map $\iota(\mathcal{A}): \mathbb{C}^{d+1} \rightarrow \mathbb{C}^{n}$ by $\iota(\mathcal{A})(z)=\left(f_{1}(z), \ldots, f_{n}(z)\right)$. Restricting this map to the complement yields an embedding of $M(\mathcal{A})$ into $M\left(\mathcal{B}_{n}\right)=$ $\left(\mathbb{C}^{*}\right)^{n}$.

Since $\mathcal{A}$ and $\mathcal{B}_{n}$ have the same number of hyperplanes, a multiplicity vector for one of them yields a multiplicity vector for the other. The next lemma shows that the corresponding Milnor fibrations are compatible.

Lemma 4.4. - For each $m \in \mathbb{N}^{n}$, the map $\iota(\mathcal{A}): M(\mathcal{A}) \hookrightarrow M\left(\mathcal{B}_{n}\right)$ is compatible with the Milnor fibrations $Q_{m}(\mathcal{A}): M(\mathcal{A}) \rightarrow \mathbb{C}^{*}$ and $Q_{m}\left(\mathcal{B}_{n}\right): M\left(\mathcal{B}_{n}\right) \rightarrow \mathbb{C}^{*}$.

Proof. - Write $Q_{m}(\mathcal{A})=\prod_{i=1}^{n} f_{i}^{m_{i}}$ and $Q_{m}\left(\mathcal{B}_{n}\right)=\prod_{i=1}^{n} w_{i}^{m_{i}}$. Clearly, then, the following equality holds:

$$
Q_{m}(\mathcal{A})=Q_{m}\left(\mathcal{B}_{n}\right) \circ \iota(\mathcal{A}) .
$$

Thus, the map $\iota(\mathcal{A})$ restricts to an inclusion $\iota_{m}(\mathcal{A}): F_{m}(\mathcal{A}) \hookrightarrow F_{m}\left(\mathcal{B}_{n}\right)$, which fits into the commuting diagram

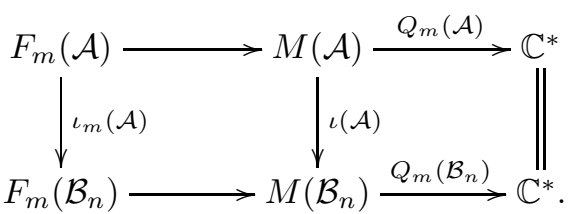

In other words, $\iota(\mathcal{A})$ is a bundle map, as claimed.

As a consequence of this lemma, we may expresses the Milnor fiber of $(\mathcal{A}, m)$ as the intersection of two familiar objects.

Corollary 4.5. - The Milnor fiber $F_{m}(\mathcal{A})$ is obtained by intersecting the complement $M(\mathcal{A})$, viewed as a subvariety of the algebraic torus $M\left(\mathcal{B}_{n}\right)=\left(\mathbb{C}^{*}\right)^{n}$ via the inclusion $\iota(\mathcal{A})$, with $F_{m}\left(\mathcal{B}_{n}\right) \cong \coprod_{\operatorname{gcd}(m)}\left(\mathbb{C}^{*}\right)^{n-1}$, viewed as an algebraic subgroup of $\left(\mathbb{C}^{*}\right)^{n}$ :

$$
F_{m}(\mathcal{A})=M(\mathcal{A}) \cap F_{m}\left(\mathcal{B}_{n}\right)
$$

4.4. Induced homomorphisms on $\pi_{1}$. - Using the above comparison between the two Milnor fibrations, we identify now the homomorphism induced on fundamental groups by the projection map $Q_{m}(\mathcal{A}): M(\mathcal{A}) \rightarrow \mathbb{C}^{*}$. As usual, denote by $\left\{x_{H} \mid H \in \mathcal{A}\right\}$ the standard generating set for $\pi_{1}(M(\mathcal{A}))$.

Proposition 4.6. - The induced homomorphism $Q_{m}(\mathcal{A})_{\sharp}: \pi_{1}(M(\mathcal{A})) \rightarrow \pi_{1}\left(\mathbb{C}^{*}\right)=$ $\mathbb{Z}$ is given by $x_{H} \mapsto m_{H}$.

Proof. - From $(34)$, we know that $Q_{m}(\mathcal{A})=Q_{m}\left(\mathcal{B}_{n}\right) \circ \iota(\mathcal{A})$. By Lemma 3.3, the homomorphism $\iota(\mathcal{A})_{\sharp}: \pi_{1}(M(\mathcal{A})) \rightarrow \pi_{1}\left(\left(\mathbb{C}^{*}\right)^{n}\right)$ may be identified with the abelianization $\operatorname{map}, \mathrm{ab}: \pi_{1}(M(\mathcal{A})) \rightarrow \mathbb{Z}^{n}$. 
On the other hand, the homomorphism induced in $\pi_{1}$ by the algebraic morphism $Q_{m}\left(\mathcal{B}_{n}\right):\left(\mathbb{C}^{*}\right)^{n} \rightarrow \mathbb{C}^{*}, w \mapsto w_{1}^{m_{1}} \cdots w_{n}^{m_{n}}$ may be identified with the linear map $m: \mathbb{Z}^{n} \rightarrow \mathbb{Z}, v \mapsto \sum_{i=1}^{n} m_{i} v_{i}$. The conclusion follows.

Using this proposition, we can derive in a novel way a well-known lower-bound on the first Betti number of the Milnor fiber of a (multi-) arrangement.

Corollary 4.7. - Suppose $\operatorname{gcd}(m)=1$. Then $b_{1}\left(F_{m}(\mathcal{A})\right) \geq n-1$, where $n=|\mathcal{A}|$.

Proof. - From the assumption, we know that the Milnor fibers $F_{m}(\mathcal{A})$ and $F_{m}\left(\mathcal{B}_{n}\right)$ are connected. Applying the $\pi_{1}$ functor to diagram (35), and using the homotopy long exact sequences associated to the respective Milnor fibrations, as well as Lemma 3.3 and Proposition 4.6, we obtain the following commuting diagram with exact rows:

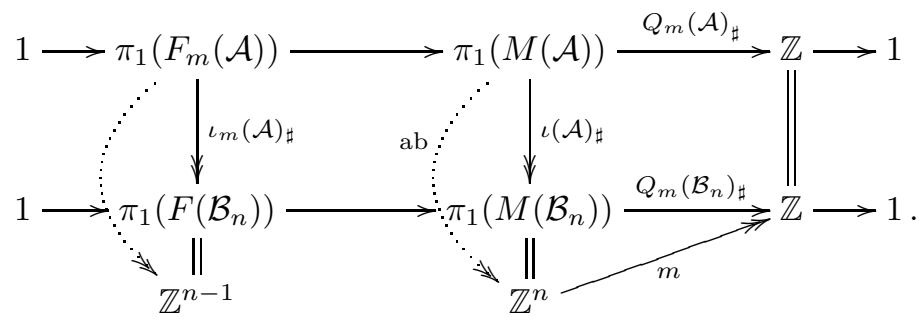

This diagram yields an epimorphism $\pi_{1}\left(F_{m}(\mathcal{A})\right) \rightarrow \mathbb{Z}^{n-1}$, indicated by a dotted arrow in (36). This completes the proof.

We will come back to this topic in $\S 5$, where we will use a different approach to provide improved estimates on the Betti numbers of the Milnor fiber.

4.5. The Milnor fiber as an infinite cyclic cover. - A standard construction turns any continuous map into a fibration, at least up to homotopy, see e.g. [27, §4.3]. In the case of an inclusion $F \hookrightarrow E$, the construction is particularly simple: let $Y$ be the space of paths in $E$ starting in $F$. Then the inclusion $F \hookrightarrow Y, a \mapsto$ const $_{a}$ is a homotopy equivalence, while the map $g: Y \rightarrow E, \gamma \mapsto \gamma(1)$ is a fibration.

Now suppose $F \rightarrow E \stackrel{p}{\rightarrow} B$ is a fibration, and all three spaces are path-connected $\mathrm{CW}$-complexes. Pick a basepoint $e_{0} \in E$, and let $Z:=g^{-1}\left(e_{0}\right)$ be the homotopy fiber of the inclusion $F \hookrightarrow E$. Let $\Omega B$ be the space of loops in $B$, based at $b_{0}=p\left(e_{0}\right)$. Then $\Omega B$ has the homotopy type of a CW-complex, and we have a map $f: \Omega B \rightarrow Z$, which sends a loop at $b_{0}$ to its lift at $e_{0}$, traversed in the opposite direction. Comparing the long exact sequences of homotopy groups of the two fibrations, $F \rightarrow E \rightarrow B$ and $Z \rightarrow Y \rightarrow E$, we conclude that $f$ is a homotopy equivalence.

In our situation, consider the Milnor fibration (29) associated to $(\mathcal{A}, m)$. The homotopy fiber of the inclusion $F_{m}(\mathcal{A}) \rightarrow M(\mathcal{A})$, then, is homotopic to $\Omega \mathbb{C}^{*} \simeq \mathbb{Z}$.

Proposition 4.8. - The homotopy fibration defined by the inclusion $F_{m}(\mathcal{A}) \hookrightarrow$ $M(\mathcal{A})$ is equivalent to the regular, infinite cyclic cover classified by the homomorphism $\pi_{1}(M(\mathcal{A})) \rightarrow \mathbb{Z}, x_{H} \mapsto m_{H}$. 
Proof. - The exponential map exp: $\mathbb{C} \rightarrow \mathbb{C}^{*}, \zeta \mapsto e^{2 \pi \mathrm{i} \zeta}$ is a regular $\mathbb{Z}$-cover. Pulling back this cover along the map $Q=Q_{m}(\mathcal{A}): M(\mathcal{A}) \rightarrow \mathbb{C}^{*}$, we obtain the diagram

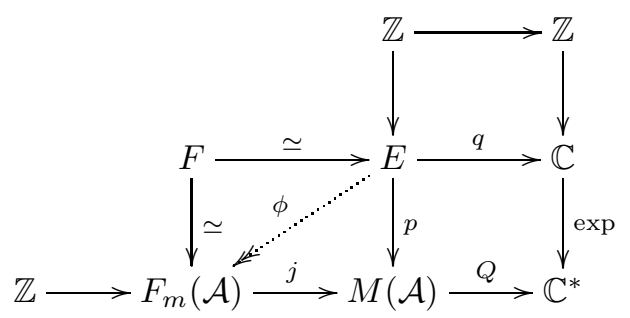

where $F$ is the homotopy fiber of $q$, the homotopy equivalence $F \rightarrow F_{m}(\mathcal{A})$ is the restriction of $p$ to (homotopy) fibers, and $\Omega \mathbb{C}^{*} \simeq \mathbb{Z}$ is the homotopy fiber of $j$.

By construction, the pullback cover, $E \rightarrow M(\mathcal{A})$, is a regular $\mathbb{Z}$-cover, classified by the homomorphism $Q_{\sharp}: \pi_{1}(M(\mathcal{A})) \rightarrow \mathbb{Z}$. Furthermore, the homotopy equivalence $\phi: E \stackrel{\simeq}{\longrightarrow} F_{m}(\mathcal{A})$ defined by diagram (37) satisfies $j \circ \phi \simeq p$. Thus, the cover $E \rightarrow M(\mathcal{A})$ may be identified with the homotopy fibration associated to the inclusion $F_{m}(\mathcal{A}) \rightarrow M(\mathcal{A})$. The desired conclusion now follows from Proposition 4.6.

Remark 4.9. - In general, an infinite cyclic cover of a finite CW-complex need not have finite Betti numbers, let alone have the homotopy type of a finite $\mathrm{CW}$ complex. For instance, if $X=S^{1} \vee S^{1}$ is a wedge of two circles, and $Y \rightarrow X$ is an arbitrary $\mathbb{Z}$-cover, then $b_{1}(Y)=\infty$. In our situation, though, the geometry of the situation conspires to insure that all the $\mathbb{Z}$-covers corresponding to Milnor fibrations of arrangements have finite cell decompositions. For more on the geometric and homological finiteness properties of (infinite) abelian covers, we refer to [61, 62, 63].

4.6. The Milnor fiber as a finite cyclic cover. - As before, fix a multiplicity vector $m$ on $\mathcal{A}$ with $\operatorname{gcd}(m)=1$, and set $N=\sum_{H \in \mathcal{A}} m_{H}$. The monodromy automorphism $h: F_{m}(\mathcal{A}) \rightarrow F_{m}(\mathcal{A})$, given by $h(z)=e^{2 \pi \mathrm{i} / N} z$, generates a cyclic group $\mathbb{Z}_{N}$. As noted in [47], this group acts freely on $F_{m}(\mathcal{A})$, and the quotient space may be identified with the projective complement, $U(\mathcal{A})$. We thus have a regular, $N$-fold cyclic cover, $F_{m}(\mathcal{A}) \rightarrow U(\mathcal{A})$. As shown in $[8,6]$, this cover can be described in purely group-theoretic terms.

We give here a self-contained, full-detail proof of this basic result. The proof uses a somewhat different approach, which draws in part from [60, 14]. To start with, recall from $\S 2.2$ that the Hopf fibration $\pi: \mathbb{C}^{d+1} \backslash\{0\} \rightarrow \mathbb{C P}^{d}$ restricts to a (trivializable) bundle map, $\pi(\mathcal{A}): M(\mathcal{A}) \rightarrow U(\mathcal{A})$, with fiber $\mathbb{C}^{*}$.

Theorem 4.10. - The map $\pi(\mathcal{A}): M(\mathcal{A}) \rightarrow U(\mathcal{A})$ restricts to a regular, $\mathbb{Z}_{N}$-cover $\pi_{m}(\mathcal{A}): F_{m}(\mathcal{A}) \rightarrow U(\mathcal{A})$. Furthermore, this cover is classified by the homomorphism

$$
\delta_{m}: \pi_{1}(U(\mathcal{A})) \rightarrow \mathbb{Z}_{N}, \quad \bar{x}_{H} \mapsto m_{H} \bmod N .
$$

Proof. - For simplicity, we will drop the arrangement $\mathcal{A}$ from the notation. By homogeneity of the polynomial $Q_{m}$, we have that $Q_{m}(w z)=w^{N} Q_{m}(z)$, for every 
$z \in M$ and $w \in \mathbb{C}^{*}$. Thus, the restriction of $Q_{m}$ to a fiber of $\pi$ may be identified with the covering projection $q: \mathbb{C}^{*} \rightarrow \mathbb{C}^{*}, q(w)=w^{N}$.

Now, if both $z$ and $w z$ belong to $F_{m}$, then $Q_{m}(z)=Q_{m}(w z)=1$, and so $w^{N}=1$. Thus, the restriction $\pi_{m}=\left.\pi\right|_{F_{m}}: F_{m} \rightarrow U$ is the orbit map of the free action of the geometric monodromy on $F_{m}$. Hence, $\pi_{m}$ is a regular $\mathbb{Z}_{N}$-cover, as claimed.

To determine the classifying homomorphism $\delta_{m}: \pi_{1}(U) \rightarrow \mathbb{Z}_{N}$ for this cover, we will make some auxiliary constructions. We refer to diagram (38) for the various spaces and maps introduced along the way.

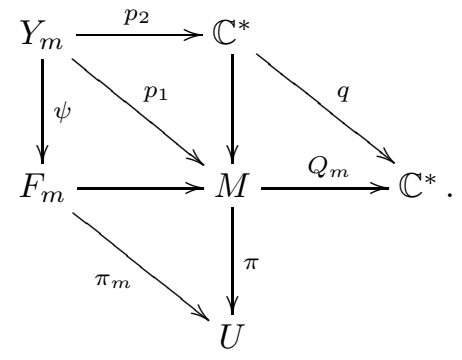

Let $Y_{m}=\left\{(z, w) \in M \times \mathbb{C}^{*} \mid Q_{m}(z)=w^{N}\right\}$, and denote by $p_{1}: Y_{m} \rightarrow M$ and $p_{2}: Y_{m} \rightarrow \mathbb{C}^{*}$ the restrictions of the coordinate projections. Clearly,

$$
Q_{m} \circ p_{1}=q \circ p_{2} .
$$

Thus, $p_{1}$ is a regular $\mathbb{Z}_{N}$-cover, obtained as the pullback of $q$ along $Q_{m}$. Of course, the cover $q$ is classified by the canonical projection $\pi_{1}\left(\mathbb{C}^{*}\right)=\mathbb{Z} \rightarrow \mathbb{Z}_{N}$. Hence, by Proposition 4.6, the cover $p_{1}$ is classified by the homomorphism

$$
\pi_{1}(M) \rightarrow \mathbb{Z}_{N}, \quad x_{H} \mapsto m_{H} \bmod N .
$$

We now proceed to the other side of diagram (38). Given an element $(z, w) \in Y_{m}$, note that $Q_{m}\left(w^{-1} z\right)=w^{-N} Q_{m}(z)=1$. Hence, we may define a map $\psi: Y_{m} \rightarrow F_{m}$ by $\psi(z, w)=w^{-1} z$. Plainly,

$$
\pi_{m} \circ \psi=\pi \circ p_{1} .
$$

Therefore, the pullback of $\pi_{m}$ along $\pi$ coincides with the cover $p_{1}$. Now, by Lemma 3.3 , the homomorphism $\left.\pi_{\sharp}: \pi_{1}(M) \rightarrow \pi_{1} U\right)$ is given by $x_{H} \mapsto \bar{x}_{H}$. Thus, the cover $p_{1}$ is classified by the homomorphism

$$
\pi_{1}(M) \rightarrow \mathbb{Z}_{N}, \quad x_{H} \mapsto \delta_{m}\left(\bar{x}_{H}\right) .
$$

Comparing the answers obtained in (40) and (42), we find that $\delta_{m}\left(\bar{x}_{H}\right)=m_{H} \bmod$ $N$, and we are done.

\section{The Milnor fibration of an arrangement. II.}

We continue our discussion of the Milnor fibration with a study of its homology groups and monodromy operators. 
5.1. Homology of the Milnor fiber. - Using the interpretation of the Milnor fiber of a multi-arrangement as a finite cyclic cover of the projectivized complement, we may compute the homology groups of the Milnor fiber and the characteristic polynomial of the algebraic monodromy in each degree, at least over a field of characteristic not dividing the order of the cover.

Theorem 5.1. - Let $(\mathcal{A}, m)$ be a multi-arrangement. Set $N=\sum_{H \in \mathcal{A}} m_{H}$, and let $\delta_{m}: \pi_{1}(U(\mathcal{A})) \rightarrow \mathbb{Z}_{N}$ be the homomorphism given by $x_{H} \mapsto m_{H} \bmod N$. If $\mathbb{k}$ is a coefficient field of characteristic not dividing $N$, then

1. The homology groups of the Milnor fiber $F_{m}(\mathcal{A})$ are given by

$$
\operatorname{dim}_{\mathbb{k}} H_{q}\left(F_{m}(\mathcal{A}), \mathbb{k}\right)=\sum_{s \geq 1}\left|\mathcal{V}_{s}^{q}(U(\mathcal{A}), \mathbb{k}) \cap \operatorname{im}\left(\widehat{\delta_{m}}\right)\right| .
$$

2. The characteristic polynomial of the algebraic monodromy of the Milnor fibration, $h_{*}: H_{q}\left(F_{m}(\mathcal{A}), \mathbb{k}\right) \rightarrow H_{q}\left(F_{m}(\mathcal{A}), \mathbb{k}\right)$, is given by

$$
\Delta_{h, q}^{\mathrm{k}}(t)=\prod_{s \geq 1} \prod_{\substack{\zeta \in \mathbb{k}^{*}: \zeta^{N}=1, \zeta^{m} \in \mathcal{V}_{s}^{q}(U(\mathcal{A}), \mathbf{k})}}(t-\zeta) .
$$

Proof. - By Theorem 4.10, the restriction of the Hopf fibration to the Milnor fiber, $\pi_{m}(\mathcal{A}): F_{m}(\mathcal{A}) \rightarrow U(\mathcal{A})$, is a regular, $\mathbb{Z}_{N}$-cover classified by the homomorphism $\delta_{m}$. The desired conclusions follow from Theorem B.1.

Remark 5.2. - The 1-eigenspace of the linear transformation $h_{*}$ acting on the $\mathbb{k}$-vector space $H_{q}\left(F_{m}(\mathcal{A}), \mathbb{k}\right)$ is isomorphic to $H_{q}(U(\mathcal{A}), \mathbb{k})$, which has dimension $b_{q}(U(\mathcal{A}))$, independent of $\mathbb{k}$. In particular, $h_{*}$ is the identity on $H_{q}\left(F_{m}(\mathcal{A}), \mathbb{k}\right)$ if and only if $\Delta_{h, q}^{\mathrm{k}}(t)=(t-1)^{b_{q}(U(\mathcal{A}))}$. The other eigenvalues of $h_{*}$, and the dimensions of their eigenspaces, depend in principle on both the multiplicity vector $m$ and the characteristic of $\mathbb{k}$.

Remark 5.3. - In the case when all multiplicities $m_{H}$ are equal to 1 , we can say more: in the product (44), only non-primitive roots of 1 appear, provided $\mathbb{k}=\mathbb{C}$ and $q<d$, see [8, Corollary 2.2]. For more refined versions of this result, we refer to [37, Proposition 2.1] and [41, Theorem 3.13].

Example 5.4. - Let $\mathcal{P}_{n}$ be the pencil of $n+1$ lines through the origin of $\mathbb{C}^{2}$ from Examples 2.3 and 3.6. We know that the Milnor fiber $F_{m}\left(\mathcal{P}_{n}\right)$ is an $N$-fold cover of $U\left(\mathcal{P}_{n}\right)=\mathbb{C} \backslash\{n$ points $\}$, where $N$ is the sum of the multiplicities. We also know that $F_{m}\left(\mathcal{P}_{n}\right)$ is a Riemann surface with $N$ punctures. A standard Euler characteristic argument now shows that the genus of this surface is $g=\frac{N(n-2)}{2}+1$; thus,

$$
\operatorname{dim}_{\mathbb{k}} H_{1}\left(F_{m}\left(\mathcal{P}_{n}\right), \mathbb{k}\right)=N(n-1)+1,
$$

for all fields $\mathbb{k}$.

Alternatively, we know from $(22)$ that $\mathcal{V}_{1}^{1}\left(U\left(\mathcal{P}_{n}\right), \mathbb{k}\right)=\cdots=\mathcal{V}_{n-1}^{1}\left(U\left(\mathcal{P}_{n}\right), \mathbb{k}\right)=$ $\left(\mathbb{k}^{*}\right)^{n}$ and $\mathcal{V}_{n}^{1}\left(U\left(\mathcal{P}_{n}\right), \mathbb{k}\right)=\{1\}$. Thus, we may recover the calculation from (45) by means of formula (43), at least in the case when $\operatorname{char}(\mathbb{k}) \nmid N$. 
In view of Theorem 3.8, the above result yields a rather explicit formula for the first Betti number of the Milnor fiber $F(\mathcal{A})$, and for the characteristic polynomial $\Delta=\Delta_{h, 1}^{\mathbb{C}}$ of its algebraic monodromy, $h_{*}: H_{1}(F(\mathcal{A}), \mathbb{C}) \rightarrow H_{1}(F(\mathcal{A}), \mathbb{C})$. Let $\Phi_{r}$ be the $r$-th cyclotomic polynomial, and let $\varphi(r)$ be its degree.

Corollary 5.5. - Let $\mathcal{A}$ be an arrangement of $n$ hyperplanes, and let $F(\mathcal{A})$ be its Milnor fiber. Then,

$$
\Delta(t)=(t-1)^{n-1} \cdot \prod_{1 \neq r \mid n} \Phi_{r}(t)^{\operatorname{depth}\left(\delta^{n / r}\right)},
$$

where $\delta: \pi_{1}(U(\mathcal{A})) \rightarrow \mathbb{C}^{*}$ is the "diagonal" character, given by $\delta\left(x_{H}\right)=e^{2 \pi \mathrm{i} / n}$, for all $H \in \mathcal{A}$, and $\operatorname{depth}(\rho):=\max \left\{s \mid \rho \in \mathcal{V}_{s}^{1}(U(\mathcal{A}))\right\}$. In particular,

$$
b_{1}(F(\mathcal{A}))=n-1+\sum_{1 \neq r \mid n} \varphi(r) \operatorname{depth}\left(\delta^{n / r}\right),
$$

Remark 5.6. - In formula (47), only the essential components of $\mathcal{V}_{1}(\mathcal{A})$ may contribute to the sum. Indeed, if a component lies on a (proper) coordinate subtorus $C \subset \mathbb{T}(\mathcal{A})$, then the diagonal subtorus, $D=\left\{(t, \ldots, t) \mid t \in \mathbb{C}^{*}\right\}$, intersects $C$ only at the origin. In particular, local components, or, more generally, components arising from multinets supported on proper sub-arrangements of $\mathcal{A}$, do not produce jumps in the first Betti number of $F(\mathcal{A})$.

On the other hand, if $(\mathcal{A}, m)$ is a multi-arrangement with some $m_{H}>1$, then even the non-essential components in the characteristic varieties of $U(\mathcal{A})$ may produce jumps in the Betti numbers of $F_{m}(\mathcal{A})$, according to formula (43).

Remark 5.7. - In [7, Theorem 13], Cohen, Dimca, and Orlik give some nice combinatorial upper bounds on the exponents of the cyclotomic polynomials appearing in formula (46). For more on this, we refer to $[16, \S 6.4]$.

5.2. Discussion an examples. - We now illustrate with a few examples the range of applicability of Theorem 5.1 (see [8] for more computations of this sort.)

Example 5.8. - Let $\mathcal{A}$ be the braid arrangement from Examples 2.8, 2.12, and 3.9. We know that $\mathcal{V}_{1}(\mathcal{A})$ has a single essential component, namely, the subtorus $T=\left\{t \in\left(\mathbb{C}^{*}\right)^{6} \mid t_{1} t_{3} t_{6}=t_{1} t_{2}^{-1}=t_{3} t_{4}^{-1}=t_{5} t_{6}^{-1}=1\right\}$. Clearly, $\delta^{2} \in T$, yet $\delta \notin T$; hence, $\Delta(t)=(t-1)^{5}\left(t^{2}+t+1\right)$.

Example 5.9. - Let $\mathcal{A}$ be the reflection arrangement of type $\mathrm{B}_{3}$ from Examples 2.9 and 2.13. We know that $\mathcal{V}_{1}(\mathcal{A})$ has an essential component, corresponding to the $(3,4)$-multinet depicted in Figure 2. It is readily verified that this is the only such component, and that the diagonal subtorus intersects this component only at the origin. Hence, $\Delta(t)=(t-1)^{8}$.

Example 5.10. - Let $\mathcal{A}$ be the Ceva(3) arrangement from Example 2.14. The $(3,3)$-net depicted in Figure 3 defines a rational map, $\mathbb{C P}^{2} \rightarrow \mathbb{C P}^{1},(x, y, z) \mapsto$ $\left(x^{3}-y^{3}, y^{3}-z^{3}\right)$, which restricts to a pencil $U(\mathcal{A}) \rightarrow \mathbb{C P}^{1} \backslash\{(1,0),(0,1),(1,-1)\}$. Let $T$ be the essential 2-dimensional component of $\mathcal{V}_{1}(\mathcal{A})$ obtained by pullback along this 
pencil. It is readily verified that the subgroup generated by the diagonal character $\delta$ intersects $\mathcal{V}_{2}(\mathcal{A})$ in two points, both lying on $T$, and both of order 3 . Hence, $\Delta(t)=(1-t)^{8}\left(1+t+t^{2}\right)^{2}$.

As the next example shows, the first Betti number of the Milnor fiber of an arrangement in $\mathbb{C}^{3}$ does not only depend on the number and type of multiple points of $\mathbb{P}(\mathcal{A})$, but also on their relative position.

Example 5.11. - Consider the arrangements $\mathcal{A}_{1}$ and $\mathcal{A}_{2}$ defined by the polynomials

$$
\begin{aligned}
& Q\left(\mathcal{A}_{1}\right)=x y z(x-y)(y-z)(x-y-z)(2 x+y+z)(2 x+y-z)(2 x-5 y+z), \\
& Q\left(\mathcal{A}_{2}\right)=x y z(x+y)(y+z)(x+3 z)(x+2 y+z)(x+2 y+3 z)(2 x+3 y+3 z) .
\end{aligned}
$$

The arrangement $\mathcal{A}_{1}$ is a realization of the Pappus configuration $\left(9_{3}\right)_{1}$, while $\mathcal{A}_{2}$ is a realization of the configuration $\left(9_{3}\right)_{2}$. Each projective configurations has 9 double points and 9 triple points; additionally, the first configuration supports a $(3,3)$-net, but the second one supports no essential multinet. Applying formula (46), we find that $\Delta_{1}(t)=(t-1)^{8}\left(t^{2}+t+1\right)$ and $\Delta_{2}(t)=(t-1)^{8}$, as in [8].

Such examples, and many others from the literature (see for instance [41, 4, 39, $18,65]$ for some recent progress in this direction) raise the following well-known question, dating from the 1980s.

Problem 5.12. - Given an arrangement $\mathcal{A}$, are the Betti numbers of the Milnor fiber $F(\mathcal{A})$ and the characteristic polynomial of the algebraic monodromy determined by the intersection lattice $L(\mathcal{A})$ ?

5.3. Torsion in the homology of the Milnor fiber. - A long-standing question, raised by Randell and Dimca-Némethi among others, asks whether the Milnor fiber of an arrangement can have non-trivial torsion in homology. Examples from [6] first showed that $H_{1}\left(F_{m}(\mathcal{A}), \mathbb{Z}\right)$ may have torsion, for suitable multi-arrangements $(\mathcal{A}, m)$. In [14], these examples were recast in a more general framework, leading to examples of high-dimensional hyperplane arrangements $\mathcal{A}$ for which $H_{q}(F(\mathcal{A}), \mathbb{Z})$ has torsion, for some $q>1$. Let us sketch here this circle of ideas.

Theorem 5.13 ([14]). - Suppose $\mathcal{A}$ admits a pointed multinet, with distinguished hyperplane $H$ and multiplicity $m$. Let $p$ be a prime dividing $m_{H}$. There is then a choice of multiplicities $m^{\prime}$ on the deletion $\mathcal{A}^{\prime}=\mathcal{A} \backslash\{H\}$ such that $H_{1}\left(F_{m^{\prime}}\left(\mathcal{A}^{\prime}\right), \mathbb{Z}\right)$ has non-zero p-torsion.

Proof. - (Sketch) By Proposition 3.10, the variety $\mathcal{V}_{1}\left(\mathcal{A}^{\prime}\right)$ has a component of the form $\rho T$, where $T$ is a 1-dimensional subtorus, and $\rho$ is a torsion character, of order divisible by $p$. Using this fact, and some further machinery, it is shown in [14, Theorem 6.1], that, for all sufficiently large integers $r>1$ not divisible by $p$, there exists a regular, $r$-fold cyclic cover $Y \rightarrow U\left(\mathcal{A}^{\prime}\right)$ such that $H_{1}(Y, \mathbb{Z})$ has $p$-torsion.

On the other hand, it is also shown in [14, Proposition 6.7] that any finite cyclic cover of an arrangement complement is dominated by a Milnor fiber corresponding to a suitable choice of multiplicities. Hence, there exists a choice of multiplicities $m^{\prime}$ for 
which the cover $\pi_{m^{\prime}}\left(\mathcal{A}^{\prime}\right): F_{m^{\prime}}\left(\mathcal{A}^{\prime}\right) \rightarrow U\left(\mathcal{A}^{\prime}\right)$ factors through $Y$, and $p$ does not divide $N^{\prime}:=\sum_{H \in \mathcal{A}^{\prime}} m_{H}^{\prime}$. A standard transfer argument then shows that $H_{1}\left(F_{m^{\prime}}\left(\mathcal{A}^{\prime}\right), \mathbb{Z}\right)$ also has $p$-torsion.

Example 5.14. - Let $\mathcal{A}^{\prime}$ be the deletion of the $\mathrm{B}_{3}$ arrangement from Example 3.11. Proceeding as above, let $r=3$ and consider the 3 -fold cover $Y \rightarrow U\left(\mathcal{A}^{\prime}\right)$ classified by the vector $\chi=(2,1,0,0,2,2,1,1) \in\left(\mathbb{Z}_{3}\right)^{8}$. Choose $m^{\prime}=(2,1,3,3,2,2,1,1)$ as a positive integer lift of $\chi$. Then $N^{\prime}=15$, and $F_{m^{\prime}}\left(\mathcal{A}^{\prime}\right)$ factors through $Y$; thus, there is 2-torsion in the first homology of $F_{m^{\prime}}\left(\mathcal{A}^{\prime}\right)$. Explicit calculation shows that, in fact, $H_{1}\left(F_{m^{\prime}}\left(\mathcal{A}^{\prime}\right), \mathbb{Z}\right)=\mathbb{Z}^{7} \oplus \mathbb{Z}_{2} \oplus \mathbb{Z}_{2}$ and $\Delta_{1}^{\overline{F_{2}}}(t)=(t-1)^{7}\left(t^{2}+t+1\right)$.

This leads to a couple of natural questions (see also $[6,14]$ ).

Problem 5.15. - Is there a hyperplane arrangement $\mathcal{A}$ (without multiplicities) such that $H_{1}(F(\mathcal{A}), \mathbb{Z})$ has non-trivial torsion?

Problem 5.16. - Given a hyperplane arrangement $\mathcal{A}$, is the torsion in $H_{*}(F(\mathcal{A}), \mathbb{Z})$ determined by the intersection lattice $L(\mathcal{A})$ ?

5.4. A polarization construction. - Given a multi-arrangement $(\mathcal{A}, m)$, we define in [14] a new hyperplane arrangement, $\mathcal{B}=\mathcal{A} \| m$, called the polarization of the multi-arrangement. The rank of $\mathcal{B}$ equals $\operatorname{rank} \mathcal{A}+\left|\left\{H \in \mathcal{A}: m_{H} \geq 2\right\}\right|$, while the number of hyperplanes in $\mathcal{B}$ equals $N=\sum_{H \in \mathcal{A}} m_{H}$.

A crucial point of this construction is the connection between the Milnor fiber of the (simple) arrangement $\mathcal{B}$ and the Milnor fiber of the multi-arrangement $(\mathcal{A}, m)$ : the pullback of the cover $F(\mathcal{B}) \rightarrow U(\mathcal{B})$ along the canonical inclusion $U(\mathcal{A}) \rightarrow U(\mathcal{B})$ is equivalent to the cover $F_{m}(\mathcal{A}) \rightarrow U(\mathcal{A})$. Using this fact, together with Theorem 5.13 , the following result is proved in [14].

Theorem 5.17 ([14]). — Suppose $\mathcal{A}$ admits a pointed multinet, with distinguished hyperplane $H$ and multiplicity $m$. Let $p$ be a prime dividing $m_{H}$. There is then a choice of multiplicities $m^{\prime}$ on the deletion $\mathcal{A}^{\prime}=\mathcal{A} \backslash\{H\}$ such that $H_{q}(F(\mathcal{B}), \mathbb{Z})$ has p-torsion, where $\mathcal{B}=\mathcal{A}^{\prime} \| m^{\prime}$ and $q=1+\left|\left\{K \in \mathcal{A}^{\prime}: m_{K}^{\prime} \geq 3\right\}\right|$.

Example 5.18. - Let $\mathcal{A}^{\prime}$ be the deleted $B_{3}$ arrangement from Examples 3.11 and 5.14. Then the choice of multiplicities $m^{\prime}=(8,1,3,3,5,5,1,1)$ produces an arrangement $\mathcal{B}=\mathcal{A}^{\prime} \| m^{\prime}$ of 27 hyperplanes in $\mathbb{C}^{8}$, such that $H_{6}(F(\mathcal{B}), \mathbb{Z})$ has 2 -torsion of rank 108 .

5.5. Characteristic varieties of the Milnor fiber. - Very little is known about the homology with coefficients in rank 1 local systems of the Milnor fiber of a (multi-) arrangement $(\mathcal{A}, m)$. Since $F_{m}(\mathcal{A})$ is a smooth, quasi-projective variety, deep theorems of Arapura [1] and Budur-Wang [5] guarantee that the characteristic varieties $\mathcal{V}_{s}^{q}\left(F_{m}(\mathcal{A}), \mathbb{C}\right)$ are unions of torsion-translated subtori. Let us analyze in more detail these varieties, following the approach of Dimca and Papadima from [19].

As in $\S 4.6$, let $\pi=\pi_{m}(\mathcal{A}): F_{m}(\mathcal{A}) \rightarrow U(\mathcal{A})$ be the restriction of the Hopf fibration to the Milnor fiber, and let $h: F_{m}(\mathcal{A}) \rightarrow F_{m}(\mathcal{A})$ be the monodromy of the Milnor fibration. Since $\pi$ is a finite, regular cover, Proposition B.3 guarantees that 
$\pi^{*}\left(\mathcal{V}_{s}^{q}(U(\mathcal{A}), \mathbb{k})\right) \subseteq \mathcal{V}_{s}^{q}\left(F_{m}(\mathcal{A}), \mathbb{k}\right)$ and $\pi^{*}\left(\mathcal{R}_{s}^{q}(U(\mathcal{A}), \mathbb{k})\right) \subseteq \mathcal{R}_{s}^{q}\left(F_{m}(\mathcal{A}), \mathbb{k}\right)$, for all $q \geq 0$ and $s \geq 1$.

If $h_{*}$ acts as the identity on $H_{1}\left(F_{m}(\mathcal{A}), \mathbb{k}\right)$, more can be said. Indeed, Corollary B.4 implies that $\pi^{*}: \mathcal{R}_{s}^{1}(U(\mathcal{A}), \mathbb{k}) \rightarrow \mathcal{R}_{s}^{1}\left(F_{m}(\mathcal{A}), \mathbb{k}\right)$ is an isomorphism, for all $s \geq$ 1. Furthermore, by Theorem C.4, the map $\pi^{*}: H^{1}\left(U(\mathcal{A}), \mathbb{C}^{*}\right) \rightarrow H^{1}\left(F_{m}(\mathcal{A}), \mathbb{C}^{*}\right)$ restricts to an isomorphism between the components through the identity of $\mathcal{V}_{s}(\mathcal{A})$ and $\mathcal{V}_{s}^{1}\left(F_{m}(\mathcal{A}), \mathbb{C}\right)$.

Let us restrict now our attention to the usual Milnor fiber, $F(\mathcal{A})$, and its degree 1 characteristic varieties, $\mathcal{V}_{s}(F(\mathcal{A}))=\mathcal{V}_{s}^{1}(F(\mathcal{A}), \mathbb{C})$. In general, the inclusion $\pi^{*}: \mathcal{V}_{s}(\mathcal{A}) \hookrightarrow \mathcal{V}_{s}(F(\mathcal{A}))$ is strict. For instance, suppose $\mathcal{A}$ admits a non-trivial, reduced multinet $\mathcal{M}$, and let $T_{\mathcal{M}}$ be the corresponding component of $\mathcal{V}_{1}(\mathcal{A})$. It is then shown in [19] that $\mathcal{V}_{1}(F(\mathcal{A}))$ has a component passing through the identity and containing $\pi^{*}\left(T_{\mathcal{M}}\right)$ as a proper subset.

Example 5.19. - Let $\mathcal{A}$ be the braid arrangement from Figure 1. Recall from Examples 3.9 and 5.8 that $\mathcal{V}_{1}(\mathcal{A})$ has four local components, $T_{1}, \ldots, T_{4}$, corresponding to the triple points, and an essential component $T$, corresponding to a $(3,2)$-net. By the above discussion, the characteristic variety $\mathcal{V}_{1}(F(\mathcal{A})) \subset\left(\mathbb{C}^{*}\right)^{7}$ has 2 -dimensional components $\pi^{*}\left(T_{1}\right), \ldots, \pi^{*}\left(T_{4}\right)$, as well as a component $W$ strictly containing $\pi^{*}(T)$. As noted in [19], $W$ is a 4-dimensional algebraic subtorus. Direct computation shows that $\mathcal{V}_{1}(F(\mathcal{A}))$ has no other irreducible components.

Example 5.20. - Let $\mathcal{A}$ be the Hessian arrangement from Example 2.15. We know that $\mathcal{V}_{1}(\mathcal{A})$ has 10 components passing through the origin, all 3-dimensional: 9 of those are local components, while the last one, $T$, corresponds to the $(4,3)$-net from Figure 4. As noted in [19], the component $W$ of $\mathcal{V}_{1}(F(\mathcal{A}))$ containing $\pi^{*}(T)$ has dimension 9 . In particular, this shows that $b_{1}(F(\mathcal{A})) \geq 15$.

Problem 5.21. - Find a more precise description of the characteristic varieties $\mathcal{V}_{s}(F(\mathcal{A}))$, and, more generally, $\mathcal{V}_{s}^{1}\left(F_{m}(\mathcal{A}), \mathbb{k}\right)$.

5.6. The formality problem. - The following question was raised in [53], in a more general context: Is the Milnor fiber of a hyperplane arrangement always formal? Of course, if $\operatorname{rank}(\mathcal{A})=2$, then $F(\mathcal{A})$ has the homotopy type of a wedge of circles, and so it is formal. In general, though, the answer is no, as illustrated by the following example from [68].

Example 5.22. - Let $\mathcal{A}$ be the Ceva(3) arrangement from Example 2.14, and let $T$ be the 2-dimensional, essential component of $\mathcal{V}_{1}(\mathcal{A})$ described in Example 5.10. The pullback $\pi^{*}(T)$ is a 4 -dimensional subtorus of $H^{1}\left(F(\mathcal{A}), \mathbb{C}^{*}\right)=\left(\mathbb{C}^{*}\right)^{12}$, of the form $\exp (L)$, for some linear subspace $L \subset H^{1}(F(\mathcal{A}), \mathbb{C})$. Using the mixed Hodge structure on the cohomology of the Milnor fiber, Zuber [68] shows that $L$ cannot possibly be a component of the resonance variety $\mathcal{R}_{1}(F(\mathcal{A})$ ). Hence, the tangent cone formula from Theorem C.4 is violated, and thus, the space $F(\mathcal{A})$ cannot be 1-formal.

This example raises several questions, all interrelated. 
Problem 5.23. - Find a concrete description of the resonance varieties $\mathcal{R}_{s}(F(\mathcal{A})$ ), and, more generally, $\mathcal{R}_{s}^{1}\left(F_{m}(\mathcal{A}), \mathbb{k}\right)$. For which arrangements $\mathcal{A}$ does the tangent cone formula $\operatorname{TC}_{1}\left(\mathcal{V}_{1}(F(\mathcal{A}))\right)=\mathcal{R}_{1}(F(\mathcal{A}))$ hold?

Problem 5.24. - Give a purely topological explanation for the non-formality of the Milnor fibers of arrangements such as the Ceva(3) arrangement.

Such an explanation could involve either an explicit computation of the resonance varieties of Milnor fibers (as in Problem 5.23), or the study of triple Massey products in the cohomology of the Milnor fiber, or perhaps some completely new method.

Problem 5.25. - Given a multi-arrangement $(\mathcal{A}, m)$, determine whether the Milnor fiber $F_{m}(\mathcal{A})$ is formal or not. Does this formality property depend only on the underlying arrangement $\mathcal{A}$, or also on the multiplicity vector $m$ ?

\section{The boundary manifold of an arrangement}

The boundary manifold of a hyperplane arrangement $\mathcal{A}$ in $\mathbb{C}^{d+1}$ is the boundary of a regular neighborhood in $\mathbb{C P}^{d}$ of the union of the projective hyperplanes comprising $\mathbb{P}(\mathcal{A})$. In this section, we survey a number of known results (primarily from [11, 12]) regarding the cohomology ring, fundamental group, jump loci, and formality properties of boundary manifolds of arrangements.

6.1. The boundary manifold. - Let $\mathcal{A}$ be a (central) arrangement of hyperplanes in $\mathbb{C}^{d+1}(d \geq 1)$ with union $V=\bigcup_{H \in \mathcal{A}} H$ and complement $M=\mathbb{C}^{d+1} \backslash V$. Likewise, let $\mathbb{P}(\mathcal{A})=\{\mathbb{P}(H) \mid H \in \mathcal{A}\}$ be the corresponding arrangement in $\mathbb{C P}^{d}$, with union $W=\mathbb{P}(V)$ and complement $U=\mathbb{P}(M)$. A regular neighborhood $\nu(W)$ of the algebraic hypersurface $W \subset \mathbb{C P}^{d}$ may be constructed as follows.

Let $\phi: \mathbb{C P}^{d} \rightarrow \mathbb{R}$ be the smooth function defined by $\phi([z])=|Q(z)|^{2} /\|z\|^{2 n}$, where $Q$ is a defining polynomial for the arrangement, and $n=|\mathcal{A}|$. Then, for sufficiently small $\delta>0$, the preimage $\phi^{-1}([0, \delta])$ is a closed, regular neighborhood of $W$. (Since $Q$ is homogeneous, we may simply take $\delta=1$.) Alternatively, one may triangulate $\mathbb{C P}^{d}$ with $W$ as a subcomplex, and take $\nu(W)$ to be the closed star of $W$ in the second barycentric subdivision.

As shown by Durfee [22], these constructions yield isotopic neighborhoods, independent of the choices made. Evidently, $\nu(W)$ is a compact, orientable, smooth manifold with boundary, of dimension $2 d$; moreover, $\nu(W)$ deform-retracts onto $W$.

The exterior of the projectivized arrangement, denoted $\bar{U}$, is the complement in $\mathbb{C P}^{d}$ of the open regular neighborhood $\stackrel{\circ}{\nu}(W)$. Clearly, $\bar{U}$ is a compact, connected, orientable, smooth $2 d$-manifold with boundary. Moreover, $U$ deform-retracts onto $\bar{U}$, and thus $\bar{U} \simeq U$.

Definition 6.1. - The boundary manifold of a hyperplane arrangement $\mathcal{A}$ in $\mathbb{C}^{d+1}$ is the common boundary

$$
\partial \bar{U}=\partial \nu(W)
$$

of the exterior $\bar{U}$ and the regular neighborhood $\nu(W)$ defined above. 
Note that $\partial \bar{U}$ is a compact, orientable, smooth manifold (without boundary), of dimension $2 d-1$. The inclusion map $j: \partial \bar{U} \rightarrow \bar{U}$ is a $(d-1)$-equivalence, see [15, Proposition 2.31]; in particular, $\pi_{i}(\partial \bar{U}) \cong \pi_{i}(U)$ for $i<d-1$.

If $d=1$, then $\mathbb{P}(\mathcal{A})$ consists of $n$ distinct points in $\mathbb{C P}^{1}$; thus, the boundary manifold $\partial \bar{U}$ consists of $n$ disjoint small circles around those points, and there is not much else to say. Consequently, we will assume from now on that $d \geq 2$, in which case $\partial \bar{U}$ is connected.

Let us illustrate these definitions with a couple of examples, extracted from [11].

Example 6.2. - Let $\mathcal{A}$ be a pencil of $n$ hyperplanes in $\mathbb{C}^{d+1}$, defined by the polynomial $Q=z_{1}^{n}-z_{2}^{n}$. If $n=1$, then $\bar{U}=D^{2 d}$ and $\partial \bar{U}=S^{2 d-1}$. Otherwise, $U$ may be realized as the complement of $n-1$ parallel hyperplanes in $\mathbb{C}^{d}$. In this case,

$$
\bar{U}=\left(D^{2} \backslash\{n \text { disjoint open disks }\}\right) \times D^{2(n-1)},
$$

and thus $\partial \bar{U}$ is diffeomorphic to the connected sum $\sharp^{n-1} S^{1} \times S^{2(d-1)}$.

Example 6.3. - Let $\mathcal{A}$ be a near-pencil of $n$ planes in $\mathbb{C}^{3}$, defined by the polynomial $Q=z_{1}\left(z_{2}^{n-1}-z_{3}^{n-1}\right)$. In this case, $\partial \bar{U}=S^{1} \times \Sigma_{n-2}$, where $\Sigma_{g}=\sharp^{g} S^{1} \times S^{1}$ denotes the orientable surface of genus $g$ (see also Example 7.4).

6.2. Homology groups and cup products. - As shown in [11], the long exact sequence of the pair $(\bar{U}, \partial \bar{U})$ breaks into split, short exact sequences,

$$
0 \longrightarrow H_{q+1}(\bar{U}, \partial \bar{U}, \mathbb{Z}) \longrightarrow H_{q}(\partial \bar{U}, \mathbb{Z}) \stackrel{j_{*}}{\longrightarrow} H_{q}(\bar{U}, \mathbb{Z}) \longrightarrow 0 .
$$

By Lefschetz duality, $H_{q+1}(\bar{U}, \partial \bar{U}, \mathbb{Z}) \cong H^{2 d-q-1}(\bar{U}, \mathbb{Z})$. Since $\bar{U} \simeq U$, and since the homology groups of $U$ are torsion-free, we obtain a direct sum decomposition

$$
H_{q}(\partial \bar{U}, \mathbb{Z}) \cong H_{q}(U, \mathbb{Z}) \oplus H_{2 d-q-1}(U, \mathbb{Z})
$$

Hence, $\operatorname{Poin}(\partial \bar{U}, t)=\operatorname{Poin}(U, t)+t^{2 d-1} \cdot \operatorname{Poin}\left(U, t^{-1}\right)$.

The cohomology groups of $\partial \bar{U}$ admit a decomposition similar to the one from (49). To describe the cup-product structure in $H^{*}(\partial \bar{U}, \mathbb{Z})$, we first need to review some notions. Let $A$ be a graded, finite-dimensional algebra over $\mathbb{Z}$. Assume that $A$ is graded-commutative, of finite type (i.e., each graded piece $A^{q}$ is a finitely generated $\mathbb{Z}$-module), and connected (i.e., $A^{0}=\mathbb{Z}$ ). Then, the $\mathbb{Z}$-dual $\check{A}=\operatorname{Hom}_{\mathbb{Z}}(A, \mathbb{Z})$ is an $A$-bimodule, with left and right multiplication given by $(a \cdot f)(b)=f(b a)$ and $(f \cdot a)(b)=f(a b)$, respectively. Moreover, this bimodule structure is compatible with the gradings: if $a \in A^{q}$ and $f \in \check{A}^{p}$, then both af and $f a$ belong to $\check{A}^{p-q}$.

Theorem 6.4 ([11]). - Let $\mathcal{A}$ be an arrangement in $\mathbb{C}^{d+1}$, let $A=H^{*}(U, \mathbb{Z})$ be the cohomology ring of the projectivized complement, and let $\widehat{A}=H^{*}(\partial \bar{U}, \mathbb{Z})$ be the cohomology ring of the boundary manifold. Then $\widehat{A}=A \oplus \check{A}$, with multiplication given by $(a, f) \cdot(b, g)=(a b, a g+f b)$, and grading $\widehat{A}^{q}=A^{q} \oplus \check{A}^{2 d-q-1}$.

Consequently, the cohomology ring of the boundary manifold depends only on the intersection lattice of the arrangement. In the case when $d=2$, the structure of this ring can be described in more concrete terms, as follows. 
Write $A=A^{0} \oplus A^{1} \oplus A^{2}$, and fix ordered bases, $\left\{\alpha_{1}, \ldots, \alpha_{n}\right\}$ for $A^{1}$ and $\left\{\beta_{1}, \ldots, \beta_{m}\right\}$ for $A^{2}$. The multiplication map, $\mu: A^{1} \otimes A^{1} \rightarrow A^{2}$, is then given by $\mu\left(\alpha_{i}, \alpha_{j}\right)=$ $\sum_{k=1}^{m} \mu_{i j k} \beta_{k}$, for some integer coefficients satisfying $\mu_{j i k}=-\mu_{i j k}$. Now write $\widehat{A}=$ $A^{0} \oplus\left(A^{1} \oplus \check{A}^{2}\right) \oplus\left(A^{2} \oplus \check{A}^{1}\right) \oplus \check{A}^{0}$, and pick dual bases $\left\{\alpha_{1}^{\prime}, \ldots, \alpha_{n}^{\prime}\right\}$ for $\check{A}^{1}$ and $\left\{\beta_{1}^{\prime}, \ldots, \beta_{m}^{\prime}\right\}$ for $\check{A}^{2}$. The multiplication map $\hat{\mu}: \widehat{A}^{1} \otimes \widehat{A}^{1} \rightarrow \widehat{A}^{2}$ restricts to $\mu$ on $A^{1} \otimes A^{1}$, vanishes on $\check{A}^{2} \otimes \check{A}^{2}$, while on $A^{1} \otimes \check{A}^{2}$, it is given by $\hat{\mu}\left(\alpha_{j}, \beta_{k}^{\prime}\right)=\sum_{i=1}^{n} \mu_{i j k} \alpha_{i}^{\prime}$. Finally, $\hat{\mu}\left(\alpha_{i}, \alpha_{j}^{\prime}\right)=\hat{\mu}\left(\beta_{i}, \beta_{j}^{\prime}\right)=\delta_{i j} \omega$, where $\omega$ is the generator of $\tilde{A}^{0}$ dual to $1 \in A^{0}$.

6.3. Resonance varieties. - The resonance varieties of the boundary manifold of an arrangement $\mathcal{A}$ in $\mathbb{C}^{d+1}$ were studied in detail in [11, 12]. If $d \geq 3$, then $H^{1}(\partial \bar{U}, \mathbb{C})=H^{1}(U, \mathbb{C})$, and the resonance varieties of $\partial \bar{U}$ can be expressed solely in terms of the resonance varieties of $U$ :

$$
\mathcal{R}_{s}^{q}(\partial \bar{U})= \begin{cases}\mathcal{R}_{s}^{q}(U) & \text { if } q \leq d-2, \\ \bigcup_{i+j=s}\left(\mathcal{R}_{i}^{d-1}(U) \cap \mathcal{R}_{j}^{d}(U)\right) & \text { if } q=d-1 \text { or } q=d, \\ \mathcal{R}_{s}^{2 d-k-1}(U) & \text { if } q \geq d+1 .\end{cases}
$$

Now suppose $\mathcal{A}$ is an arrangement of $n$ planes in $\mathbb{C}^{3}$, and let us consider the resonance varieties $\mathcal{R}_{s}^{1}(\partial \bar{U})$ of its boundary manifold. For depth $s=1$, these varieties admit a particularly simple description:

$$
\mathcal{R}_{1}^{1}(\partial \bar{U})= \begin{cases}\mathbb{C}^{n-1} & \text { if } \mathcal{A} \text { is a pencil, } \\ \mathbb{C}^{2(n-2)} & \text { if } \mathcal{A} \text { is a near-pencil, } \\ H^{1}(\partial \bar{U}, \mathbb{C}) & \text { otherwise }\end{cases}
$$

The higher-depth resonance varieties, though, can be much more complicated, as the following example from [11, Corollary 6.11] illustrates.

Example 6.5. - Let $\mathcal{A}$ be an arrangement of $n+1$ planes in $\mathbb{C}^{3}$, in general position. If $n \geq 4$ and $\left(\begin{array}{l}n \\ 2\end{array}\right)<s<\left(\begin{array}{l}n \\ 2\end{array}\right)+n-2$, then the resonance variety $\mathcal{R}_{s}^{1}(\partial \bar{U})$ is the zero locus of the Pfaffians (of appropriate size) of a generic $n \times n$ skew-symmetric matrix. In particular, $\mathcal{R}_{s}^{1}(\partial \bar{U})$ is a singular, irreducible variety.

6.4. Graph manifold structure. - As noted in $\S 6.1$, if $\mathcal{A}$ is an arrangement in $\mathbb{C}^{d+1}$, with $d>3$, then $\pi_{1}(\partial \bar{U})=\pi_{1}(U)$. Thus, from the point of view of the fundamental group of the boundary manifold, the most interesting dimension to study is $d=3$. So assume for the rest of this section that $\mathcal{A}$ is an arrangement of planes in $\mathbb{C}^{3}$. In this case, the boundary manifold admits another interpretation, that arises in the work of Jiang and Yau [32,33], Hironaka [30], and Westlund [64].

Let $\mathbb{P}(\mathcal{A})=\left\{\ell_{1}, \ldots, \ell_{n}\right\}$ be the projectivized line arrangement in $\mathbb{C P}^{2}$. Suppose $\mathbb{P}(\mathcal{A})$ has $r$ non-transverse intersection points, i.e., points $p_{J}=\bigcap_{j \in J} \ell_{j}$, of multiplicity $|J| \geq 3$. Blowing up $\mathbb{C P}^{2}$ at each of these points, we obtain an arrangement $\widetilde{\mathcal{A}}=$ $\left\{L_{1}, \ldots, L_{n+r}\right\}$ in the rational surface $\widetilde{\mathbb{C P}}^{2} \cong \mathbb{C P}^{2} \# r \overline{\mathbb{C P}}^{2}$, consisting of the proper transforms of the lines of $\mathbb{P}(\mathcal{A})$, together with the exceptional lines arising from the blow-ups. 
This construction realizes the boundary manifold $\partial \bar{U}$ as a graph manifold, in the sense of Waldhausen. The underlying graph $\Gamma$ has vertex set $\mathrm{V}$, with vertices in oneto-one correspondence with the lines of $\widetilde{\mathcal{A}}$ : the vertex corresponding to $\ell_{i}$ is labeled $v_{i}$, while the vertex corresponding to $p_{J}$ is labeled $v_{J}$. The edge set $\mathrm{E}$ consists of an edge $e_{i, j}$ from $v_{i}$ to $v_{j}$ if $i<j$ and the corresponding lines $\ell_{i}$ and $\ell_{j}$ are transverse, and an edge $e_{J, i}$ from $v_{J}$ to $v_{i}$ if $p_{J} \in \ell_{i}$. Each vertex gets assigned a weight, equal to the self-intersection number of the corresponding line in the blow-up: $v_{i}$ has weight $w_{i}=1-\left|\left\{J \mid p_{J} \in \ell_{i}\right\}\right|$, and $v_{J}$ has weight $w_{J}=-1$.

6.5. Fundamental group. - Applying a method due to Hirzebruch [31] to the graph manifold structure described above, Westlund [64] obtained a presentation for the fundamental group of the boundary manifold of an arrangement in $\mathbb{C}^{3}$, as follows.

Let $\Gamma$ be the weighted graph associated to $\mathbb{P}(\mathcal{A})$, and choose an orientation on this graph. Pick a maximal tree $\mathrm{T} \subset \mathrm{E}$, and list the remaining edges as $e_{1}, \ldots, e_{s}$, where $s$ is the number of linearly independent cycles in $\Gamma$. With these choices, the fundamental group of $\partial \bar{U}(\mathcal{A})$ has presentation

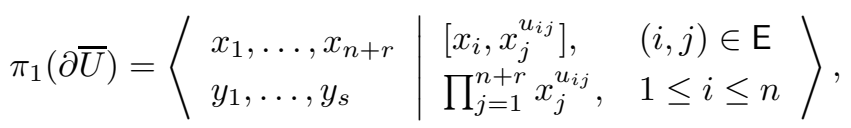

where

$$
u_{i j}= \begin{cases}w_{i} & \text { if } i=j, \\ y_{k} & \text { if }(i, j) \text { is the } k \text {-th element of } \mathrm{E} \backslash \mathrm{T}, \\ y_{k}^{-1} & \text { if }(j, i) \text { is the } k \text {-th element of } \mathrm{E} \backslash \mathrm{T}, \\ 1 & \text { if }(i, j) \text { or }(j, i) \text { belongs to } \mathrm{T}, \\ 0 & \text { otherwise. }\end{cases}
$$

Here $[a, b]=a b a^{-1} b^{-1}, a^{0}=1$ is the identity element, and $a^{b}=b^{-1} a b$ for $b \neq 0$. Note that if $i \neq j$ and $u_{i j} \neq 0$, then $u_{j i}=u_{i j}^{-1}$.

Proposition 6.6 ([12]). - The presentation (52) may be simplified to a commutator-relators presentation for $\pi_{1}(\partial \bar{U})$, with generators $x_{1}, \ldots, x_{n-1}$ and $y_{1}, \ldots, y_{s}$ as above.

Instead of reproducing the precise set of relations here, let us first make a remark, and then illustrate with a simple example.

Remark 6.7. - Recall from (48) that the inclusion map $j: \partial \bar{U} \rightarrow \bar{U}$ induces a split surjection in first homology, leading to the direct sum decomposition $H_{1}(\partial \bar{U}, \mathbb{Z}) \cong$ $H_{1}(\bar{U}, \mathbb{Z}) \oplus H_{2}(\bar{U}, \partial \bar{U}, \mathbb{Z})$. The group $H_{1}(\bar{U}, \mathbb{Z})=H_{1}(U, \mathbb{Z})$ is freely generated by homology classes $x_{1}, \ldots, x_{n-1}$ corresponding to the meridians around the first $n-1$ lines. On the other hand, the group $H_{2}(\bar{U}, \partial \bar{U}, \mathbb{Z})$ is isomorphic to $H_{1}(\Gamma, \mathbb{Z})$, and thus admits a basis $y_{1}, \ldots, y_{s}$ corresponding to disks in the exterior whose boundaries are the chosen cycles in the graph. With respect to these generating sets, then, the abelianization map ab: $\pi_{1}(\partial \bar{U}) \rightarrow H_{1}(\partial \bar{U}, \mathbb{Z})$ takes $x_{i}$ to $x_{i}$ and $y_{i}$ to $y_{i}$, while the induced homomorphism $j_{*}: H_{1}(\partial \bar{U}, \mathbb{Z}) \rightarrow H_{1}(\bar{U}, \mathbb{Z})$ takes $x_{i}$ to $x_{i}$ and $y_{i}$ to 0 . 

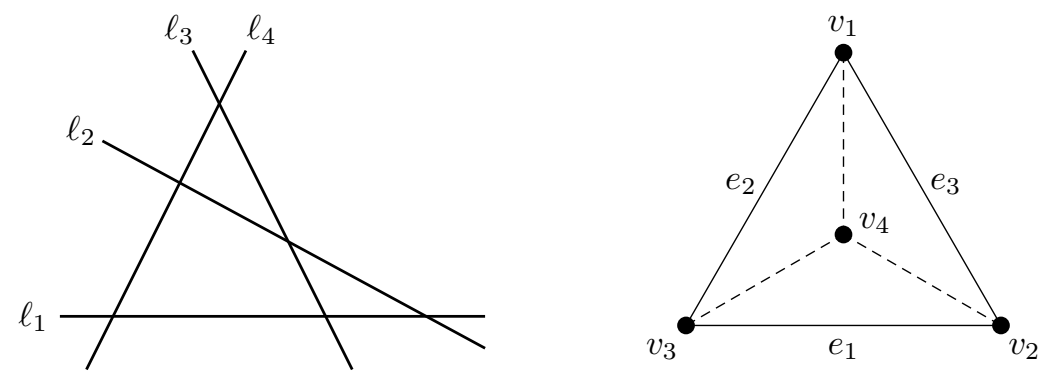

FiguRE 6. A general position arrangement and its associated graph

Example 6.8. - Let $\mathcal{A}$ be an arrangement of 4 planes in $\mathbb{C}^{3}$ in general position. In this case, $\Gamma$ is the complete graph $K_{4}$. Using the maximal tree indicated by dashed edges in Figure 6, we obtain the following presentation for the fundamental group of the boundary manifold of $\mathcal{A}$ :

$$
\pi_{1}(\partial \bar{U})=\left\langle\begin{array}{l|l}
x_{1}, x_{2}, x_{3} & x_{1} x_{2} x_{3}=x_{1} x_{2}^{y_{1}} x_{3}^{y_{2}}=x_{2} x_{1}^{y_{3}^{-1}} x_{3}^{y_{1}}=x_{3} x_{1}^{y_{2}^{-1}} x_{2}^{y_{1}^{-1}} \\
y_{1}, y_{2}, y_{3} & {\left[x_{1}, x_{2}^{y_{3}}\right]=\left[x_{1}, x_{3}^{y_{2}}\right]=\left[x_{2}, x_{3}^{y_{1}}\right]=1}
\end{array}\right\rangle .
$$

6.6. Alexander polynomial and characteristic varieties. - The next result expresses the Alexander polynomial of the boundary manifold in terms of the underlying graph structure.

Theorem $6.9([12])$. - Let $\mathcal{A}$ be an essential arrangement of planes in $\mathbb{C}^{3}$, and let $\Gamma$ be the associated graph. Then the Alexander polynomial of the boundary manifold $\partial \bar{U}$ is given by

$$
\Delta_{\partial \bar{U}}=\prod_{v \in \mathrm{V}(\Gamma)}\left(t_{v}-1\right)^{d_{v}-2},
$$

where $d_{v}$ denotes the degree of the vertex $v$, and $t_{v}=\prod_{i \in v} t_{i}$.

Note that $\Delta_{\partial \bar{U}}(1)=0$. In view of Proposition A.2, we obtain the following decomposition of the first characteristic variety of $\partial \bar{U}$ into irreducible components:

$$
\mathcal{V}_{1}^{1}(\partial \bar{U})=\bigcup_{v \in \mathbf{V}(\Gamma): d_{v} \geq 3}\left\{t_{v}-1=0\right\} .
$$

In particular, every component of $\mathcal{V}_{1}^{1}(\partial \bar{U})$ is a codimension 1 algebraic subtorus of the character torus of $\partial \bar{U}$.

6.7. Formality. - As we saw in Examples 6.2 and 6.3, for simple arrangements such as pencils or near-pencils, the boundary manifold is built out of spheres by successive product and connected sum operations, and thus is formal. On the other hand, consider the following, equally simple, example. 
Example 6.10. - Let $\mathcal{A}$ be an arrangement of 5 planes in $\mathbb{C}^{3}$, in general position. From the discussion in Example 6.5, it follows that $\mathcal{R}_{7}^{1}(\partial \bar{U})$ is isomorphic to $\{z \in$ $\left.\mathbb{C}^{6} \mid z_{1} z_{6}-z_{2} z_{5}+z_{3} z_{4}=0\right\}$, an irreducible quadric with an isolated singular point at 0 . By Theorem C.4, then, the manifold $\partial \bar{U}$ is not 1 -formal.

Alternatively, formula (53) implies that $\mathcal{V}_{s}^{1}(\partial \bar{U}) \subseteq\{1\}$, for all $s \geq 1$. Thus, $\mathrm{TC}_{1}\left(\mathcal{V}_{7}^{1}(\partial \bar{U})\right) \neq \mathcal{R}_{7}^{1}(\partial \bar{U})$, showing again that $\partial \bar{U}$ is not 1 -formal.

The general situation was elucidated in [12, Theorem 9.7].

Theorem $6.11([12])$. - Let $\mathcal{A}$ be an arrangement of planes in $\mathbb{C}^{3}$, and let $\partial \bar{U}$ be the corresponding boundary manifold. The following are equivalent:

1. The manifold $\partial \bar{U}$ is formal.

2. The group $\pi_{1}(\partial \bar{U})$ is 1-formal.

3. $\mathrm{TC}_{1}\left(\mathcal{V}_{1}^{1}(\partial \bar{U})\right)=\mathcal{R}_{1}^{1}(\partial \bar{U})$.

4. $\mathcal{A}$ is either a pencil or a near-pencil.

5. $\partial \bar{U}$ is either $\sharp^{n} S^{1} \times S^{2}$ or $S^{1} \times \Sigma_{n-1}$, where $n=|\mathcal{A}|-1$.

Proof. - Implications (1) $\Rightarrow(2) \Rightarrow(3)$ are true in general (see Appendix C), implication $(4) \Rightarrow(5)$ was discussed in Examples 6.2 and 6.3 , while $(5) \Rightarrow(1)$ is obvious.

To prove (3) $\Rightarrow(4)$, suppose $\mathcal{A}$ is neither a pencil, nor a near-pencil. Then, by formula (51), $\mathcal{R}_{1}^{1}(\partial \bar{U})=H^{1}(\partial \bar{U}, \mathbb{C})$. On the other hand, formula (53) shows that $\mathcal{V}_{1}^{1}(\partial \bar{U})$ is a union of codimension 1 subtori in $H^{1}\left(\partial \bar{U}, \mathbb{C}^{*}\right)$. Thus, $\operatorname{TC}_{1}\left(\mathcal{V}_{1}^{1}(\partial \bar{U})\right) \neq$ $\mathcal{R}_{1}^{1}(\partial \bar{U})$, and we are done.

\section{The boundary of the Milnor fiber of an arrangement}

In this section, we study the boundary of the closed Milnor fiber of a hyperplane arrangement, and the way it relates to the boundary manifold of the arrangement.

7.1. The closed Milnor fiber. - As usual, let $\mathcal{A}$ be a (central) arrangement of hyperplanes in $\mathbb{C}^{d+1}(d \geq 1)$, with defining polynomial $Q(\mathcal{A})=\prod_{H \in \mathcal{A}} f_{H}$. Let $V=$ $V(\mathcal{A})$ be the union of the hyperplanes in $\mathcal{A}$, and let $M=M(\mathcal{A})$ be its complement.

Now let $m$ be a (primitive) multiplicity vector for $\mathcal{A}$, and let $Q_{m}(\mathcal{A})=\prod_{H \in \mathcal{A}} f_{H}^{m_{H}}$. As mentioned in $\S 4$, the polynomial map $Q_{m}=Q_{m}(\mathcal{A}): \mathbb{C}^{d+1} \rightarrow \mathbb{C}$ restricts to a smooth fibration, $Q_{m}: M \rightarrow \mathbb{C}^{*}$, with fiber $F=F_{m}(\mathcal{A})$.

Intersecting the global Milnor fiber with a ball in $\mathbb{C}^{d+1}$ of large enough radius, we obtain a compact, smooth, orientable $2 d$-dimensional manifold with boundary,

$$
\bar{F}_{m}(\mathcal{A})=F_{m}(\mathcal{A}) \cap D^{2(d+1)},
$$

which we call the closed Milnor fiber of the multi-arrangement. Clearly, $F_{m}(\mathcal{A})$ deform-retracts onto $\bar{F}_{m}(\mathcal{A})$; hence, $\bar{F}_{m}(\mathcal{A}) \simeq F_{m}(\mathcal{A})$.

The boundary of the Milnor fiber of $(\mathcal{A}, m)$ is the compact, smooth, orientable, $(2 d-1)$-dimensional manifold

$$
\partial \bar{F}_{m}(\mathcal{A})=F_{m}(\mathcal{A}) \cap S^{2 d+1} .
$$



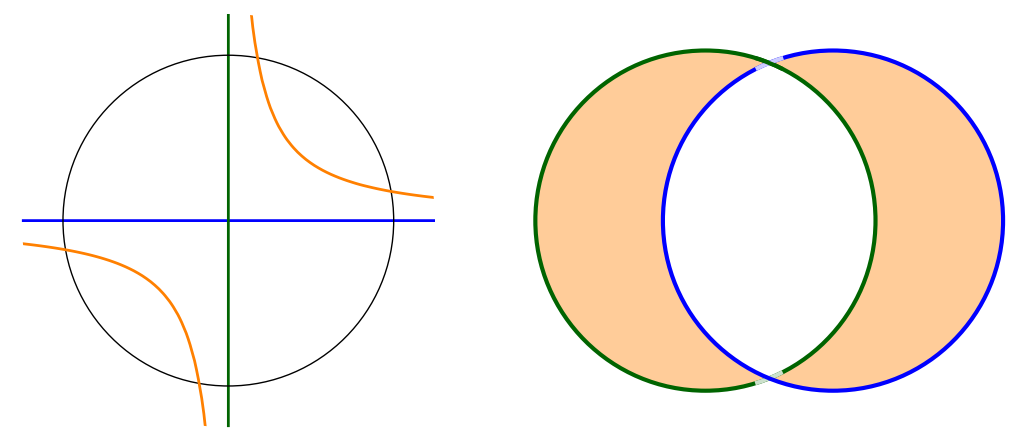

FiguRE 7. Local Milnor fibration and closed Milnor fiber for $Q(\mathcal{A})=x y$

As usual, we will abbreviate $\bar{F}(\mathcal{A})$ and $\partial \bar{F}(\mathcal{A})$ when all multiplicities $m_{H}$ are equal to 1 , and will drop $\mathcal{A}$ from the notation when the arrangement is understood. As noted in [15, Proposition 2.4], the pair $\left(\bar{F}_{m}, \partial \bar{F}_{m}\right)$ is $(d-1)$-connected. In particular, if $d \geq 2$, the boundary of the Milnor fiber is connected, and the inclusion-induced homomorphism $\pi_{1}\left(\partial \bar{F}_{m}\right) \rightarrow \pi_{1}\left(\bar{F}_{m}\right)$ is surjective.

7.2. The local Milnor fibration. - Returning to the general situation, let $F_{m}=$ $F_{m}(\mathcal{A})$ be the global Milnor fiber of a multi-arrangement. Recall that the monodromy of the fibration $F_{m} \rightarrow M \rightarrow \mathbb{C}^{*}$ is the diffeomorphism $h: F_{m} \rightarrow F_{m}$ given by $h(z)=$ $e^{2 \pi \mathrm{i} / N} z$, where $N=\sum_{H \in \mathcal{A}} m_{H}$.

Clearly, the map $h$ restricts to a diffeomorphism $h: \bar{F}_{m} \rightarrow \bar{F}_{m}$. Let $Y_{m}(\mathcal{A})$ be the mapping torus of this diffeomorphism; we then have a smooth fibration

$$
\bar{F}_{m} \longrightarrow Y_{m}(\mathcal{A}) \longrightarrow S^{1} .
$$

Now let $K=V \cap S^{2 d+1}$ be the link of the singularity, and let $\nu(K)$ be a closed, regular neighborhood of $K$ inside the sphere. Then, as shown by Milnor in [45], there is a smooth fibration

$$
\bar{F}_{m} \longrightarrow S^{2 d+1} \backslash \stackrel{\circ}{\nu}(K) \stackrel{Q_{m} /\left|Q_{m}\right|}{\longrightarrow} S^{1} .
$$

Furthermore, the local Milnor fibration (57) is equivalent, through a fiber-preserving diffeomorphism, to the fibration (56).

Example 7.1. - Let $\mathcal{A}$ be the arrangement in $\mathbb{C}^{2}$ defined by the polynomial $Q(\mathcal{A})=$ $z_{1}^{n}-z_{2}^{n}$. Then $\bar{F}(\mathcal{A})$ is a Seifert surface for the $n$-component Hopf link. This is a compact, connected, orientable surface of genus $\left(\begin{array}{c}n-1 \\ 2\end{array}\right)$, with $n$ open disks removed; its boundary, $\partial \bar{F}(\mathcal{A})$, consists of $n$ disjoint circles.

For $n=2$, the surface is the twisted cylinder depicted in Figure 7, and the monodromy is a Dehn twist about the core of the cylinder. (Although this diffeomorphism is isotopic to the identity, no such isotopy can be the identity on the two boundary circles.) 
7.3. The double of the Milnor fiber. - There are several ways in which the closed Milnor fiber and its boundary are related to the boundary manifold of an arrangement. The next proposition (whose proof is adapted from the discussion in $[11, \S 2.8]$ ) details one such relationship, in the special case when the defining polynomial splits off a linear factor not involving the other variables.

First recall a basic notion: the double of a manifold $W$ with non-empty boundary $\partial W$ is the (closed) manifold $\partial(W \times[0,1])=W \cup_{\partial W} W$.

Proposition 7.2. - Let $\mathcal{A}$ be a hyperplane arrangement in $\mathbb{C}^{d+1}$, defined by a polynomial of the form $Q\left(z_{0}, \ldots, z_{d}\right)=z_{0} Q_{0}\left(z_{1}, \ldots, z_{d}\right)$. Let $\mathcal{A}_{0}$ be the arrangement in $\mathbb{C}^{d}$ defined by $Q_{0}$, and let $\bar{F}_{0} \rightarrow Y_{0} \rightarrow S^{1}$ be the corresponding Milnor fibration. Then, the boundary manifold of $\mathcal{A}$ is diffeomorphic to the double of $Y_{0} ;$ moreover, $\partial \bar{U}(\mathcal{A})$ fibers over the circle, with fiber the double of $\bar{F}_{0}$.

Proof. - Let $V_{0}$ be the subvariety of $\mathbb{C}^{d}$ defined by $Q_{0}$. Its projective closure, $\bar{V}_{0}$, is the zero-set of $Q$; moreover, $\mathbb{C P}^{d} \backslash \bar{V}_{0}=\mathbb{C}^{d} \backslash V_{0}$. Forming the union of a regular neighborhood $\nu\left(V_{0}\right)$ with a tubular neighborhood of the hyperplane at infinity, , and rounding off corners, we obtain a regular neighborhood $\nu\left(\bar{V}_{0}\right)$. Clearly, $\mathbb{C P}^{d} \backslash \stackrel{\circ}{\nu}\left(\bar{V}_{0}\right)$ is diffeomorphic to $D^{2 d} \backslash\left(D^{2 d} \cap \stackrel{\circ}{\nu}\left(V_{0}\right)\right)$. Hence,

$$
\partial \bar{U}=\left(S^{2 d-1} \backslash\left(S^{2 d-1} \cap \nu\left(V_{0}\right)\right)\right) \cup\left(D^{2 d} \cap \partial \nu\left(V_{0}\right)\right) .
$$

By the discussion from $\S 7.2$, each of the two sides in the above decomposition is diffeomorphic to $Y_{0}$, and the gluing is done along their common boundary, $\partial Y_{0}$. Thus, $\partial \bar{U}$ is the double of $Y_{0}$. The last assertion follows at once.

Example 7.3. - Let $\mathcal{B}_{n}$ be the Boolean arrangement in $\mathbb{C}^{n}$. From Example 4.3, we know that $F\left(\mathcal{B}_{n}\right)=\left(\mathbb{C}^{*}\right)^{n-1}$. Hence, $\bar{F}=\left(S^{1} \times[0,1]\right)^{n-1} \cong T^{n-1} \times D^{n-1}$, and so $\partial \bar{F}=T^{n-1} \times S^{n-2}($ compare with [15, $\S 3$, Exercise 1.15]).

Now note that $Q\left(\mathcal{B}_{n}\right)=z_{0} Q\left(\mathcal{B}_{n-1}\right)$. We also know from Example 4.3 that the bundle $\bar{F}\left(\mathcal{B}_{n-1}\right) \rightarrow Y\left(\mathcal{B}_{n-1}\right) \rightarrow S^{1}$ is trivial. Applying Proposition 7.2, then, shows that $\partial \bar{U}=T^{n-1} \times S^{n-2}$.

Example 7.4. - Let $\mathcal{A}$ be a near-pencil of $n$ planes in $\mathbb{C}^{3}$, defined by the polynomial $Q=z_{0} Q_{0}$, where $Q_{0}=z_{1}^{n-1}-z_{2}^{n-1}$. Then $Y_{0}$ admits a fibration over the circle (different from the Milnor fibration), whose fiber is $D^{2}$ with $n-1$ open disks removed, and whose monodromy is a Dehn twist about the boundary of $D^{2}$. Hence, $\partial \bar{U}=$ $S^{1} \times \Sigma_{n-2}$. As noted in [46, Example 19.10.7], we also have that $\partial \bar{F}=S^{1} \times \Sigma_{n-2}$.

7.4. The boundary of the Milnor fiber as a cyclic cover. - We now describe a construction that realizes the boundary of the Milnor fiber associated to a hyperplane arrangement $\mathcal{A}$ as a regular, finite cyclic cover of the boundary manifold of the arrangement $\mathcal{A}$. As usual, set $n=|\mathcal{A}|$.

Recall that the Hopf fibration $\pi: \mathbb{C}^{d+1} \backslash\{0\} \rightarrow \mathbb{C P}^{d}$ restricts to a (trivializable) bundle map, $\pi: M \rightarrow U$, with fiber $\mathbb{C}^{*}$, which in turn restricts to a regular $\mathbb{Z}_{n}$-cover, $\pi: F \rightarrow U$. 
Lemma 7.5. - The map $\pi: \mathbb{C}^{d+1} \backslash\{0\} \rightarrow \mathbb{C P}^{d}$ restricts to regular, cyclic $n$-fold covers, $\pi: \bar{F} \rightarrow \bar{U}$ and $\pi: \partial \bar{F} \rightarrow \partial \bar{U}$.

Proof. - Let $Q=Q(\mathcal{A})$. Recall that

$$
\begin{aligned}
& \bar{F}=\left\{z \in \mathbb{C}^{d+1} \mid\|z\| \leq 1 \text { and } Q(z)=1\right\}, \text { and } \\
& \bar{U}=\left\{\left.[z] \in \mathbb{C P}^{2 d}|| Q(z)\right|^{2} /\|z\|^{2 n} \geq 1\right\} .
\end{aligned}
$$

Thus, the map $\pi$ restricts to a map of pairs, $\pi:(\bar{F}, \partial \bar{F}) \rightarrow(\bar{U}, \partial \bar{U})$. Hence, the cover $\pi: F \rightarrow U$ restricts to covers $\pi: \bar{F} \rightarrow \bar{U}$ and $\pi: \partial \bar{F} \rightarrow \partial \bar{U}$, and we are done.

Note that the inclusion $\bar{F} \rightarrow F$ is a fiber-preserving homotopy equivalence. Summarizing, we have a commuting ladder

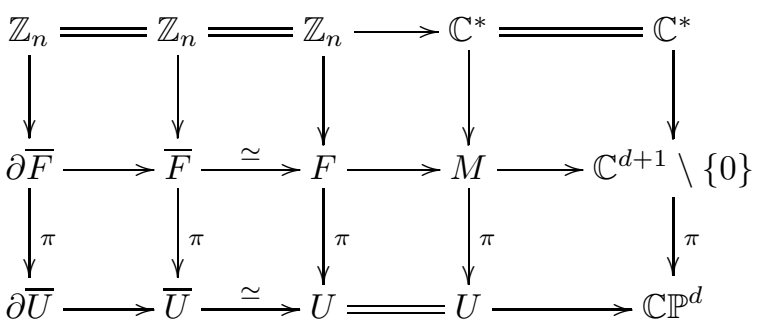

where the horizontal arrows are inclusions, and the maps denoted by $\pi$ are principal bundles with fiber either $\mathbb{C}^{*}$ or $\mathbb{Z}_{n}$.

Assume now that $d=2$, and let $\mathbb{P}(\mathcal{A})=\left\{\ell_{1}, \ldots, \ell_{n}\right\}$ be the associated arrangement of lines in $\mathbb{C P}^{2}$. By Proposition 6.6 , the group $\pi_{1}(\partial \bar{U})$ has generators $x_{1}, \ldots, x_{n-1}$ corresponding to the meridians around the first $n-1$ lines, and generators $y_{1}, \ldots, y_{s}$ corresponding to the cycles in the associated graph $\Gamma$.

Proposition 7.6. - The $\mathbb{Z}_{n}$-cover $\pi: \partial \bar{F} \rightarrow \partial \bar{U}$ is classified by the homomorphism $\pi_{1}(\partial \bar{U}) \rightarrow \mathbb{Z}_{n}$ given by $x_{i} \mapsto 1$ and $y_{i} \mapsto 0$.

Proof. - By Lemma 7.5, the cover $\pi: \partial \bar{F} \rightarrow \partial \bar{U}$ is the pullback along the inclusion $j: \partial \bar{U} \rightarrow \bar{U}$ of the cover $\pi: \bar{F} \rightarrow \bar{U}$. By Theorem 4.10, this latter cover is classified by the homomorphism $\pi_{1}(\bar{U}) \rightarrow \mathbb{Z}_{n}, x_{i} \mapsto 1$. By Remark 6.7, the homomorphism $j_{*}: H_{1}(\partial \bar{U}, \mathbb{Z}) \rightarrow H_{1}(\bar{U}, \mathbb{Z})$ is given by $x_{i} \mapsto x_{i}$ and $y_{i} \mapsto 0$. The desired conclusion follows.

Example 7.7. - Let $\mathcal{A}$ be a pencil of $n+1$ planes in $\mathbb{C}^{3}$. From Example 6.2, we know that $\partial \bar{U}=\sharp^{n} S^{1} \times S^{2}$. Since $\partial \bar{F} \rightarrow \partial \bar{U}$ is a cover with $n+1$ sheets, we find that $\pi_{1}(\partial \bar{F})=F_{n^{2}}$. Hence, by standard 3-manifold topology, $\partial \bar{F}=\sharp^{2} S^{1} \times S^{2}$. (See also [46, Example 19.10.6].)

7.5. The characteristic polynomial of the monodromy. - A detailed study of the boundary of the Milnor fiber of a non-isolated surface singularity was done by Némethi and Szilárd in [46]. When applied to arrangements in $\mathbb{C}^{3}$, their work yields the following result. 
Theorem $7.8([46])$. - Let $\mathcal{A}$ be an arrangement of $n$ planes in $\mathbb{C}^{3}$. The characteristic polynomial of the algebraic monodromy acting on $H_{1}(\partial \bar{F}, \mathbb{C})$ is given by

$$
\Delta(t)=\prod_{X \in L_{2}(\mathcal{A})}(t-1)\left(t^{\operatorname{gcd}(\mu(X)+1, n)}-1\right)^{\mu(X)-1} .
$$

In particular, the first Betti number of $\partial \bar{F}$ is determined solely by the Möbius function of $L(\mathcal{A})$ :

$$
b_{1}(\partial \bar{F})=\sum_{X \in L_{2}(\mathcal{A})}(1+(\mu(X)-1) \operatorname{gcd}(\mu(X)+1, n)) .
$$

This shows that the first Betti number of the boundary of the Milnor fiber is a much less subtle invariant than $b_{1}(F)$, in that $b_{1}(\partial \bar{F})$ depends only on the number and type of multiple points of $\mathbb{P}(\mathcal{A})$, but not on their relative position. The next example illustrates this phenomenon.

Example 7.9. - Let $\mathcal{A}_{1}$ and $\mathcal{A}_{2}$ be the two arrangements from Example 5.11. Recall that the characteristic polynomials of the monodromy operators acting on the first homology of the Milnor fibers of these arrangements are $\Delta_{1}(t)=(t-1)^{8}\left(t^{2}+t+1\right)$ and $\Delta_{2}(t)=(t-1)^{8}$, respectively. On the other hand, the monodromy operators acting on the first homology of the boundaries of the respective Milnor fibers share the same characteristic polynomial, namely, $\Delta(t)=(t-1)^{27}\left(t^{2}+t+1\right)^{9}$.

Nevertheless, there are pairs of arrangements for which the Milnor fibers have the same first Betti number, but the boundaries of the Milnor fibers have different first Betti numbers. Let us illustrate this claim with a simple example.

Example 7.10. - Let $\mathcal{A}_{1}$ be an arrangement of 4 planes in general position and let $\mathcal{A}_{2}$ be a near-pencil of 4 planes. Let $\bar{F}_{1}$ and $\bar{F}_{2}$ be the respective closed Milnor fibers. The monodromy operators acting on $H_{1}\left(\bar{F}_{1}, \mathbb{C}\right)$ and $H_{1}\left(\bar{F}_{2}, \mathbb{C}\right)$ have characteristic polynomial $\Delta(t)=(t-1)^{3}$, yet the monodromy operators acting on $H_{1}\left(\partial \bar{F}_{1}, \mathbb{C}\right)$ and $H_{1}\left(\partial \bar{F}_{2}, \mathbb{C}\right)$ have characteristic polynomials equal to $\Delta_{1}(t)=(t-1)^{6}$ and $\Delta_{2}(t)=$ $(t-1)^{5}$, respectively.

7.6. The formality question. - Next, we determine which Milnor fibers of plane arrangements have formal boundaries.

Proposition 7.11. - Let $\mathcal{A}$ be an arrangement of planes in $\mathbb{C}^{3}$, and let $\partial \bar{F}$ be the boundary of its Milnor fiber. The following are equivalent:

1. The manifold $\partial \bar{F}$ is formal.

2. $\mathcal{A}$ is either a pencil or a near-pencil.

3. $\partial \bar{F}$ is either $\sharp^{2} S^{1} \times S^{2}$ or $S^{1} \times \Sigma_{n-1}$, where $n=|\mathcal{A}|-1$.

Proof. - Implication (2) $\Rightarrow(3)$ was discussed in Examples 7.7 and 7.4, while implication $(3) \Rightarrow(1)$ is obvious.

To prove $(1) \Rightarrow(2)$, suppose $\mathcal{A}$ is neither a pencil, nor a near-pencil. By Theorem 6.11 , then, the boundary manifold $\partial \bar{U}$ is not formal. On the other hand, we know 
from Lemma 7.5 that $\partial \bar{F}$ is a regular, cyclic $n$-fold cover of $\partial \bar{U}$. Hence, by Lemma C.1, the manifold $\partial \bar{F}$ is not formal.

7.7. Further considerations. - In [46, §24], Némethi and A. Szilárd list a number of open problems regarding the boundary of the Milnor fiber of a non-isolated surface singularity. Problem 24.4.19 on the list reads as follows.

Problem 7.12. - Find a nice formula for the torsion of $H_{1}(\partial \bar{F}, \mathbb{Z})$.

The next example shows that torsion can indeed occur in the first homology of $\partial \bar{F}$ (compare with [46, Example 19.10.9]).

Example 7.13. - Let $\mathcal{A}$ be a general position arrangement of 4 planes in $\mathbb{C}^{3}$. As noted above, the characteristic polynomial of the algebraic monodromy acting on $H_{1}(\partial \bar{F}, \mathbb{C})$ is given by $\Delta(t)=(t-1)^{6}$. Direct computation shows that, in fact, $H_{1}(\partial \bar{F}, \mathbb{Z})=\mathbb{Z}^{6} \oplus \mathbb{Z}_{4}$.

For a generic arrangement of $n$ planes in $\mathbb{C}^{3}$, we expect that

$$
H_{1}(\partial \bar{F}, \mathbb{Z})=\mathbb{Z}^{n(n-1) / 2} \oplus \mathbb{Z}_{n}^{(n-2)(n-3) / 2} .
$$

For an arbitrary arrangement $\mathcal{A}$, it would be interesting to see whether all the torsion in $H_{1}(\partial \bar{F}(\mathcal{A}), \mathbb{Z})$ consists of $\mathbb{Z}_{n}$-summands, where $n=|\mathcal{A}|$.

The next problem summarizes Problems 24.2.1 and 24.2.2 from [46].

Problem 7.14. - Determine the cohomology ring and the resonance varieties of the boundary of the Milnor fiber of an arrangement.

Let $\mathcal{A}$ be an arrangement. From Lemma 7.5, we know that the Hopf fibration restricts to a a regular, finite cover, $\pi: \partial \bar{F} \rightarrow \partial \bar{U}$. Proposition B.3, then, insures that $\pi^{*}\left(\mathcal{V}_{s}^{q}(\partial \bar{U}, \mathbb{k})\right) \subseteq \mathcal{V}_{s}^{q}(\partial \bar{F}, \mathbb{k})$ and $\pi^{*}\left(\mathcal{R}_{s}^{q}(\partial \bar{U}, \mathbb{k})\right) \subseteq \mathcal{R}_{s}^{q}(\partial \bar{F}, \mathbb{k})$, for all $q \geq 0$ and $s \geq 1$.

If $\mathcal{A}$ is a near-pencil of planes in $\mathbb{C}^{3}$, then all these inclusions are, in fact, equalities. In general, though, these inclusions are strict. For instance, if $\mathcal{A}$ is a pencil of $n+1$ planes in $\mathbb{C}^{3}$, and $n \geq 2$, then $\mathcal{R}_{1}^{1}(\partial \bar{U}, \mathbb{k})=H^{1}(\partial \bar{U}, \mathbb{k})=\mathbb{k}^{n}$, whereas $\mathcal{R}_{1}^{1}(\partial \bar{F}, \mathbb{k})=$ $H^{1}(\partial \bar{F}, \mathbb{k})=\mathbb{k}^{n^{2}}$.

A

\section{Cohomology jumping loci}

A.1. Characteristic varieties. - Let $X$ be a connected CW-complex. Without loss of generality, we may assume $X$ has a single 0-cell. Let $G=\pi_{1}\left(X, x_{0}\right)$ be the fundamental group of $X$, based at this 0 -cell. We will assume throughout that $X$ has finitely many 1-cells. Of course, these cells must be attached at the unique 0-cell $x_{0}$. By cellular approximation, the based homotopy classes of the 1-cells generate the fundamental group; hence, $G$ is a finitely generated group. 
Now let $\mathbb{k}$ be an algebraically closed field, and let $\widehat{G}=\operatorname{Hom}\left(G, \mathbb{k}^{*}\right)=H^{1}\left(X, \mathbb{k}^{*}\right)$ be the affine algebraic group of $\mathbb{k}$-valued, multiplicative characters on $G$. The characteristic varieties of $X$ over $\mathbb{k}$ are the jumping loci for homology with coefficients in $\mathbb{k}$-valued, rank-1 local systems on $X$ :

$$
\mathcal{V}_{s}^{q}(X, \mathbb{k})=\left\{\rho \in \operatorname{Hom}\left(G, \mathbb{k}^{*}\right) \mid \operatorname{dim}_{\mathbb{k}} H_{q}\left(X, \mathbb{k}_{\rho}\right) \geq s\right\} .
$$

As long as $X$ has finite $q$-skeleton, these loci are Zariski closed subsets of $\widehat{G}$; moreover, we have a descending filtration

$$
\widehat{G}=\mathcal{V}_{0}^{q}(X, \mathbb{k}) \supseteq \mathcal{V}_{1}^{q}(X, \mathbb{k}) \supseteq \cdots \supseteq \mathcal{V}_{s}^{q}(X, \mathbb{k}) \supseteq \cdots .
$$

As shown in [54], the depth-1 characteristic varieties $\mathcal{V}^{q}(X, \mathbb{k})=\mathcal{V}_{1}^{q}(X, \mathbb{k})$ behave well under direct products. More precisely, suppose $X_{1}$ and $X_{2}$ are two connected, finite-type $\mathrm{CW}$-complexes, with fundamental groups $G_{1}$ and $G_{2}$. Identify the character group of $\pi_{1}\left(X_{1} \times X_{2}\right)$ with $\widehat{G_{1}} \times \widehat{G_{2}}$. Then, for all $q \geq 0$,

$$
\mathcal{V}^{q}\left(X_{1} \times X_{2}, \mathbb{k}\right)=\bigcup_{i=0}^{q} \mathcal{V}^{i}\left(X_{1}, \mathbb{k}\right) \times \mathcal{V}^{q-i}\left(X_{2}, \mathbb{k}\right)
$$

A.2. Resonance varieties. - Let $A=H^{*}(X, \mathbb{k})$ be the cohomology algebra of $X$. If $H_{1}(X, \mathbb{Z})$ has 2 -torsion, assume that $\operatorname{char}(\mathbb{k}) \neq 2$. Then, for each $a \in A^{1}$, we have $a^{2}=0$, by graded-commutativity of the cup product. Thus, left-multiplication by $a$ defines a cochain complex,

$$
(A, \cdot a): A^{0} \stackrel{a}{\longrightarrow} A^{1} \stackrel{a}{\longrightarrow} A^{2} \longrightarrow \cdots,
$$

The jump loci for the cohomology of this complex define a natural filtration of the affine space $A^{1}=H^{1}(X, \mathbb{k})$. The resonance varieties of $X$ are the sets

$$
\mathcal{R}_{s}^{q}(X, \mathbb{k})=\left\{a \in A^{1} \mid \operatorname{dim}_{\mathbb{k}} H^{q}(A, a) \geq s\right\} .
$$

If $X$ has finite $q$-skeleton, the sets $\mathcal{R}_{s}^{q}(X, \mathbb{k})$ form a descending filtration of $A^{1}$ by homogeneous, Zariski closed subsets of $A^{1}$. Note that, if $A^{q}=0$, then $\mathcal{R}_{s}^{q}(X, \mathbb{k})=\emptyset$, for all $s>0$. In degree 0 , we have $\mathcal{R}_{1}^{0}(X, \mathbb{k})=\{0\}$, and $\mathcal{R}_{s}^{0}(X, \mathbb{k})=\emptyset$, for $s>1$.

The resonance varieties respect field extensions: if $\mathbb{k} \subseteq \mathbb{K}$, then $\mathcal{R}_{s}^{q}(X, \mathbb{k})=$ $\mathcal{R}_{s}^{q}(X, \mathbb{K}) \cap H^{1}(X, \mathbb{k})$. Furthermore, as noted in [54], the depth-1 resonance varieties $\mathcal{R}^{q}(X, \mathbb{k})=\mathcal{R}_{1}^{q}(X, \mathbb{k})$ behave well under direct products:

$$
\mathcal{R}^{q}\left(X_{1} \times X_{2}, \mathbb{k}\right)=\bigcup_{i=0}^{q} \mathcal{R}^{i}\left(X_{1}, \mathbb{k}\right) \times \mathcal{R}^{q-i}\left(X_{2}, \mathbb{k}\right) .
$$

A.3. Jump loci in degree 1. - Given a finitely generated group $G$, we may define its degree 1 characteristic and resonance varieties as those of a presentation 2-complex for the group. It is readily checked that this definition does not depend on a choice of presentation for $G$.

If the group admits a finite presentation, $G=\left\langle x_{1}, \ldots, x_{n} \mid r_{1}, \ldots, r_{m}\right\rangle$, we may compute the sets $\mathcal{V}_{s}^{1}(G, \mathbb{k})$ and $\mathcal{R}_{s}^{1}(G, \mathbb{k})$ directly from the presentation, by means of the Fox calculus. The algorithm goes as follows. 
Let $F_{n}$ be the free group with generators $x_{1}, \ldots, x_{n}$, and let $\epsilon: \mathbb{k} F_{n} \rightarrow \mathbb{k}$ be the augmentation map, given by $\epsilon\left(x_{i}\right)=1$. For each $1 \leq j \leq n$, there is a linear operator $\partial_{j}=\partial / \partial x_{j}: \mathbb{k} F_{n} \rightarrow \mathbb{k} F_{n}$, known as the $j$-th Fox derivative, uniquely defined by the following rules: $\partial_{j}(1)=0, \partial_{j}\left(x_{i}\right)=\delta_{i j}$, and $\partial_{j}(u v)=\partial_{j}(u) \epsilon(v)+u \partial_{j}(v)$.

Now let $\alpha: \mathbb{k} F_{n} \rightarrow \mathbb{k} G_{\mathrm{ab}}$ be the ring morphism obtained by composing the abelianization map ab: $G \rightarrow G_{\text {ab }}$ with the presentation homomorphism $\phi: F_{n} \rightarrow G$, and extending linearly to group rings. We then define the Alexander matrix of the given presentation as the $m$ by $n$ matrix $\Phi=\Phi_{G}$ with entries

$$
\Phi_{i j}=\alpha\left(\partial_{j} r_{i}\right)
$$

in the group algebra $\mathbb{k} G_{\mathrm{ab}}$. Note that $\mathbb{k} G_{\mathrm{ab}}$ is the coordinate ring of the algebraic group $\widehat{G}=\operatorname{Hom}\left(G, \mathbb{k}^{*}\right)$. The variety $\mathcal{V}_{s}^{1}(G, \mathbb{k})$, then, is the zero locus of the codimension $s$ minors of $\Phi$, at least away from the trivial character, see [29, 54].

A somewhat similar interpretation of the resonance varieties $\mathcal{R}_{s}^{1}(G, \mathbb{k})$ was given in [43], at least in the case when $G$ admits a commutator-relators presentation. More precisely, if all the relators $r_{i}$ belong to $\left[F_{n}, F_{n}\right]$, we may define the linearized Alexander matrix, $\Phi^{\text {lin }}=\Phi_{G}^{\text {lin }}$, as the $m$ by $n$ matrix with entries

$$
\Phi_{i j}^{\operatorname{lin}}=\sum_{k=1}^{n} \epsilon\left(\partial_{k} \partial_{j} r_{i}\right) y_{k}
$$

in the polynomial ring $S=\mathbb{k}\left[y_{1}, \ldots, y_{n}\right]$. Of course, $S$ may be viewed as the coordinate ring of the affine space $H^{1}(G, \mathbb{k})=\mathbb{k}^{n}$. The variety $\mathcal{R}_{s}^{1}(G, \mathbb{k})$, then, is the zero locus of the codimension $s$ minors of $\Phi^{\text {lin }}$. For instance, if $n>1$, then $\mathcal{R}_{s}^{1}\left(F_{n}, \mathbb{k}\right)=\mathbb{k}^{n}$, for all $s<n$.

A.4. A naturality property. - Every group homomorphism $\varphi: G \rightarrow Q$ induces a morphism between character groups, $\hat{\varphi}: \widehat{Q} \rightarrow \widehat{G}$, given by $\hat{\varphi}(\rho)(g)=\varphi(\rho(g))$. Likewise, $\varphi$ induces a homomorphism in cohomology, $\varphi^{*}: H^{*}(Q, \mathbb{k}) \rightarrow H^{*}(G, \mathbb{Q})$. Clearly, if $\varphi$ is surjective, then both $\hat{\varphi}$ and $\varphi^{1}$ are injective.

The next proposition describes a nice functoriality property enjoyed by the characteristic and resonance varieties of groups. The proposition extends results from $[52,62]$; the first part of the proof is modeled on the proof of [62, Lemma 2.13], while the second part of the proof is modeled on the proof of [52, Lemma 5.1].

Proposition A.1. - Let $G$ be a finitely generated group, and let $\varphi: G \rightarrow Q$ be a surjective homomorphism. Then, for each ground field $\mathbb{k}$, and each $s \geq 1$, the following hold.

1. The induced morphism between character groups, $\hat{\varphi}: \widehat{Q} \hookrightarrow \widehat{G}$, restricts to an embedding $\mathcal{V}_{s}^{1}(Q, \mathbb{k}) \hookrightarrow \mathcal{V}_{s}^{1}(G, \mathbb{k})$.

2. The induced morphism between cohomology groups, $\varphi^{*}: H^{1}(Q, \mathbb{k}) \hookrightarrow H^{1}(G, \mathbb{k})$, restricts to an embedding $\mathcal{R}_{s}^{1}(Q, \mathbb{k}) \hookrightarrow \mathcal{R}_{s}^{1}(G, \mathbb{k})$.

Proof. - For the first part, let $\rho: Q \rightarrow \mathbb{k}^{*}$ be a character. The 5 -term exact sequence associated to the extension $1 \rightarrow K \rightarrow G \rightarrow Q \rightarrow 1$ and the $\mathbb{k} G$-module $M=\mathbb{k}_{\rho \circ \varphi}$ ends in $H_{1}(G, M) \rightarrow H_{1}\left(Q, M_{K}\right) \rightarrow 0$, where $M_{K}=M /\{g m-m \mid m \in M, g \in K\}$ is the module of coinvariants under the action of $K$. Clearly, $M_{K}$ is isomorphic as 
a $\mathbb{k} Q$-module to $\mathbb{k}_{\rho}$. Hence, $\operatorname{dim}_{\mathbb{k}} H_{1}\left(G, \mathbb{k}_{\rho \circ \varphi}\right)$ is bounded below by $\operatorname{dim}_{\mathbb{k}} H_{1}\left(Q, \mathbb{k}_{\rho}\right)$. Thus, if $\rho \in \mathcal{V}_{s}^{1}(Q, \mathbb{k})$, then $\hat{\varphi}(\rho) \in \mathcal{V}_{s}^{1}(G, \mathbb{k})$, and we are done.

For the second part, consider the commuting diagram

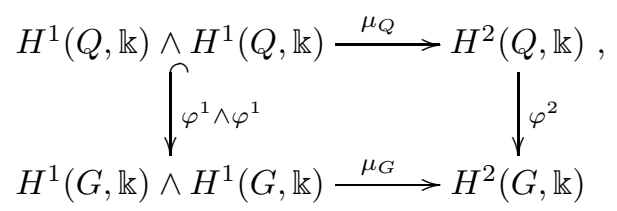

where $\mu_{Q}$ and $\mu_{G}$ are the respective cup-product maps. Let $a$ be a nonzero element in $A^{1}=H^{1}(Q, \mathbb{k})$. By definition, $a$ belongs to $\mathcal{R}_{s}^{1}(Q, \mathbb{k})$ if there exist linearly independent elements $b_{1}, \ldots, b_{s} \in A^{1}$ such that $a \wedge b_{i} \neq 0$ in $A^{1} \wedge A^{1}$, yet $\mu_{Q}\left(a, b_{i}\right)=0$. Clearly, $\varphi^{1}\left(b_{1}\right), \ldots, \varphi^{1}\left(b_{s}\right)$ are linearly independent in $H^{1}(G, \mathbb{k})$ and $\varphi^{1}(a) \wedge \varphi^{1}\left(b_{i}\right) \neq 0$, by injectivity of $\varphi^{1}$. Moreover, $\mu_{G}\left(\varphi^{1}(a) \wedge \varphi^{1}\left(b_{i}\right)\right)=0$, by commutativity of the diagram. Hence, $\varphi^{1}(a)$ belongs to $\mathcal{R}_{s}^{1}(G, \mathbb{k})$.

A.5. Alexander polynomial. - As before, let $G=\left\langle x_{1}, \ldots, x_{n} \mid r_{1}, \ldots, r_{m}\right\rangle$ be a finitely presented group. For simplicity, we will assume $G_{\mathrm{ab}}$ is torsion-free (otherwise, we need to mod out its torsion subgroup), and will fix the coefficient field $\mathbb{k}=\mathbb{C}$.

The Alexander polynomial of the group $G$, denoted $\Delta_{G}$, is the greatest common divisor of the minors of size $n-1$ of the Alexander matrix $\Phi_{G}$. As such, it is an element in the ring of Laurent polynomials $\mathbb{C} G_{\mathrm{ab}}$, well-defined up to units.

The Alexander polynomial defines a hypersurface, $V\left(\Delta_{G}\right)$, in the complex algebraic torus $\widehat{G}$. As shown in [20, Corollary 3.2], this hypersurface can be recovered from the characteristic variety $\mathcal{V}_{1}(G)=\mathcal{V}_{1}^{1}(G, \mathbb{C})$. More precisely, either $\Delta_{G}=0$, or

$$
\check{\mathcal{V}}_{1}(G) \backslash\{1\}=V\left(\Delta_{G}\right) \backslash\{1\}
$$

where $\check{\mathcal{V}}_{1}(G)$ denotes the union of all codimension-one irreducible components of $\mathcal{V}_{1}(G)$. If $G$ is a 3 -manifold group, more can be said.

Proposition A.2 ([20]). — Let $M$ be a compact, connected, orientable 3-manifold without boundary. Let $G=\pi_{1}(M)$, and suppose $G_{\mathrm{ab}}$ is torsion-free. Then

$$
\mathcal{V}_{1}(G) \backslash\{1\}=V\left(\Delta_{G}\right) \backslash\{1\}
$$

In other words, at least away from the origin, the characteristic variety $\mathcal{V}_{1}(M)$ is the hypersurface defined by the Alexander polynomial $\Delta_{M}=\Delta_{G}$.

\section{B}

\section{Finite, regular abelian covers}

B.1. Regular covers. - As before, let $X$ be a connected CW-complex with finite 1-skeleton, and basepoint at the unique 0-cell, $x_{0}$. Consider a covering map, $p: Y \rightarrow$ $X$, with connected total space $Y$. The cell structure on $X$ lifts to a cell structure on $Y$, in such a way that $p$ is a cellular map. Fix a basepoint $y_{0} \in p^{-1}\left(x_{0}\right)$; the induced homomorphism, $p_{\sharp}: \pi_{1}\left(Y, y_{0}\right) \rightarrow \pi_{1}\left(X, x_{0}\right)$, is injective. The assignment 
$p \rightsquigarrow \operatorname{im}\left(p_{\sharp}\right)$, then, establishes a one-to-one to correspondence between basepointpreserving equivalence classes of connected covers of $X$ and subgroups of $\pi_{1}\left(X, x_{0}\right)$.

In this context, a special role is played by the regular covers of our space $X$, that is, those covers $p:\left(Y, y_{0}\right) \rightarrow\left(X, x_{0}\right)$ for which $\operatorname{im}\left(p_{\sharp}\right)$ is a normal subgroup of $G=\pi_{1}\left(X, x_{0}\right)$. For such a cover, let $A=G / \operatorname{im}\left(p_{\sharp}\right)$ be the quotient group, and let $\chi: G \rightarrow A$ be the canonical projection. We say that $A$ is the group of deck transformations, and $\chi$ is a classifying homomorphism for the cover $p$. Conversely, if $\chi: G \rightarrow A$ is an epimorphism to a (necessarily finitely generated) group $A$, there is a regular cover $X^{\chi} \rightarrow X$, whose classifying homomorphism is $\chi$.

Now let $f: X^{\prime} \rightarrow X$ be a map, and let $Y^{\prime} \rightarrow X^{\prime}$ be the cover obtained by pulling back the cover $X^{\chi} \rightarrow X$ along $f$. Then $Y^{\prime} \rightarrow X^{\prime}$ is a regular $A$-cover, classified by the homomorphism $\chi^{\prime}=\chi \circ f_{\sharp}$.

B.2. Homology of finite abelian covers. - The next theorem records a formula for the homology groups $H_{q}\left(X^{\chi}, \mathbb{k}\right)$, in the case when the group of deck-transformations $A$ is finite, and $\mathbb{k}$ is an algebraically closed field of characteristic not dividing the order of $A$. In the case $q=1$, this formula is well-known, and due to Libgober [34], Sakuma [57] and E. Hironaka [29] for $\mathbb{k}=\mathbb{C}$, and to Matei-Suciu [44] for other fields $\mathbb{k}$. We provide a self-contained proof, following [14, Theorem 2.5].

Let $\chi: G \rightarrow A$ be an epimorphism to a finite abelian group $A$, and let $\widehat{\chi}: \widehat{A} \rightarrow \widehat{G}$ be the induced morphism between character groups. Then $\hat{\chi}$ is injective, and its image is (non-canonically) isomorphic to $A$. Furthermore, if $\rho: G \rightarrow \mathbb{k}^{*}$ is a character belonging to $\operatorname{im}(\widehat{\chi})$, there is a unique character $\iota_{\rho}: A \rightarrow \mathbb{k}^{*}$ such that $\rho=\iota_{\rho} \circ \chi$.

Theorem B.1. - Let $X^{\chi} \rightarrow X$ be the regular cover defined by an epimorphism $\chi$ from $G=\pi_{1}\left(X, x_{0}\right)$ to a finite abelian group $A$. Let $\mathbb{k}$ be an algebraically closed field of characteristic not dividing the order of $A$. Then, for each $q \geq 0$,

$$
\operatorname{dim}_{\mathbb{k}} H_{q}\left(X^{\chi}, \mathbb{k}\right)=\sum_{s \geq 1}\left|\operatorname{im}(\widehat{\chi}) \cap \mathcal{V}_{s}^{q}(X, \mathbb{k})\right| .
$$

Proof. - The epimorphism $\chi$ puts a left $\mathbb{k}[G]$-module structure on the group algebra $\mathbb{k}[A]$. By Shapiro's Lemma, $H_{q}\left(X^{\chi}, \mathbb{k}\right)$ is isomorphic, as a right $\mathbb{k}[A]$-module, to $H_{q}(X, \mathbb{k}[A])$. By our assumption on $\mathbb{k}$, the algebra $\mathbb{k}[A]$ is completely reducible, with one-dimensional, irreducible representations parametrized by $\widehat{A}$. As a left $\mathbb{k}[G]$ module, each such representation is isomorphic to the image of $\widehat{\chi}: \widehat{A} \rightarrow \widehat{G}$. Thus,

$$
H_{q}(X, \mathbb{k}[A]) \cong \bigoplus_{\rho \in \operatorname{im}(\widehat{\chi})} H_{q}\left(X, \mathbb{k}_{\rho}\right) .
$$

By the remark above, $H_{q}\left(X, \mathbb{k}_{\rho}\right)$ is isomorphic, as a $\mathbb{k}[A]$-module, to $\left(\mathbb{k}_{\iota_{\rho}}\right)^{\oplus b}$ where $b=\operatorname{dim}_{\mathbb{k}} H_{q}\left(X, \mathbb{k}_{\rho}\right)$. By definition, $\rho \in \mathcal{V}_{s}^{q}(X, \mathbb{k})$ if and only if $\operatorname{dim}_{\mathbb{k}} H_{q}\left(X, \mathbb{k}_{\rho}\right) \geq s$. Putting things together, we obtain an isomorphism of $\mathbb{k}[A]$-modules,

$$
H_{q}\left(X^{\chi}, \mathbb{k}\right) \cong \bigoplus_{s \geq 1} \bigoplus_{\rho \in \operatorname{im}(\widehat{\chi}) \cap \mathcal{V}_{s}^{q}(X, \mathbb{k})} \mathbb{k}_{\iota_{\rho}} .
$$

Taking dimensions on both sides completes the proof. 
B.3. The characteristic polynomial of the algebraic monodromy. - We now specialize to the case when $X^{\chi} \rightarrow X$ is a regular cover, classified by an epimorphism $\chi: \pi_{1}\left(X, x_{0}\right) \rightarrow A$ to a finite cyclic group $A$. Choose a generator $\alpha$ of $A$. Let $h=h_{\alpha}: X^{\chi} \rightarrow X^{\chi}$ be the corresponding monodromy automorphism, and let $h_{*}: H_{q}\left(X^{\chi}, \mathbb{k}\right) \rightarrow H_{q}\left(X^{\chi}, \mathbb{k}\right)$ be the induced map in homology. As in [14, §2.4], then, we obtain the following application of Theorem B.1.

Theorem B.2. - Assume char $(\mathbb{k}) \nmid|A|$. Then, the characteristic polynomial of the algebraic monodromy, $\Delta_{\chi, q}^{\mathbb{k}}(t)=\operatorname{det}\left(t \cdot \mathrm{id}-h_{*}\right)$, is given by

$$
\Delta_{\chi, q}^{\mathrm{k}}(t)=\prod_{s \geq 1} \prod_{\rho \in \operatorname{im}(\widehat{\chi}) \cap \mathcal{V}_{s}^{q}(X, \mathbb{k})}\left(t-\iota_{\rho}(\alpha)\right) .
$$

Proof. - From the hypothesis, the $\mathbb{k}[A]$-module $H_{q}\left(X^{\chi}, \mathbb{k}\right)=H_{q}(X, \mathbb{k}[A])$ is completely reducible. Therefore, the automorphism $h_{*}$ is diagonalizable. Furthermore, the eigenvalues of $h_{*}$, counted with multiplicity, are indexed by the irreducible $\mathbb{k}[A]$ modules appearing in decomposition (76), and we are done.

In degree $q=1$, and for $\mathbb{k}=\mathbb{C}$, the polynomial $\Delta_{\chi}(t)=\Delta_{\chi, 1}^{\mathbb{C}}(t)$ can be related to the Alexander polynomial of the group $G=\pi_{1}(X)$, as follows:

$$
\Delta_{\chi}(t)=(t-1)^{c} \Delta_{G}\left(t^{\chi_{1}}, \ldots, t^{\chi_{n}}\right),
$$

where $c$ is an integer depending only on $G$.

B.4. Jump loci of finite covers. - The next proposition and its corollary were proved in [19] in the case when $\mathbb{k}$ has characteristic 0 . For completeness, we include a proof, valid in a slightly more general context.

Proposition B.3. - Let $A$ be a finite group, and let $p: Y \rightarrow X$ be a regular $A$ cover. Suppose that $\operatorname{char}(\mathbb{k}) \nmid|A|$, and $\operatorname{char}(\mathbb{k}) \neq 2$ if $H_{1}(X, \mathbb{Z})$ has 2-torsion. Then $p^{*}\left(\mathcal{V}_{s}^{q}(X, \mathbb{k})\right) \subseteq \mathcal{V}_{s}^{q}(Y, \mathbb{k})$ and $p^{*}\left(\mathcal{R}_{s}^{q}(X, \mathbb{k})\right) \subseteq \mathcal{R}_{s}^{q}(Y, \mathbb{k})$, for all $q \geq 0$ and $s \geq 1$.

Proof. - For the first claim, let $\mathcal{L}$ be a $\mathbb{k}$-valued, rank-1 local system on $X$, and consider the Hochschild-Serre spectral sequence of the cover, $E_{i j}^{2}=H_{i}\left(A, H_{j}\left(Y, p^{*} \mathcal{L}\right)\right) \Rightarrow$ $H_{i+j}(X, \mathcal{L})$. Since $A$ is finite and $\operatorname{char}(\mathbb{k}) \nmid|A|$, we have that $E_{i j}^{2}=0$, for all $i>0$ and $j \geq 0$; thus, the spectral sequence collapses to an isomorphism, $H_{*}(X, \mathcal{L}) \cong$ $H_{*}\left(Y, p^{*} \mathcal{L}\right)_{A}$. By duality, we obtain an injection, $p^{*}: H^{*}(X, \mathcal{L}) \hookrightarrow H^{*}\left(Y, p^{*} \mathcal{L}\right)$, and we are done.

For the second claim, a standard transfer argument, using again the hypothesis on char $(\mathbb{k})$, allows us to identify the induced algebra map in cohomology, $p^{*}: H^{*}(X, \mathbb{k}) \rightarrow$ $H^{*}(Y, \mathbb{k})$, with the inclusion $p^{*}: H^{*}(Y, \mathbb{k})^{A} \hookrightarrow H^{*}(Y, \mathbb{k})$. For a class $a \in H^{1}(Y, \mathbb{k})^{A}$, the monodromy action of $A$ on $H^{*}(Y, \mathbb{k})$ gives rise to an action on the chain complex $\left(H^{*}(Y, \mathbb{k}), \cdot a\right)$, with fixed subcomplex $\left(H^{*}(X, \mathbb{k}), \cdot a\right)$. We thus obtain an inclusion $H^{*}\left(H^{*}(X, \mathbb{k}), \cdot a\right) \hookrightarrow H^{*}\left(H^{*}(Y, \mathbb{k}), \cdot a\right)$, and we are done.

The proof of the second part of the proposition has an immediate corollary.

Corollary B.4. - With notation as above, suppose $A$ acts trivially on $H^{1}(Y, \mathbb{k})$. Then $p^{*}: \mathcal{R}_{s}^{1}(X, \mathbb{k}) \rightarrow \mathcal{R}_{s}^{1}(Y, \mathbb{k})$ is an isomorphism, for all $s \geq 1$. 
The assumption of the corollary is really necessary. For instance, if $X$ is a wedge of $n>1$ circles, then $Y$ is a wedge of $m=(n-1)|A|+1$ circles; thus, if $|A|>1$, then the map $p^{*}: \mathcal{R}_{1}^{1}(X, \mathbb{k}) \rightarrow \mathcal{R}_{1}^{1}(Y, \mathbb{k})$ is a proper inclusion, sending $\mathbb{k}^{n}$ into $\mathbb{k}^{m}$.

\section{C}

\section{Formality}

C.1. Formal spaces. - Let $X$ be connected CW-complex with finite 1-skeleton. To such a space, Sullivan associated the commutative differential graded algebra (cdga) of polynomial differential forms on $X$ with coefficients in $\mathbb{Q}$, denoted $A_{\mathrm{PL}}(X, \mathbb{Q})$.

Let $H^{*}(X, \mathbb{Q})$ be the rational cohomology algebra of $X$, endowed with the zero differential. The space $X$ is said to be formal if there is a zig-zag of cdga morphisms connecting $A_{\mathrm{PL}}(X, \mathbb{Q})$ to $H^{*}(X, \mathbb{Q})$, with each such morphism inducing an isomorphism in cohomology. The space $X$ is merely $k$-formal (for some $k \geq 1$ ) if each of these morphisms induces an isomorphism in degrees up to $k$, and a monomorphism in degree $k+1$. If $X$ is a $k$-formal and $\operatorname{dim} X \leq k+1$, then $X$ is formal.

If $X$ is a smooth manifold, Sullivan's algebra may be replaced by the de Rham algebra $\Omega_{\mathrm{dR}}^{*}(X)$ of smooth, differential forms on $X$. Examples of formal spaces include suspensions, rational cohomology tori, surfaces, compact connected Lie groups, as well as their classifying spaces. On the other hand, the only nilmanifolds which are formal are tori. Formality is preserved under wedges and products of spaces, and connected sums of manifolds.

The 1-minimality property of a space $X$ depends only on its fundamental group, $G=\pi_{1}\left(X, x_{0}\right)$. Alternatively, a finitely generated group $G$ is 1 -formal if and only if its Malcev Lie algebra (defined as the Lie algebra of primitive elements in the $I$-adic completion of the group-algebra $\mathbb{Q}[G]$ ) admits a quadratic presentation. Examples of 1-formal groups include free groups and free abelian groups of finite rank, surface groups, and groups with first Betti number equal to 0 or 1 . The 1-formality property is preserved under free products and direct products.

A classical obstruction to formality is provided by the higher-order Massey products. In particular, if $X$ is 1-formal, and $\alpha_{1}, \alpha_{2}, \alpha_{3}$ are elements in $H^{1}(X, \mathbb{Q})$ such that $\alpha_{1} \alpha_{2}=\alpha_{2} \alpha_{3}=0$, then the Massey triple product $\left\langle\alpha_{1}, \alpha_{2}, \alpha_{3}\right\rangle$ must vanish, modulo indeterminacy.

One can make an analogous definition of $\mathbb{k}$-formality over an arbitrary field $\mathbb{k}$. Again, an obstruction for $\mathbb{k}$-formality is provided by the Massey products in $H^{*}(X, \mathbb{k})$.

C.2. Formality in finite covers. - Suppose $f: X \rightarrow Y$ is a map such that $f^{*}: H^{*}(Y, \mathbb{Q}) \rightarrow H^{*}(X, \mathbb{Q})$ is an isomorphism up to degree $k$, and a monomorphism in degree $k+1$. Then $X$ is $k$-formal if and only if $Y$ is $k$-formal.

Lemma C.1. - Let $p: Y \rightarrow X$ be a finite, regular cover. If $Y$ is $k$-formal, then $X$ is also $k$-formal.

Proof. - Let $\Gamma$ be the group of deck-transformations, so that $X=Y / \Gamma$. As shown in [26, Remark 3.30(2)], if $Y$ is formal, then $X$ is also formal. The same argument applies to $k$-formality. 
A particular case is worth mentioning. Suppose $G$ is a finitely generated group, and $H \triangleleft G$ is a finite-index, normal subgroup. If $H$ is 1-formal, then $G$ is also 1-formal. As the next example shows, the converse does not hold.

Example C.2. - As is well-known, the Heisenberg group $H=\langle x, y|[x, y]$ central $\rangle$ admits non-trivial triple Massey products in $H^{1}(H, \mathbb{Q})$; thus, $H$ is not 1 -formal. On the other hand, the semi-direct product $G=H \rtimes \mathbb{Z}_{2}$ defined by the involution $x \mapsto$ $x^{-1}, y \mapsto y^{-1}$ has $b_{1}(G)=0$, and so $G$ is 1 -formal.

Nevertheless, the converse to Lemma C.1 holds under an additional, rather restrictive condition.

Lemma C.3 ([19]). - Let $p: Y \rightarrow X$ be a finite, regular cover, and suppose the group of deck-transformations acts trivially on $H^{i}(Y, \mathbb{Q})$, for all $i \leq k$. Then $Y$ is $k$-formal if and only if $X$ is $k$-formal.

C.3. The tangent cone formula. - Let $G=\pi_{1}\left(X, x_{0}\right)$. The homomorphism $\mathbb{C} \rightarrow \mathbb{C}^{*}, z \mapsto e^{z}$ induces a homomorphism $\exp : \operatorname{Hom}(G, \mathbb{C}) \rightarrow \operatorname{Hom}\left(G, \mathbb{C}^{*}\right)=\widehat{G}$. Given a subvariety of $W \subseteq \widehat{G}$, define its exponential tangent cone at the origin to be the set

$$
\tau_{1}(W)=\{z \in \operatorname{Hom}(G, \mathbb{C}) \mid \exp (\lambda z) \in W, \text { for all } \lambda \in \mathbb{C}\} .
$$

It turns out that $\tau_{1}(W)$ is a finite union of rationally defined linear subspaces (see [21], and also [62] for more details). Moreover, $\tau_{1}(W)$ is included in $\operatorname{TC}_{1}(W)$, the usual tangent cone to $W$ at the origin.

Now fix an integer $s>0$, and consider the varieties $\mathcal{V}_{s}^{1}(X) \subseteq \widehat{G}$ and $\mathcal{R}_{s}^{1}(X) \subseteq$ $H^{1}(X, \mathbb{C})=\operatorname{Hom}(G, \mathbb{C})$. By work of Libgober [36], the tangent cone to $\mathcal{V}_{s}^{1}(X)$ at 1 is included in $\mathcal{R}_{s}^{1}(X)$. Thus, we get a chain of inclusions,

$$
\tau_{1}\left(\mathcal{V}_{s}^{1}(X)\right) \subseteq \operatorname{TC}_{1}\left(\mathcal{V}_{s}^{1}(X)\right) \subseteq \mathcal{R}_{s}^{1}(X),
$$

each of which is a proper inclusion, in general. The main connection between the formality property of a space and its cohomology jump loci is provided by the following theorem.

Theorem C.4 ([21]). - Let X be a 1-formal space. For each $s>0$, the following "tangent cone formula" holds:

$$
\tau_{1}\left(\mathcal{V}_{s}^{1}(X)\right)=\operatorname{TC}_{1}\left(\mathcal{V}_{s}^{1}(X)\right)=\mathcal{R}_{s}^{1}(X) .
$$

As a consequence, the irreducible components of $\mathcal{R}_{s}^{1}(X)$ are all rationally defined subspaces, while the components of $\mathcal{V}_{s}^{1}(X)$ passing through the origin are all algebraic subtori of the form $\exp (L)$, with $L$ running through the irreducible components of $\mathcal{R}_{s}^{1}(X)$. 


\section{References}

[1] D. Arapura, Geometry of cohomology support loci for local systems. I., J. Algebraic Geom. 6 (1997), no. 3, 563-597. MR1487227 (98m:32043) 3.3, 3.8, 5.5

[2] E. Artal Bartolo, J. Cogolludo, D. Matei, Characteristic varieties of quasi-projective manifolds and orbifolds, Geom. Topol. 17 (2013), no. 1, 273-309. MR3035328 3.3, 3.4, 3.8

[3] E. Brieskorn, Sur les groupes de tresses (d'après V. I. Arnol'd), Séminaire Bourbaki, 24ème année (1971/1972), Exp. No. 401, pp. 21-44, Lecture Notes in Math., vol. 317, Springer, Berlin, 1973. MR0422674 (54 \#10660) 1.2, 2.3, 2.3

[4] N. Budur, A. Dimca, M. Saito, First Milnor cohomology of hyperplane arrangements, in: Topology of algebraic varieties and singularities, 279-292, Contemp. Math., vol. 538, Amer. Math. Soc., Providence, RI, 2011. MR2777825 (2012f:32040) 5.2

[5] N. Budur, B. Wang, Cohomology jump loci of quasi-projective varieties, arXiv: 1211.3766v1. 3.3, 5.5

[6] D. Cohen, G. Denham, A. Suciu, Torsion in Milnor fiber homology, Alg. Geom. Topology 3 (2003), 511-535. MR1997327 (2004d:32043) 1.3, 4.6, 5.3, 5.3

[7] D. Cohen, A. Dimca, P. Orlik, Nonresonance conditions for arrangements, Annales Institut Fourier (Grenoble) 53 (2003), no. 6, 1883-1896. MR2038782 (2005a:32027) 5.7

[8] D. Cohen, A. Suciu, On Milnor fibrations of arrangements, J. London Math. Soc. (2) 51 (1995), no. 1, 105-119. MR1310725 (96e:32034) 1.3, 4.6, 5.3, 5.2, 5.11

[9] D. Cohen, A. Suciu, The braid monodromy of plane algebraic curves and hyperplane arrangements, Comment. Math. Helvetici 72 (1997), no. 2, 285-315. MR1470093 (98f:52012) $3.1,3.1$

[10] D. Cohen, A. Suciu, Characteristic varieties of arrangements, Math. Proc. Cambridge Phil. Soc. 127 (1999), no. 1, 33-53. MR1692519 (2000m:32036) 2.4

[11] D. Cohen, A. Suciu, Boundary manifolds of projective hypersurfaces, Advances in Math. 206 (2006), no. 2, 538-566 MR2263714 (2007j:14064) 1.4, 6, 6.1, 6.2, 6.4, 6.3, 6.3, 7.3

[12] D. Cohen, A. Suciu, The boundary manifold of a complex line arrangement, Geometry \& Topology Monographs 13 (2008), 105-146. MR2508203 (2010c:32051) 1.4, 6, 6.3, 6.6, $6.9,6.7,6.11$

[13] G. Denham, Homological aspects of hyperplane arrangements, in: Arrangements, local systems and singularities, 39-58, Progress in Math., vol. 283, Birkhäuser, Basel, 2010. MR3025859 3.2

[14] G. Denham, A. Suciu, Multinets, parallel connections, and Milnor fibrations of arrangements, Proc. London Math. Soc. (first published online December 11, 2013), doi:10.1112/plms/pdt058, available at arXiv:1209.3414v2. 1.3, 3.3, 3.6, 3.10, 4.6, 5.3, $5.13,5.3,5.3,5.4,5.17$, B.2, B.3

[15] A. Dimca, Singularities and topology of hypersurfaces, Universitext, Springer-Verlag, New York, 1992. MR1194180 (94b:32058) 6.1, 7.1, 7.3

[16] A. Dimca, Sheaves in topology, Universitext, Springer-Verlag, Berlin, 2004. MR2050072 (2005j:55002) 5.7

[17] A. Dimca, Characteristic varieties and constructible sheaves, Rend. Lincei Mat. Appl. 18 (2007), no. 4, 365-389. MR2349994 (2009a:14006) 3.3, 3.4, 3.8

[18] A. Dimca, Monodromy of triple point line arrangements, arXiv:1107.2214v2. 5.2

[19] A. Dimca, S. Papadima, Finite Galois covers, cohomology jump loci, formality properties, and multinets, Ann. Sc. Norm. Super. Pisa Cl. Sci. 10 (2011), no. 2, 253-268. MR2856148 (2012i:32033) 1.3, 5.5, 5.19, 5.20, B.4, C.3 
[20] A. Dimca, S. Papadima, A. Suciu, Alexander polynomials: Essential variables and multiplicities, Int. Math. Res. Notices 2008, no. 3, Art. ID rnm119, 36 pp. MR2416998 (2009i:32036) A.5, A.2

[21] A. Dimca, S. Papadima, A. Suciu, Topology and geometry of cohomology jump loci, Duke Math. Journal 148 (2009), no. 3, 405-457. MR2527322 (2011b:14047) C.3, C.4

[22] A. Durfee, Neighborhoods of algebraic sets, Trans. Amer. Math. Soc. 276 (1983), no. 2, 517-530; MR0688959 (84m:32013) 6.1

[23] M. Falk, Arrangements and cohomology, Ann. Combin. 1 (1997), no. 2, 135-157. MR1629681 (99g:52017) 2.4

[24] M. Falk, Resonance varieties over fields of positive characteristic, Int. Math. Research Notices 2007 (2007), no. 3, article ID rnm009, 25 pages. MR2337033 (2008m:14107) 1.2, $2.8,2.17,2.18$

[25] M. Falk, S. Yuzvinsky, Multinets, resonance varieties, and pencils of plane curves, Compositio Math. 143 (2007), no. 4, 1069-1088. MR2339840 (2009e:52043) 1.2, 2.5, 2.5, 2.6, $2.6,2.11,3.5,3.5$

[26] Y. Félix, J. Oprea, D. Tanré, Algebraic models in geometry, Oxford Grad. Texts in Math., vol. 17, Oxford Univ. Press, Oxford, 2008. MR2403898 (2009a:55006) C.2

[27] A. Hatcher, Algebraic topology, Cambridge University Press, Cambridge, 2002. MR1867354 (2002k:55001) 4.5

[28] E. Hironaka, Abelian coverings of the complex projective plane branched along configurations of real lines, Memoirs A.M.S., vol. 502, Amer. Math. Soc., Providence, RI, 1993. MR1164128 (94b:14020) 3.1

[29] E. Hironaka, Alexander stratifications of character varieties, Annales de l'Institut Fourier (Grenoble) 47 (1997), no. 2, 555-583. MR1450425 (98e:14020) A.3, B.2

[30] E. Hironaka, Boundary manifolds of line arrangements, Math. Annalen 319 (2001), no. 1, 17-32. MR1812817 (2001m:32061) 6.4

[31] F. Hirzebruch, The topology of normal singularities of an algebraic surface (after D. Mumford), Séminaire Bourbaki, Vol. 8, Exp. No. 250, 129-137, Soc. Math. France, Paris, 1995. MR1611536 6.5

[32] T. Jiang, S.S.-T. Yau, Topological invariance of intersection lattices of arrangements in $\mathbb{C P}^{2}$, Bull. Amer. Math. Soc. 29 (1993), no. 1, 88-93. MR1197426 (94b:52022) 6.4

[33] T. Jiang, S.S.-T. Yau, Intersection lattices and topological structures of complements of arrangements in $\mathbb{C P}^{2}$, Ann. Scuola Norm. Sup. Pisa Cl. Sci. (4) 26 (1998), no. 2, 357-381; MR1631597 (99g:52020) 6.4

[34] A. Libgober, On the homology of finite abelian coverings, Topology Appl. 43 (1992), no. 2, 157-166. MR1152316 (93e:57003) B.2

[35] A. Libgober, Characteristic varieties of algebraic curves, in: Applications of algebraic geometry to coding theory, physics and computation (Eilat, 2001), pp. 215-254, NATO Sci. Ser. II Math. Phys. Chem., vol. 36, Kluwer Acad. Publ., Dordrecht, 2001. MR1866902 (2003e:14008) 2.4

[36] A. Libgober, First order deformations for rank one local systems with a non-vanishing cohomology, Topology Appl. 118 (2002), no. 1-2, 159-168. MR1877722 (2002m:52025) C.3

[37] A. Libgober, Eigenvalues for the monodromy of the Milnor fibers of arrangements, Trends in singularities, pp. 141-150, Trends Math., Birkhäuser, Basel, 2002. MR1900784 (2003f:32035) 5.3

[38] A. Libgober, Non vanishing loci of Hodge numbers of local systems, Manuscripta Math. 128 (2009), no. 1, 1-31. MR2470184 (2010g:32026) 3.3 
[39] A. Libgober, On combinatorial invariance of the cohomology of Milnor fiber of arrangements and Catalan equation over function field, in: Arrangements of hyperplanes (Sapporo 2009), 175-187, Adv. Stud. Pure Math., vol. 62, Math. Soc. Japan, Tokyo, 2012. MR2933797 5.2

[40] A. Libgober, S. Yuzvinsky, Cohomology of Orlik-Solomon algebras and local systems, Compositio Math. 21 (2000), no. 3, 337-361. MR1761630 (2001j:52032) 1.2, 2.4

[41] A. Măcinic, S. Papadima, On the monodromy action on Milnor fibers of graphic arrangements, Topology Appl. 156 (2009), no. 4, 761-774. MR2492960 (2010i:32028) 5.3, 5.2

[42] D. Matei, Massey products of complex hypersurface complements, In: Singularity Theory and its Applications, 205-219, Adv. Studies in Pure Math., vol. 43, Math. Soc. Japan, Tokyo, 2007. MR2325139 (2008h:32041) 1.2, 2.19

[43] D. Matei, A. Suciu, Cohomology rings and nilpotent quotients of real and complex arrangements, in: Arrangements-Tokyo 1998, pp. 185-215, Adv. Stud. Pure Math., vol. 27, Math. Soc. Japan, Tokyo, 2000. MR1796900 (2002b:32045) 1.2, 2.8, 2.16, A.3

[44] D. Matei, A. Suciu, Hall invariants, homology of subgroups, and characteristic varieties, Internat. Math. Res. Notices 2002 (2002), no. 9, 465-503. MR1884468 (2003d:20055) B.2

[45] J. Milnor, Singular points of complex hypersurfaces, Annals of Math. Studies, vol. 61, Princeton Univ. Press, Princeton, NJ, 1968. MR0239612 (39 \#969) 1.3, 4.1, 7.2

[46] A. Némethi, A. Szilárd, Milnor fiber boundary of a non-isolated surface singularity, Lecture Notes in Math, vol. 2037, Springer-Verlag, Berlin Heidelberg, 2012. MR3024944 $1.4,7.4,7.7,7.5,7.8,7.7,7.7,7.7$

[47] P. Orlik, R. Randell, The Milnor fiber of a generic arrangement, Arkiv für Mat. 31 (1993), no. 1, 71-81. MR1230266 (95e:32044) 4.6

[48] P. Orlik, L. Solomon, Combinatorics and topology of complements of hyperplanes, Invent. Math. 56 (1980), no. 2, 167-189; MR0558866 (81e:32015) 1.2, 2.3

[49] P. Orlik, H. Terao, Arrangements of hyperplanes, Grundlehren Math. Wiss., vol. 300, Springer-Verlag, Berlin, 1992; MR1217488 (94e:52014) 1.2, 2.3

[50] J. Oxley, Matroid theory, Oxford Sci. Publ, Oxford University Press, New York, 1992. MR1207587 (94d:05033) 2.14, 2.15

[51] S. Papadima, A. Suciu, Chen Lie algebras, Intern. Math. Res. Notices 2004 (2004), no. 21, 1057-1086. MR2037049 (2004m:17043) 3.2

[52] S. Papadima, A. Suciu, Algebraic invariants for right-angled Artin groups, Math. Ann. 334 (2006), no. 3, 533-555. MR2207874 (2006k:20078) A.4

[53] S. Papadima, A. Suciu, Geometric and algebraic aspects of 1-formality, Bull. Math. Soc. Sci. Math. Roumanie 52 (2009), no. 3, 355-375. MR2554494 (2010k:55018) 5.6

[54] S. Papadima, A. Suciu, Bieri-Neumann-Strebel-Renz invariants and homology jumping loci, Proc. London Math. Soc. 100 (2010), no. 3, 795-834. MR2640291 (2011i:55006) A.1, A.2, A.3

[55] J. Pereira, S. Yuzvinsky, Completely reducible hypersurfaces in a pencil, Adv. Math. 219 (2008), no. 2, 672-688. MR2435653 (2009g:14010) 2.5

[56] G. Rybnikov, On the fundamental group of the complement of a complex hyperplane arrangement, Funct. Anal. Appl. 45 (2011), no. 2, 137-148 MR2848779 (2012i:14067) 3.1

[57] M. Sakuma, Homology of abelian coverings of links and spatial graphs, Canad. J. Math. 47 (1995), no. 1, 201-224. MR1319696 (96d:57008) B.2

[58] A. Suciu, Fundamental groups of line arrangements: Enumerative aspects, in: Advances in algebraic geometry motivated by physics (Lowell, MA, 2000), pp. 43-79, Contemp. Math., vol 276, Amer. Math. Soc., Providence, RI, 2001. MR1837109 (2002k:14029) 1.2, 2.16, 3.2 
[59] A. Suciu, Translated tori in the characteristic varieties of complex hyperplane arrangements, Topology Appl. 118 (2002), no. 1-2, 209-223. MR1877726 (2002j:32027) 3.11, 3.6

[60] A. Suciu, Fundamental groups, Alexander invariants, and cohomology jumping loci, in: Topology of algebraic varieties and singularities, 179-223, Contemp. Math., vol. 538, Amer. Math. Soc., Providence, RI, 2011. MR2777821 (2012b:20092) 4.6

[61] A. Suciu, Geometric and homological finiteness in free abelian covers, in: Configuration Spaces: Geometry, Combinatorics and Topology (Centro De Giorgi, 2010), 461-501, Publications of the Scuola Normale Superiore, vol. 14, Edizioni della Normale, Pisa, 2012, available at arXiv: $1112.0948 v 2.4 .9$

[62] A. Suciu, Characteristic varieties and Betti numbers of free abelian covers, Intern. Math. Res. Notices (first published online November 16, 2012), doi:10.1093/imrn/rns246, available at arXiv:1111.5803v3. 4.9, A.4, C.3

[63] A. Suciu, Y. Yang, G. Zhao, Homological finiteness of abelian covers, Ann. Sc. Norm. Super. Pisa Cl. Sci. (to appear), available at arXiv: 1204.4873v2. 4.9

[64] E. Westlund, The boundary manifold of an arrangement, Ph.D. thesis, University of Wisconsin, Madison, WI, 1997 MR2697010 6.4, 6.5

[65] M. Yoshinaga, Milnor fibers of real line arrangements, J. Singul. 7 (2013), 242-259. MR3090727 5.2

[66] S. Yuzvinsky, A new bound on the number of special fibers in a pencil of curves, Proc. Amer. Math. Soc. 137 (2009), no. 5, 1641-1648. MR2470822 (2009k:14015) 2.5

[67] S. Yuzvinsky, Resonance varieties of arrangement complements, in: Arrangements of Hyperplanes (Sapporo 2009), 553-570, Advanced Studies Pure Math., vol. 62, Kinokuniya, Tokyo, 2012. MR2933810 2.5

[68] H. Zuber, Non-formality of Milnor fibers of line arrangements, Bull. London Math. Soc. 42 (2010), no. 5, 905-911. MR2728693 (2012b:32041) 1.3, 5.6, 5.22

Alexander I. Suciu, Department of Mathematics, Northeastern University, Boston, MA 02115, USA • E-mail : a.suciu@neu.edu • Url : http://www.northeastern.edu/suciu/ 